\title{
WEIGHTED RESTRICTION ESTIMATES USING POLYNOMIAL PARTITIONING
}

\author{
BASSAM SHAYYA
}

\begin{abstract}
We use the polynomial partitioning method of Guth 8 to prove weighted Fourier restriction estimates in $\mathbb{R}^{3}$ with exponents $p$ that range between 3 and 3.25, depending on the weight. As a corollary to our main theorem, we obtain new (non-weighted) local and global restriction estimates for compact $C^{\infty}$ surfaces $S \subset \mathbb{R}^{3}$ with strictly positive second fundamental form. For example, we establish the global restriction estimate $\|E f\|_{L^{p}\left(\mathbb{R}^{3}\right)} \lesssim\|f\|_{L^{q}(S)}$ in the full conjectured range of exponents for $p>3.25$ (up to the sharp line), and the global restriction estimate $\|E f\|_{L^{p}(\Omega)} \lesssim\|f\|_{L^{2}(S)}$ for $p>3$ and certain sets $\Omega \subset \mathbb{R}^{3}$ of infinite Lebesgue measure. As a corollary to our main theorem, we also obtain new results on the decay of spherical means of Fourier transforms of positive compactly supported measures on $\mathbb{R}^{3}$ with finite $\alpha$ dimensional energies.
\end{abstract}

\section{INTRODUCTION}

There has been a surge of activity in recent years of using polynomial partitioning methods to study some important and open problems in combinatorics and harmonic analysis. One striking example is Guth's recent paper [8], where polynomial partitioning was used to obtain progress on the Fourier restriction problem in $\mathbb{R}^{3}$.

Let $S$ be a compact $C^{\infty}$ surface in $\mathbb{R}^{3}$ with strictly positive second fundamental form. In a recent paper [8, Guth used polynomial partitioning to obtain a new local restriction estimate on $S$ : to every $\epsilon>0$ there is a constant $C_{\epsilon}$ (which also depends on $S$ ) such that

$$
\int_{|x| \leq R}|E f(x)|^{3.25} d x \leq C_{\epsilon} R^{\epsilon}\|f\|_{L^{\infty}(S)}^{3.25}
$$

for all $f \in L^{\infty}(S)=L^{\infty}(\sigma)$, where $\sigma$ is the surface measure on $S$ and $E f$ is the Fourier extension (or adjoint restriction) operator on $S$ defined as

$$
E f(x)=E_{S} f(x)=\widehat{f d \sigma}(x)=\int e^{-2 \pi i x \cdot \xi} f(\xi) d \sigma(\xi) .
$$

Using Tao's $\epsilon$-removal lemma, (1) can be turned into a global restriction estimate:

$$
\int_{\mathbb{R}^{3}}|E f(x)|^{p} d x \leq C\|f\|_{L^{\infty}(S)}^{p} \quad(p>3.25)
$$

for all $f \in L^{\infty}(S)$.

The restriction conjecture in harmonic analysis asserts that the exponent 3.25 in (11) and (2) can be replaced by 3. Guth's theorem is the current best known result on this conjecture in $\mathbb{R}^{3}$. The purpose of this paper is to lower the 3.25 exponent by

2010 Mathematics Subject Classification. 42B10, 42B20; 28A75. 
considering weighted variants of (11). For example, as a consequence of the results of this paper, one obtains the estimates

$$
\int_{B_{R}}|E f(x)|^{3} \chi_{\Omega_{1}}(x) d x \leq C_{\epsilon} R^{\epsilon}\|f\|_{L^{2}(S)}^{3}
$$

for all $f \in L^{2}(S)$, and

$$
\int_{B_{R}}|E f(x)|^{22 / 7} \chi_{\Omega_{2}}(x) d x \leq C_{\epsilon} R^{\epsilon}\|f\|_{L^{2}(S)}^{3}\|f\|_{L^{\infty}(S)}^{1 / 7}
$$

for all $f \in L^{\infty}(S)$, where $B_{R}$ is any ball in $\mathbb{R}^{3}$ of radius $R, \chi_{\Omega_{1}}$ is the characteristic function of the set

$$
\Omega_{1}=\left\{\left(x_{1}, x_{2}, x_{3}\right) \in \mathbb{R}^{3}:\left|x_{3}\right| \leq\left|\left(x_{1}, x_{2}\right)\right|^{-1 / 2}\right\},
$$

and $\chi_{\Omega_{2}}$ is the characteristic function of the set

$$
\Omega_{2}=\left\{\left(x_{1}, x_{2}, x_{3}\right) \in \mathbb{R}^{3}:\left|x_{3}\right| \leq 1\right\} .
$$

We refer the reader to the paragraph following the statement of our main theorem, Theorem 1.1, for the proofs of (3) and (4).

Some of our local restriction estimates can be turned into global ones. For example, we establish the global version of (4):

$$
\int_{\mathbb{R}^{3}}|E f(x)|^{p} \chi_{\Omega_{2}}(x) d x \leq C\|f\|_{L^{44 / 21}(S)}^{p} \quad(p>22 / 7)
$$

for all $f \in L^{44 / 21}(S)$. For certain types of sets, we get global estimates for the full range of exponents $p>3$. For example, we prove that

$$
\int_{\mathbb{R}^{3}}|E f(x)|^{p} \chi_{\Omega}(x) d x \leq C\|f\|_{L^{2}(S)}^{p} \quad(p>3)
$$

for all $f \in L^{2}(S)$, where

$$
\Omega=\cup_{m=0}^{\infty} \cup_{n=0}^{\infty}\left(\mathbb{R} \times[-1,1]^{2}+\left(0, m^{4}, n^{4}\right)\right) .
$$

We also obtain the following improvement on (2):

$$
\int_{\mathbb{R}^{3}}|E f(x)|^{p} d x \leq C\|f\|_{L^{q}(S)}^{p} \quad\left(p>3.25, q^{\prime}<p / 2\right)
$$

for all $f \in L^{q}(S)$, where $q^{\prime}$ is the exponent conjugate to $q$. Up to the end point $q^{\prime}=p / 2$, the range of the $q$ exponent in (5) is known to be the best possible.

All the results of this paper concerning global Fourier restriction estimates are stated in Corollary 2.1.

Let $M\left(\mathbb{R}^{3}\right)$ be the space of all complex Borel measures on $\mathbb{R}^{3}$, and $S \subset \mathbb{R}^{3}$ be, as above, a compact $C^{\infty}$ surface with strictly positive second fundamental form. The second application of the main theorem of this paper concerns the decay properties of the $L^{q}$ norms

$$
\|\widehat{\mu}(R \cdot)\|_{L^{q}(S)}=\left(\int|\widehat{\mu}(R \xi)|^{q} d \sigma(\xi)\right)^{1 / q}
$$

as $R \rightarrow \infty$, where $\mu \in M\left(\mathbb{R}^{3}\right)$ is a positive compactly supported measure with finite $\alpha$-dimensional energy $I_{\alpha}(\mu)$ (see (6) for the definition of $I_{\alpha}(\mu)$ ). This is an important topic for the study of distance sets in geometric measure theory, as we explain in Section 3 below.

For $q=2$, Erdoğan [5] proved the decay estimate

$$
\|\widehat{\mu}(R \cdot)\|_{L^{2}\left(\mathbb{S}^{2}\right)} \leq C_{\epsilon} R^{\epsilon} R^{-(\alpha / 4)-(1 / 8)} \sqrt{I_{\alpha}(\mu)}
$$


for $3 / 2 \leq \alpha \leq 5 / 2$, where $\mathbb{S}^{2}$ is the unit sphere in $\mathbb{R}^{3}$ (see (8) for the $n$-dimensional version of this estimate), and used it to get the best known result on the distance set problem in $\mathbb{R}^{3}$, but no better decay estimate was known for $1 \leq q<2$. In this paper, we get a better decay estimate for the range of exponents $1 \leq q \leq p_{0}$, where $p_{0}=4(4 \alpha+3) /(10 \alpha+3)$ and $3 / 2<\alpha<5 / 2$. We also reprove Erdoğan's estimate when $3 / 2 \leq \alpha<2$, obtaining a proof of his distance set result that is based on polynomial partitioning. All these results are stated in Corollary 3.1 .

Let $\mu$ be a positive and compactly supported measure in $M\left(\mathbb{R}^{3}\right)$ satisfying

$$
\sup _{x \in \mathbb{R}^{3}} \sup _{r>0} \frac{\mu(B(x, r))}{r^{\alpha}}<\infty .
$$

(We refer the reader to the first paragraph of Section 4 for an explanation of how this condition relates to the condition $I_{\alpha}(\mu)<\infty$.) The third application of our main theorem establishes $L^{p}(\mu)$ bounds on exponential sums of the form $\sum_{l=1}^{N} a_{l} e^{2 \pi i R w_{l} \cdot x}$, where $w_{1}, \ldots, w_{N} \in S$ are $R^{-1}$-separated. These results are stated in Corollary 4.1.

1.1. The main theorem. Suppose $3 / 2 \leq \alpha \leq 3$ and $H$ is a non-negative measurable function on $\mathbb{R}^{3}$. We define $A_{\alpha}(H)$ to be the infimum of the set

$$
\left\{\lambda \in[0, \infty]: \int_{B\left(x_{0}, R\right)} H(x) d x \leq \lambda R^{\alpha} \text { for all } x_{0} \in \mathbb{R}^{3} \text { and } R \geq 1\right\} .
$$

We also define

and

$$
A_{\alpha, p}(H)=\max \left[A_{\alpha}(H), A_{\alpha}(H)^{1-\frac{p}{4}}\right]
$$

$$
\mathcal{A}_{\alpha, p}(H)=\max \left[A_{\alpha}(H), A_{\alpha}(H)^{2-\frac{p}{2}}\right] .
$$

Note that if $H_{\tau}$ is a translate of $H$, defined by $H_{\tau}(x)=H(x+\tau)$, then $A_{\alpha}(H)=$ $A_{\alpha}\left(H_{\tau}\right)$ (and, of course, the same is true for $A_{\alpha, p}(H)$ and $\mathcal{A}_{\alpha, p}(H)$ ).

We say $H$ is a weight of dimension $\alpha$ if $\|H\|_{L^{\infty}} \leq 1$ and $A_{\alpha}(H)<\infty$. In this case, any translate $H_{\tau}$ of $H$ is also a weight of dimension $\alpha$.

We alert the reader that since $A_{\beta}(H) \leq A_{\alpha}(H)$ if $\beta \geq \alpha$, a weight $H$ of dimension $\alpha$ is also a weight of dimension $\beta$. So the phrase " $H$ is a weight of dimension $\alpha$ " is just a way of expressing in words the inequality $A_{\alpha}(H)<\infty$, and is not meant to assign the specific dimension $\alpha$ to the function $H$.

The aim of this paper is to prove the following theorem.

Theorem 1.1. Let $S \subset \mathbb{R}^{3}$ be a compact $C^{\infty}$ surface with strictly positive second fundamental form, and $H$ be a weight of dimension $\alpha$.

(i) Suppose $3 / 2 \leq \alpha<5 / 2$. Then to every $\epsilon>0$ there is a constant $C_{\epsilon}(\alpha, S)$ such that

$$
\int_{B(0, R)}|E f(x)|^{p} H(x) d x \leq C_{\epsilon}(\alpha, S) R^{\epsilon} A_{\alpha, p}(H)\|f\|_{L^{2}(S)}^{3}\|f\|_{L^{\infty}(S)}^{p-3}
$$

for all $f \in L^{\infty}(S)$ and $R \geq 1$, where

$$
p=2 \frac{4 \alpha+3}{2 \alpha+3}
$$

(ii) Suppose $3 / 2 \leq \alpha<2$. Then to every $\epsilon>0$ there is a constant $C_{\epsilon}(\alpha, S)$ such that

$$
\int_{B(0, R)}|E f(x)|^{3} H(x) d x \leq C_{\epsilon}(\alpha, S) R^{\epsilon} A_{\alpha, 3}(H) R^{\frac{1}{4}\left(\alpha-\frac{3}{2}\right)}\|f\|_{L^{2}(S)}^{3}
$$


for all $f \in L^{2}(S)$ and $R \geq 1$.

(iii) Suppose $5 / 2 \leq \alpha \leq 3$. Let $2 \leq \gamma<(11 / 2)-\alpha$. Then to every $\epsilon>0$ there is a constant $C_{\epsilon}(\alpha, \gamma, S)$ such that

$$
\int_{B(0, R)}|E f(x)|^{p} H(x) d x \leq C_{\epsilon}(\alpha, \gamma, S) R^{\epsilon} \mathcal{A}_{\alpha, p}(H)\|f\|_{L^{2}(S)}^{\gamma}\|f\|_{L^{\infty}(S)}^{p-\gamma}
$$

for all $f \in L^{\infty}(S)$ and $R \geq 1$, where $p=13 / 4$.

For example, if $H=\chi_{\Omega_{1}}$, then

$$
\int_{B_{R}}|E f(x)|^{3} H(x) d x=\int_{B(0, R)}\left|E\left(e^{-2 \pi i \tau \cdot \xi} f\right)(x)\right|^{3} H(x+\tau) d x,
$$

where $\tau$ is the center of $B_{R}$. Applying part (i) (or part (ii)) of Theorem 1.1 with $\alpha=3 / 2$, we prove (3). Similarly, taking $H=\chi_{\Omega_{2}}$ and applying part (i) of Theorem 1.1 with $\alpha=2$, we prove (4).

It would be very interesting to know if the estimates in Theorem 1.1 have counterparts in dimension $n=2$ or $n \geq 4$. For example, if $H$ is a weight on $\mathbb{R}^{n}$ of dimension $\alpha=n / 2$, and $p=2 n /(n-1)$, then do we have

$$
\int_{B(0, R)}|E f(x)|^{p} H(x) d x \leq C_{\epsilon} R^{\epsilon} A_{\alpha, p}(H)\|f\|_{L^{2}(S)}^{p}
$$

for all $f \in L^{2}(S), R \geq 1$, and $\epsilon>0$ ?

The main obstacle in generalizing Theorem 1.1 to higher dimensions is the current unavailability of the needed Kakeya information (in the form of Lemma 8-D) in dimensions four and more. We refer the reader to Conjecture 11.6 in 9 for more information about this important topic. As for an explanation of the reason why the methods of this paper do not seem to apply in dimension $n=2$, we refer the reader to Remark 11.1.

1.2. Methodology. We start with a couple of definitions.

If $\mathcal{L}$ is a set of lines in $\mathbb{R}^{3}$, and $r \geq 2$ is an integer, then the set of $r$-rich points of $\mathcal{L}$ is defined as

$$
P_{r}(\mathcal{L})=\left\{x \in \mathbb{R}^{3}: x \text { belongs to at least } r \text { lines from } \mathcal{L}\right\} .
$$

We denote the zero set of a polynomial $Q$ by $Z(Q)$, and we say $Q$ is non-singular if $\nabla Q(x) \neq 0$ for all $x \in Z(Q)$.

To prove Theorem 1.1 we employ Guth's polynomial partitioning method from [8]. The proof is carried along nine main steps.

Step one translates the geometric properties of the surface $S$ into a decomposition, called the wave packet decomposition, which is applicable to the functions $f \in L^{2}(S)$ and allows one who works locally in the ball $B_{R}$ of center 0 and radius $R$ to think of $E f$ as being essentially supported on long thin tubes of radius $R^{(1 / 2)+\delta}$ for some parameter $\delta$ (which is positive and rather small).

Step two associates to every polynomial $P$ on $\mathbb{R}^{3}$ of degree at most $D$, which is a product of non-singular polynomials, a partitioning of $\mathbb{R}^{3}$ as follows. We know that $\mathbb{R}^{3} \backslash Z(P)$ is a disjoint union of at most $C D^{3}$ open sets $O_{i}$, which are often called cells. We also know that a line can intersect at most $D+1$ of the cells $O_{i}$. We let $W$ be the $R^{(1 / 2)+\delta}$-neighborhood of $Z(P)$. Then $\mathbb{R}^{n} \backslash W$ is a disjoint union of the open sets $O_{i}^{\prime}=O_{i} \backslash W$, and a tube of radius $R^{(1 / 2)+\delta}$ can intersect at most $D+1$ of the modified cells $O_{i}^{\prime}$. 
Step three organizes those tubes from step one that meet $W$ into two groups: the transverse tubes are those tubes that intersect $W$ transversally, and the tangential tubes are those that lie in $W$ over a long stretch.

Step four identifies the part of $E f$ corresponding to those tubes that point in different directions. Guth calls this the broad part of $E f$. The relation between the broad part of $E f$ and the long thin tubes that support $E f$ resembles the relation between the set $P_{r}(\mathcal{L})$, as defined at the start of this subsection, and the lines of $\mathcal{L}$. This is the main reason behind the polynomial method becoming as important in restriction theory as it has become in incidence geometry. We refer the reader to Subsections 0.4 and 0.5 of 8 for a thorough discussion about the common features between bounding the number of $r$-rich points of $\mathcal{L}$ and estimating the broad part of $E f$.

Step five formulates a theorem, Theorem 9.1 that estimates the $L^{p}(H d x)$ norm of the broad part of $E f$ on $B_{R}$ conditional on having a favorable bound on the contribution coming from the tangential tubes. The conditional bound on the tangential tubes is uniform over all polynomials $P$ satisfying $\operatorname{Deg}(P) \leq D$ (for some specified $D$ ), and $P$ is a product of non-singular polynomials. This conditional formulation of Theorem 9.1 resembles the formulation of incidence geometry theorems in 7 and 10 that estimate $\left|P_{r}(\mathcal{L})\right|$ conditional on having a favorable bound on the number of lines of $\mathcal{L}$ that lie in $Z(P)$ (with the conditional bound being uniform over all $P$ satisfying appropriate properties).

Step six proves Theorem 9.1. We first find (using algebraic topology) an appropriate polynomial $P$ such that the modified cells $O_{i}^{\prime}$, which were associated to $P$ in step two, contribute roughly equally to the $L^{p}(H d x)$ norm of the broad part of $E f$ over $B_{R}$. This will then allow us to bound the contribution that comes from the cells by induction on the "size" of the function $f$, and the contribution from the transverse tubes by induction on the radius $R$.

Step seven bounds the contribution from the tangential tubes. The tangential tubes gather rather close to an algebraic surface and dealing with them becomes roughly a two dimensional problem. In the incidence geometry setting, to bound the number of lines of $\mathcal{L}$ that lie in $Z(P)$, one uses the available geometric properties of the lines of $\mathcal{L}$. In the Fourier restriction setting, to bound the contribution from the tubes tangent to $Z(P)$, one uses the available Kakeya information. In this paper, the Kakeya information we use are contained in Lemma 8-D, which was proved in 8] by adapting Wolff's hairbrush argument to the polynomial partitioning setting.

Step eight inserts the bounds from step seven into Theorem 9.1 obtaining estimates on the $L^{p}(H d x)$ norm of the broad part of $E f$ over $B_{R}$ (see Theorems 11.1 and 11.2).

Step nine uses parabolic scaling and induction on the radius $R$ to upgrade the estimates we now have on the $L^{p}(H d x)$ norm of the broad part of $E f$ on $B_{R}$ into estimates on the $L^{p}(H d x)$ norm of $E f$ itself, proving Theorem 1.1.

Steps one to four are more or less identical to the treatment in [8]. Steps five to nine differ from the treatment in [8] in the following aspects.

The estimate on the broad part of $E f$ (steps five, six, and eight) is established in $\left[8\right.$, in the non-weighted setting (i.e., $H(x)=1$ for all $x \in \mathbb{R}^{3}$ ) and for functions $f$ that obey the condition

$$
\int_{B\left(\xi_{0}, R^{-1 / 2}\right) \cap S}|f(\xi)|^{2} d \sigma(\xi) \leq \frac{1}{R}
$$


for all $\xi_{0} \in S$. In this paper, we let $b \geq 1$ be a parameter and establish the estimate on the broad part in the weighted setting for functions $f$ that obey the condition

$$
\int_{B\left(\xi_{0}, R^{-1 / 2}\right) \cap S}|f(\xi)|^{2} d \sigma(\xi) \leq \frac{1}{R^{(b+1) / 2}}
$$

for all $\xi_{0} \in S$. To prove parts (i) and (iii) of Theorem 1.1, we let $b=1$ later in the argument. To prove part (ii) of Theorem 1.1, we pick for $b$ a large value that depends on $\epsilon$. We alert the reader to the fact that even though the estimate in the case $\alpha=3 / 2$ is stated in both parts (i) and (ii) of Theorem 1.1, its proof belongs to part (ii), and hence requires the large value of $b$.

In bounding the contribution from the tangential tubes (step seven), we adjust the corresponding argument from [8] to take into consideration the dimensionality of the weight $H$, which is reflected in the inequality $\int_{B\left(x_{0}, R\right)} H(x) d x \leq A_{\alpha}(H) R^{\alpha}$. This is where we lower the value of the exponent $p$ from $13 / 4$ to $2(4 \alpha+3) /(2 \alpha+3)$.

The induction argument that 8$]$ uses to upgrade the estimate on the broad part of $E f$ to an estimate on $E f$ itself (step nine), assumes in the induction hypothesis that the desired estimate holds for all the surfaces in $\mathbb{R}^{3}$ that have the same geometric properties as the given surface $S$, and then uses parabolic scaling. The induction hypothesis in this paper assumes that the desired estimate holds not only for all the surfaces in $\mathbb{R}^{3}$ that have the same geometric properties as $S$, but also for all the weights on $\mathbb{R}^{3}$ of dimension $\alpha$. Then, to carry the induction out, the parabolic scaling argument gets adjusted accordingly. This turns out to be a little more involved than the corresponding argument in 8 .

1.3. Notation. Throughout this paper, a closed ball in $\mathbb{R}^{3}$ of center $x$ and radius $r$ will be denoted by $B(x, r)$. A closed ball in $\mathbb{R}^{2}$ of center $\omega$ and radius $r$ will be denoted by $B^{2}(x, r)$. For example, $B^{2}(0,1)$ is the closed unit ball in the plane.

If $A$ and $B$ are two positive quantities, then $A \lesssim B$ means that $A \leq C B$ for a suitable constant $C$. If $A \lesssim B$ and $B \lesssim A$, then we write $A \sim B$.

If $\phi$ is a function on $\mathbb{R}^{n}$ and $r$ is a positive number, then $\phi_{r}$ will denote the function defined by $\phi_{r}(x)=r^{-n} \phi\left(r^{-1} x\right)$. Also, if $\Theta$ is a ball in $\mathbb{R}^{n}$, then $r \Theta$ will denote the ball of the same center as $\Theta$ and $r$ times the radius.

1.4. Acknowledgment. The author wishes to thank the anonymous referee for many insightful suggestions concerning the structure of this paper.

\section{Global Restriction estimates}

Some of our local restriction estimates can be turned into global estimates. The tool for doing this is Tao's $\epsilon$-removal lemma from [15]. When one goes over the proof of Tao's $\epsilon$-removal lemma (especially as presented in [4]), one sees that the proof can be carried over to the weighted setting of this paper when $H$ is the characteristic function of a set of the form

$\Omega_{a, b}= \begin{cases}\cup_{(m, n) \in \mathbb{Z}^{2}}\left(\mathbb{R} \times[-1,1]^{2}+\left(0,(\operatorname{sgn} m)|m|^{1 / a},(\operatorname{sgn} n)|n|^{1 / b}\right)\right) & \text { if } 0<a, b \leq 1, \\ \cup_{m \in \mathbb{Z}}\left(\mathbb{R} \times[-1,1]^{2}+\left(0,(\operatorname{sgn} m)|m|^{1 / a}, 0\right)\right) & \text { if } 0<a \leq 1 \text { and } b=0, \\ \cup_{n \in \mathbb{Z}}\left(\mathbb{R} \times[-1,1]^{2}+\left(0,0,(\operatorname{sgn} n)|n|^{1 / b}\right)\right) & \text { if } a=0 \text { and } 0<b \leq 1,\end{cases}$ 
where $\operatorname{sgn} m=m /|m|$ if $m \neq 0$, and $\operatorname{sgn} 0=1$. We note that

$$
\int_{B\left(x_{0}, R\right)} \chi_{\Omega_{a, b}}(x) d x \lesssim R^{1+a+b}
$$

for all $x_{0} \in \mathbb{R}^{3}$ and $R \geq 1$, so that $A_{\alpha}\left(\chi_{\Omega_{a, b}}\right) \lesssim 1$ if $a+b=\alpha-1$. Also, $\Omega_{1,1}=\mathbb{R}^{3}$.

The restriction conjecture (in its global form) in $\mathbb{R}^{3}$ asserts that the estimate

$$
\|E f\|_{L^{p}\left(\mathbb{R}^{3}\right)} \leq C(p, q, S)\|f\|_{L^{q}(S)}
$$

holds whenever $p>3,(2 / p)+(1 / q) \leq 1$, and $f \in L^{q}(S)$. This estimate immediately implies that the Fourier transform of any function $f \in L^{(3 / 2)-\epsilon}\left(\mathbb{R}^{3}\right)$ can be restricted to $S$. So long as this conjecture remains unsolved, it is natural to ask if there is a set $\Omega$ in $\mathbb{R}^{3}$ of infinite Lebesgue measure such that the Fourier transform of any function $f \in L^{(3 / 2)-\epsilon}(\Omega)$ can be restricted to $S$. Taking $\Omega=\Omega_{1 / 4,1 / 4}$, part (i) of the following corollary tells us that this is indeed the case.

Corollary 2.1. Let $S \subset \mathbb{R}^{3}$ be a compact $C^{\infty}$ surface with strictly positive second fundamental form.

(i) Suppose $3 / 2 \leq \alpha<5 / 2, p_{0}=4(4 \alpha+3) /(10 \alpha+3)$, and $p>2(4 \alpha+3) /(2 \alpha+3)$. Then

$$
\|E f\|_{L^{p}\left(\Omega_{a, b}\right)} \leq C(\alpha, p, S)\|f\|_{L^{p_{0}^{\prime}(S)}}
$$

for all $f \in L^{p_{0}^{\prime}}(S)$ provided $a+b=\alpha-1$, where $p_{0}^{\prime}$ is the exponent conjugate to $p_{0}$.

(ii) Suppose $5 / 2 \leq \alpha \leq 3,1 \leq p_{0}<13 /(2+2 \alpha)$, and $p>13 / 4$. Then

$$
\|E f\|_{L^{p}\left(\Omega_{a, b}\right)} \leq C\left(\alpha, p_{0}, p, S\right)\|f\|_{L^{p_{0}^{\prime}(S)}}
$$

for all $f \in L^{p_{0}^{\prime}}(S)$ provided $a+b=\alpha-1$.

(iii) We have

$$
\|E f\|_{L^{p}\left(\mathbb{R}^{3}\right)} \leq C(p, q, S)\|f\|_{L^{q}(S)}
$$

whenever $p>13 / 4,(2 / p)+(1 / q)<1$, and $f \in L^{q}(S)$.

Part (iii) of Corollary 2.1 is a modest improvement on Guth's global restriction estimate from [8]; in Guth's theorem, the norm on the right-hand side of the inequality is an $L^{\infty}$ norm. At any rate, part (iii) of Corollary 2.1 proves a restriction theorem in $\mathbb{R}^{3}$ for $p>3.25$ in the full conjectured range of exponents up to the sharp line $(2 / p)+(1 / q)=1$. Also, part (i) of Corollary 2.1 proves the global version of (4):

$$
\|E f\|_{L^{p}\left(\Omega_{2}\right)}=\|E f\|_{L^{p}\left(\Omega_{1,0}\right)} \lesssim\|f\|_{L^{44 / 21}(S)} \quad(p>22 / 7) .
$$

\section{Applications in geometric measure theory}

Fourier restriction theory has well-known and important implications to an area of study that lies on the boundary between harmonic analysis and geometric measure theory, and revolves around Falconer's distance set conjecture.

Let $K$ be a compact subset of $\mathbb{R}^{n}, n \geq 2$. The distance set of $K$ is defined as

$$
\Delta(K)=\{|x-y|: x, y \in K\} .
$$

Falconer's conjecture asserts that if $K$ has Hausdorff dimension greater than $n / 2$, then $\Delta(K)$ has positive (one-dimensional) Lebesgue measure.

Falconer initiated the study of the connection between Hausdorff dimension and distance sets in [6] and used Fourier analysis (via potential theory) to show that if the Hausdorff dimension of $K$ is greater than $(n+1) / 2$, then $\Delta(K)$ has positive 
Lebesgue measure. The Fourier analytic approach to studying this problem was developed further by Mattila in [12, where getting information about the Lebesgue measure of $\Delta(K)$ was linked to obtaining favorable decay estimates as $R \rightarrow \infty$ on integrals of the form

$$
\int|\widehat{\mu}(R \xi)|^{2} d \sigma_{n-1}(\xi)
$$

for $\mu \in M\left(\mathbb{R}^{n}\right)$, where $\sigma_{n-1}$ is the surface measure on the unit sphere $\mathbb{S}^{n-1} \subset \mathbb{R}^{n}$ and $M\left(\mathbb{R}^{n}\right)$ is the space of all complex Borel measures on $\mathbb{R}^{n}$. Of course, favorable decay estimates on such integrals cannot be obtained for general $\mu \in M\left(\mathbb{R}^{n}\right)$. In fact, on one end of the spectrum we have Dirac measures, for which the above integral is equal to $\sigma_{n-1}\left(\mathbb{S}^{n-1}\right)$ for all $R$. On the other end of the spectrum we have the absolutely continuous measures with Schwartz densities, whose Fourier transforms decay like $C_{N} R^{-N}$ for any positive integer $N$.

Suppose $K \subset \mathbb{R}^{n}$ is compact and has Hausdorff dimension $\alpha$. It is well known (e.g., see [21]) that if $\alpha<\beta<n$, then $K$ supports a probability measure $\mu$ that satisfies

$$
\mu(B(x, r)) \leq C r^{\beta}
$$

for a suitable constant $C$ and all $x \in \mathbb{R}^{n}$ and $r>0$. It is also well known that this condition implies that the measure's $\alpha$-dimensional energy $I_{\alpha}(\mu)$, defined as

$$
I_{\alpha}(\mu)=\iint \frac{1}{|x-y|^{\alpha}} d \mu(x) d \mu(y),
$$

is finite. The $\alpha$-dimensional energy has the following Fourier representation:

$$
I_{\alpha}(\mu)=c_{\alpha} \int_{\mathbb{R}^{n}}|\widehat{\mu}(\eta)|^{2}|\eta|^{\alpha-n} d \eta,
$$

where $c_{\alpha}$ is a constant that depends only on $\alpha$ and $n$. Moving to polar coordinates, we see that

$$
\int_{\mathbb{R}^{n}}|\widehat{\mu}(\eta)|^{2}|\eta|^{\alpha-n} d \eta=\int_{0}^{\infty}\left(\int|\widehat{\mu}(R \xi)|^{2} d \sigma_{n-1}(\xi)\right) R^{\alpha-1} d R,
$$

and so we expect the finiteness of the $\alpha$-dimensional energy of $\mu$ to lead to some control over $\int|\widehat{\mu}(R \xi)|^{2} d \sigma_{n-1}(\xi)$ as $R \rightarrow \infty$. Naturally, the tighter the control we have over these integrals, the better the result we obtain on Falconer's conjecture by using the methods of 12 .

In view of Mattila's work, Bourgain brought restriction theory into the picture in [3], where he showed that Falconer's $(n+1) / 2$ result follows from the Tomas-Stein restriction estimate, and used the better restriction estimates that were available in dimensions two and three to improve on Falconer's result. Bourgain showed that if $K \subset \mathbb{R}^{2}$ has Hausdorff dimension greater than $13 / 9$, then $\Delta(K)$ has positive Lebesgue measure, and that if $K \subset \mathbb{R}^{3}$ has Hausdorff dimension greater than $1091 / 546=1.998 \ldots$, then $\Delta(K)$ has positive Lebesgue measure.

The next improvement came in [19, where Wolff showed that if $K \subset \mathbb{R}^{2}$ has dimension greater than $4 / 3$, then $\Delta(K)$ has positive Lebesgue measure. Wolff got

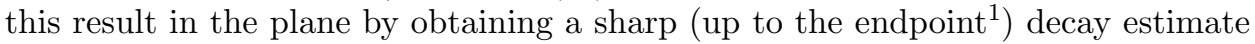
on the circular means $\int|\widehat{\mu}(R \xi)|^{2} d \sigma_{1}(\xi)$. Wolff proved that if $1 \leq \alpha<2$, and $\mu$ is a

\footnotetext{
${ }^{1}$ It is not known whether the estimate (7) holds without the $R^{\epsilon}$ factor.
} 
positive measure in $M\left(\mathbb{R}^{2}\right)$ with support in the unit disc and finite $\alpha$-dimensional energy, then

$$
\int|\widehat{\mu}(R \xi)|^{2} d \sigma_{1}(\xi) \leq C_{\epsilon} \frac{R^{\epsilon}}{R^{\frac{\alpha}{2}}} I_{\alpha}(\mu)
$$

for all $R \geq 1$. Wolff also considered the $L^{q}$ circular means $\int|\widehat{\mu}(R \xi)|^{q} d \sigma_{1}(\xi)$ and observed that for $q>2$ one cannot do better than interpolating between the above $L^{2}$ estimate and the trivial $L^{\infty}$ estimate. The case $1 \leq q<2$, however, presented a different challenge. The only estimate in this case came from applying Hölder's inequality and using (7), and it remains an open problem to determine whether or not better estimates are available. As was also explained in [19, estimates for the $L^{1}$ means are particularly interesting as they are related to the open problem of evaluating the infimum of the Hausdorff dimension of $\beta$-sets. We refer the reader to [19] (and [1]) for more details.

Using Tao's bilinear restriction estimate [16, Erdoğan studied the same problem in higher dimensions and proved in [5] that if $n / 2 \leq \alpha \leq(n+2) / 2$, and $\mu \in M\left(\mathbb{R}^{n}\right)$ is positive with supp $\mu$ contained in the unit ball and $I_{\alpha}(\mu)<\infty$, then

$$
\int|\widehat{\mu}(R \xi)|^{2} d \sigma_{n-1}(\xi) \leq C_{\epsilon} \frac{R^{\epsilon}}{R^{\frac{\alpha}{2}+\frac{n}{4}-\frac{1}{2}}} I_{\alpha}(\mu)
$$

for all $R \geq 1$. This estimate gives the currently best known result on Falconer's conjecture: if $K \subset \mathbb{R}^{n}$ has Hausdorff dimension greater that $(n / 2)+(1 / 3)$, then $\Delta(K)$ has positive Lebesgue measure. Once again, for $1 \leq q<2$, the best known estimate on the $L^{q}$ spherical means is the one we get from Hölder's inequality and (8). For example, in dimension $n=3$, we have

$$
\left(\int|\widehat{\mu}(R \xi)|^{q} d \sigma_{2}(\xi)\right)^{1 / q} \leq C_{\epsilon} \frac{R^{\epsilon}}{R^{\frac{\alpha}{4}+\frac{1}{8}}} \sqrt{I_{\alpha}(\mu)}
$$

for all $R \geq 1$, provided $3 / 2 \leq \alpha \leq 5 / 2$.

As a corollary to Theorem 1.1 we obtain the following result concerning the $L^{q}$ norm of $\widehat{\mu}(R \cdot)$ on $S$ for $R \geq 1$.

Corollary 3.1. Let $S \subset \mathbb{R}^{3}$ be a compact $C^{\infty}$ surface with strictly positive second fundamental form, and $\mu$ be a positive measure in $M\left(\mathbb{R}^{3}\right)$ with support in the closed unit ball and finite $\alpha$-dimensional energy.

(i) Suppose $3 / 2 \leq \alpha<5 / 2, p=2(4 \alpha+3) /(2 \alpha+3)$, and $p_{0}=2 p /(2 p-3)=$ $4(4 \alpha+3) /(10 \alpha+3)$. Then to every $\epsilon>0$ there is a constant $C_{\epsilon}(\alpha, S)$ such that

$$
\|\widehat{\mu}(R \cdot)\|_{L^{p_{0}}(S)} \leq C_{\epsilon}(\alpha, S) R^{\epsilon} R^{-\alpha / p} \sqrt{I_{\alpha}(\mu)}
$$

for all $R \geq 1$.

(ii) Suppose $3 / 2 \leq \alpha<2$. Then to every $\epsilon>0$ there is a constant $C_{\epsilon}(\alpha, S)$ such that

$$
\|\widehat{\mu}(R \cdot)\|_{L^{2}(S)} \leq C_{\epsilon}(\alpha, S) R^{\epsilon} R^{-(\alpha / 4)-(1 / 8)} \sqrt{I_{\alpha}(\mu)}
$$

for all $R \geq 1$.

\footnotetext{
${ }^{2}$ There are available estimates for the $L^{2}$ spherical means of the Fourier transforms of such measures in the complementary range $\alpha \in(0, n / 2) \cup((n+2) / 2, n)$, which are known to be sharp (up to the endpoint) only for $0<\alpha \leq(n-1) / 2$. In dimension $n=2$, however, these estimates are known to be sharp (again up to the endpoint) for all $0<\alpha<2$; see [12, 19], and 14.
} 
(iii) Suppose $5 / 2 \leq \alpha<13 / 5$ and $1 \leq p_{0}<13 /(2+2 \alpha)$. Then to every $\epsilon>0$ there is a constant $C_{\epsilon}\left(\alpha, p_{0}, S\right)$ such that

$$
\|\widehat{\mu}(R \cdot)\|_{L^{p_{0}}(S)} \leq C_{\epsilon}\left(\alpha, p_{0}, S\right) R^{\epsilon} R^{-4 \alpha / 13} \sqrt{I_{\alpha}(\mu)}
$$

for all $R \geq 1$.

The estimates in parts (i) and (iii) of Corollary 3.1 are new and form one of the main results of this paper. This is the first time an estimate on the $L^{1}$ spherical means of $\widehat{\mu}$ goes beyond what is known on the $L^{2}$ spherical means.

Part (iii) is, in fact, true for $5 / 2 \leq \alpha<3$. We state it as above, because in the regime $13 / 5 \leq \alpha<3$ it becomes inferior to the known estimate $\|\widehat{\mu}(R \cdot)\|_{L^{2}(S)} \lesssim$ $R^{(1-\alpha) / 2} \sqrt{I_{\alpha}(\mu)}$ (see [14] or [21]).

As we mentioned before the statement of the corollary, the result of part (ii) of Corollary 3.1 has previously been obtained in 5 by using Tao's bilinear restriction estimate from [16]. It gives the best known result on Falconer's conjecture in $\mathbb{R}^{3}$, and now has a proof that is based on polynomial partitioning.

After this paper was written, the author learned about the paper [11, which obtains new results about the decay rate of $\|\widehat{\mu}(R \cdot)\|_{L^{2}\left(\mathbb{S}^{n-1}\right)}$. In dimension $n=3$, the results of [11] are better than Corollary 3.1 when $\alpha>(8+\sqrt{85}) / 7 \approx 2.46$ and $S$ is the unit sphere $\mathbb{S}^{2}$.

\section{An estimate for eXPonential sums}

We can impose a slightly different condition on the measure than having a finite $\alpha$-dimensional energy. Namely,

$$
\sup _{x \in \mathbb{R}^{3}} \sup _{r>0} \frac{\mu(B(x, r))}{r^{\alpha}}<\infty .
$$

This condition on the measure is morally stronger than having finite $\alpha$-dimensional energy. In fact, a result that the author learned from Wolff's paper 19] (which is also stated below as Lemma 6-B) says that, for $R \geq 1$, a positive compactly supported measure $\mu \in M\left(\mathbb{R}^{3}\right)$ with finite $\alpha$-dimensional energy can be decomposed as a sum of $O(1+\log R)$ measures $\mu_{j}$ satisfying

$$
\sup _{x \in \mathbb{R}^{3}} \sup _{r \geq R^{-1}} \frac{\mu(B(x, r))}{r^{\alpha}}<\infty .
$$

Under (9), $\mu$ itself satisfies (10) with a uniform bound for all $R \geq 1$.

The next corollary says that under (9)), Theorem 1.1 gives $L^{p}(\mu)$ bounds on exponential sums of the form $\sum_{l=1}^{N} a_{l} e^{2 \pi i R w_{l} \cdot x}$, where $w_{1}, \ldots, w_{N} \in S$ are $R^{-1}$ separated.

Corollary 4.1. Let $S \subset \mathbb{R}^{3}$ be a compact $C^{\infty}$ surface with strictly positive second fundamental form, and $\mu$ be a positive measure in $M\left(\mathbb{R}^{3}\right)$ with support in the closed unit ball. Also, let

$$
\mathcal{C}_{\alpha}(\mu)=\sup _{x \in \mathbb{R}^{3}} \sup _{r>0} \frac{\mu(B(x, r))}{r^{\alpha}} .
$$

(i) Suppose $3 / 2 \leq \alpha<5 / 2$ and $p=2(4 \alpha+3) /(2 \alpha+3)$. Then to every $\epsilon>0$ there is a constant $C_{\epsilon}(\alpha, S)$ such that

$$
\int\left|\sum_{l=1}^{N} a_{l} e^{2 \pi i R w_{l} \cdot x}\right|^{p} d \mu(x) \leq C_{\epsilon}(\alpha, S) \frac{R^{\epsilon} R^{2 p}}{R^{\alpha+3}} \mathcal{C}_{\alpha}(\mu)\left(\sum_{l=1}^{N}\left|a_{l}\right|^{2}\right)^{3 / 2}\left(\max _{l}\left|a_{l}\right|\right)^{p-3}
$$


whenever $R \geq 1, w_{1}, \ldots, w_{N} \in S$ are $R^{-1}$-separated, and $a_{1}, \ldots, a_{N} \in \mathbb{C}$.

(ii) Suppose $5 / 2 \leq \alpha \leq 3,2 \leq \gamma<(11 / 2)-\alpha$, and $p=13 / 4$. Then to every $\epsilon>0$ there is a constant $C_{\epsilon}(\alpha, \gamma, S)$ such that

$$
\begin{aligned}
& \int\left|\sum_{l=1}^{N} a_{l} e^{2 \pi i R w_{l} \cdot x}\right|^{p} d \mu(x) \\
& \leq C_{\epsilon}(\alpha, \gamma, S) \frac{R^{\epsilon} R^{2 p}}{R^{\alpha+\gamma}} \mathcal{C}_{\alpha}(\mu)\left(\sum_{l=1}^{N}\left|a_{l}\right|^{2}\right)^{\gamma / 2}\left(\max _{l}\left|a_{l}\right|\right)^{p-\gamma}
\end{aligned}
$$

whenever $R \geq 1, w_{1}, \ldots, w_{N} \in S$ are $R^{-1}$-separated, and $a_{1}, \ldots, a_{N} \in \mathbb{C}$.

Parts (i) and (ii) of Corollary 4.1 are sharp (up to the $R^{\epsilon}$ factor) in the following sense. Take $\mu$ to be the restriction of an Ahlfors $\alpha$-regular measure $\nu$ to the unit ball, $a_{l}=1$ for all $l$, and $N \sim R^{2}$. Being Ahlfors $\alpha$-regular means that there are positive constants $C_{1}$ and $C_{2}$ such that $C_{1} r^{\alpha} \leq \nu(B(x, r)) \leq C_{2} r^{\alpha}$ for all $x \in \mathbb{R}^{3}$ and $r>0$. Then $\mathcal{C}_{\alpha}(\mu) \leq C_{2}$ and Corollary 4.1 implies that

$$
\frac{R^{2 p}}{R^{\alpha}} \lesssim \int_{B\left(0, c R^{-1}\right)}\left|\sum_{l=1}^{N} e^{2 \pi i R w_{l} \cdot x}\right|^{p} d \mu(x) \leq \int\left|\sum_{l=1}^{N} e^{2 \pi i R w_{l} \cdot x}\right|^{p} d \mu(x) \lesssim R^{\epsilon} \frac{R^{2 p}}{R^{\alpha}},
$$

where $c$ is an appropriately small constant. For example, when $\alpha=3 / 2$ this becomes

$$
R^{9 / 2} \lesssim \int\left|\sum_{l=1}^{N} e^{2 \pi i R w_{l} \cdot x}\right|^{3} d \mu(x) \lesssim R^{\epsilon} R^{9 / 2}
$$

\section{Preliminaries FOR the Proofs of the COROllaries}

The global restriction estimates of Corollary 2.1 will be proved by combining the local estimates of Theorem 1.1] with Tao's $\epsilon$-removal lemma from [15. To apply the $\epsilon$-removal lemma, however, it will be convenient to free the estimates in parts (i) and (iii) of Theorem 1.1 from the $L^{\infty}$-norm of $f$. It will also be convenient to write the estimates in their dual form. This is the goal of the following theorem; which will also be important to the proof of Corollary 3.1 .

Theorem 5.1. Let $S \subset \mathbb{R}^{3}$ be a compact $C^{\infty}$ surface with strictly positive second fundamental form, and $H$ be a weight of dimension $\alpha$ with $A_{\alpha}(H) \leq 1$.

(i) Suppose $3 / 2 \leq \alpha<5 / 2, p=2(4 \alpha+3) /(2 \alpha+3)$, and $p_{0}=4(4 \alpha+3) /(10 \alpha+3)$. Then to every $\epsilon>0$ there is a constant $C_{\epsilon}(\alpha, S)$ such that

$$
\|\mathcal{R} f\|_{L^{p_{0}(S)}} \leq C_{\epsilon}(\alpha, S) R^{\epsilon}\|f\|_{L^{p^{\prime}}\left(\chi_{B(0, R)} H d x\right)}
$$

whenever $R \geq 1$ and $f \in L^{p^{\prime}}\left(\chi_{B(0, R)} H d x\right)$, where $\mathcal{R} f=\left.\widehat{f H}\right|_{S}$ and $p^{\prime}$ is the exponent conjugate to $p$.

(ii) Suppose $5 / 2 \leq \alpha \leq 3, p=13 / 4$, and $1 \leq p_{0}<13 /(2+2 \alpha)$. Then to every $\epsilon>0$ there is a constant $C_{\epsilon}\left(\alpha, p_{0}, S\right)$ such that

$$
\|\mathcal{R} f\|_{L^{p_{0}(S)}} \leq C_{\epsilon}\left(\alpha, p_{0}, S\right) R^{\epsilon}\|f\|_{L^{p^{\prime}}\left(\chi_{B(0, R)} H d x\right)}
$$

whenever $R \geq 1$ and $f \in L^{p^{\prime}}\left(\chi_{B(0, R)} H d x\right)$, where $\mathcal{R} f=\left.\widehat{f H}\right|_{S}$ and $p^{\prime}=13 / 9$. 
Proof. Since $A_{\alpha}(H) \leq 1$, parts (i) and (iii) of Theorem 1.1 can be combined as

$$
\int_{B(0, R)}|E g(x)|^{\bar{p}} H(x) d x \lesssim R^{\epsilon}\|g\|_{L^{2}(S)}^{\bar{\gamma}}\|g\|_{L^{\infty}(S)}^{\bar{p}-\bar{\gamma}}
$$

for all $g \in L^{\infty}(S)$, where

$$
\bar{p}=\left\{\begin{array}{ll}
2(4 \alpha+3) /(2 \alpha+3) & \text { if } \frac{3}{2} \leq \alpha<\frac{5}{2}, \\
13 / 4 & \text { if } \frac{5}{2} \leq \alpha \leq 3,
\end{array} \quad \text { and } \quad \bar{\gamma}= \begin{cases}3 & \text { if } \frac{3}{2} \leq \alpha<\frac{5}{2}, \\
\gamma & \text { if } \frac{5}{2} \leq \alpha \leq 3\end{cases}\right.
$$

By the duality relation of the Fourier transform and Hölder's inequality, the above estimate tells us that

$$
\begin{aligned}
\left|\int \mathcal{R} f(\xi) g(\xi) d \sigma(\xi)\right| & \leq\|f\|_{L^{\bar{p}^{\prime}}\left(\chi_{B(0, R)} H d x\right)}\|E g\|_{L^{\bar{p}}\left(\chi_{B(0, R)} H d x\right)} \\
& \lesssim R^{\epsilon / \bar{p}}\|f\|_{L^{\bar{p}^{\prime}}\left(\chi_{B(0, R)} H d x\right)}\|g\|_{L^{2}(S)}^{\bar{\gamma} / \bar{p}}\|g\|_{L^{\infty}(S)}^{1-(\bar{p} / \bar{p})}
\end{aligned}
$$

for all $f \in L^{\bar{p}^{\prime}}\left(\chi_{B(0, R)} H d x\right)$ and $g \in L^{\infty}(S)$.

We will now use (11) to prove the theorem. We will do this by estimating the $\sigma$-measure of the sets $\{\xi \in S:|\mathcal{R} f(\xi)|>\lambda\}$ for $0<\lambda \leq\|f\|_{L^{1}\left(\chi_{B(0, R)} H d x\right)}$. For such $\lambda$ and for $l \in \mathbb{N}$, we set

$$
A_{l}=A_{l}(\lambda)=\left\{\xi \in S: 2^{l-1} \lambda<|\mathcal{R} f(\xi)| \leq 2^{l} \lambda\right\} .
$$

Clearly, $\{\xi \in S:|\mathcal{R} f(\xi)|>\lambda\} \subset \cup_{l} A_{l}$. Inserting $\overline{\mathcal{R} f(\xi)} \chi_{A_{l}}(\xi)$ for $g(\xi)$ in (11), we obtain

$$
\left(\int_{A_{l}}|\mathcal{R} f(\xi)|^{2} d \sigma(\xi)\right)^{1-\frac{\bar{\gamma}}{2 \bar{p}}} \lesssim R^{\epsilon / \bar{p}}\|f\|_{L^{\bar{p}^{\prime}}\left(\chi_{B(0, R)} H d x\right)}\left(2^{l} \lambda\right)^{1-(\bar{\gamma} / \bar{p})}
$$

which implies

$$
\sigma\left(A_{l}\right) \lesssim R^{2 \epsilon /(2 \bar{p}-\bar{\gamma})}\|f\|_{L^{\bar{p}^{\prime}}\left(\chi_{B(0, R)} \bar{p} /(\bar{p}-\bar{\gamma})\right.}\left(2^{l} \lambda\right)^{-2 \bar{p} /(2 \bar{p}-\bar{\gamma})} .
$$

Since $3<\bar{p} \leq 13 / 4$ and $2 \leq \bar{\gamma} \leq 3$, it follows that

$$
\sigma(\{\xi \in S:|\mathcal{R} f(\xi)|>\lambda\}) \leq \sum_{l} \sigma\left(A_{l}\right) \lesssim\left(\frac{R^{\epsilon / \bar{p}}\|f\|_{L_{\bar{p}^{\prime}}\left(\chi_{B(0, R)} H d x\right)}}{\lambda}\right)^{p_{0}},
$$

where $p_{0}=2 \bar{p} /(2 \bar{p}-\bar{\gamma})$. Of course, we also have the trivial bound

$$
\sigma(\{\xi \in S:|\mathcal{R} f(\xi)|>\lambda\}) \leq \sigma(S) \lesssim 1 .
$$

We can now bound our integral. We let

$$
\lambda_{0}=R^{\epsilon / \bar{p}}\|f\|_{L^{\bar{p}^{\prime}}\left(\chi_{B(0, R)} H d x\right)} \quad \text { and } \quad \lambda_{1}=\|f\|_{L^{1}\left(\chi_{B(0, R)} H d x\right)},
$$

and we observe that

$$
\int_{0}^{\lambda_{1}} \sigma(\{\xi \in S: \mid \mathcal{R} f(\xi)>\lambda\}) \lambda^{p_{0}-1} d \lambda \lesssim \int_{0}^{\lambda_{0}} \lambda^{p_{0}-1} d \lambda=\frac{\lambda_{0}^{p_{0}}}{p_{0}}
$$

if $\lambda_{1} \leq \lambda_{0}$, and

$$
\begin{aligned}
\int_{0}^{\lambda_{1}} \sigma(\{\xi \in S: \mid \mathcal{R} f(\xi)>\lambda\}) \lambda^{p_{0}-1} d \lambda & \lesssim \int_{0}^{\lambda_{0}} \lambda^{p_{0}-1} d \lambda+\lambda_{0}^{p_{0}} \int_{\lambda_{0}}^{\lambda_{1}} \frac{d \lambda}{\lambda} \\
& \lesssim \lambda_{0}^{p_{0}}\left(1+\log \frac{\lambda_{1}}{\lambda_{0}}\right)
\end{aligned}
$$


if $\lambda_{1}>\lambda_{0}$. But, by Hölder's inequality, $\lambda_{1} \leq|B(0, R)|^{1 / \bar{p}} R^{-\epsilon / \bar{p}} \lambda_{0} \lesssim R \lambda_{0}$, so

$$
\int_{0}^{\lambda_{1}} \sigma(\{\xi \in S: \mid \mathcal{R} f(\xi)>\lambda\}) \lambda^{p_{0}-1} d \lambda \lesssim \lambda_{0}^{p_{0}}(1+\log R) .
$$

Thus

$$
\left(\int|\mathcal{R} f(\xi)|^{p_{0}} d \sigma(\xi)\right)^{1 / p_{0}} \lesssim R^{\epsilon}\|f\|_{L^{\bar{p}^{\prime}}\left(\chi_{B(0, R)} H d x\right)} .
$$

When $3 / 2 \leq \alpha<5 / 2$, we have $\bar{p}=2(4 \alpha+3) /(2 \alpha+3)$ and $\bar{\gamma}=3$, so that $p_{0}=4(4 \alpha+3) /(10 \alpha+3)$. This proves part (i) of the theorem.

When $5 / 2 \leq \alpha \leq 3$, we have $\bar{p}=13 / 4$ and $2 \leq \bar{\gamma}=\gamma<(11 / 2)-\alpha$, so that $13 / 9 \leq p_{0}<13 /(2+2 \alpha)$. This proves part (ii).

We now prove a lemma that is important for our results concerning the decay of the $L^{q}(S)$ means of Fourier transforms of measures, as well as for our estimate on exponential sums. This lemma will allow us to bound $\|E f(R \cdot)\|_{L^{p}(\mu)}$ by $\|E g\|_{L^{p}(H d x)}$ with $|g| \leq|f|$, and for an appropriate weight $H$ that is supported in the ball $B(0,2 R)$.

Lemma 5.1. Suppose $\mu \in M\left(\mathbb{R}^{3}\right)$ is positive and supported in $B(0,1), 0<\alpha \leq 3$, $R \geq 1$, and

$$
\mathcal{C}_{\alpha, R}(\mu)=\sup _{x \in \mathbb{R}^{3}} \sup _{r \geq R^{-1}} \frac{\mu(B(x, r))}{r^{\alpha}} .
$$

Then there is a weight $H$ (which depends on $R$ ) of dimension $\alpha$ such that

(i) $A_{\alpha}(H) \leq|B(0,1)|$

(ii) to every function $f \in L^{1}(S)$ there is a function $g \in L^{1}(S)$ such that $|g| \leq|f|$ and

$$
\int|E f(R x)|^{p} d \mu(x) \leq C_{p} \frac{\mathcal{C}_{\alpha, R}(\mu)}{R^{\alpha}} \int_{B(0,2 R)}|E g(y)|^{p} H(y) d y
$$

for $p \geq 1$, where $C_{p}$ is a constant that only depends on $p$.

Proof. We let $\phi$ be a Schwartz function on $\mathbb{R}^{3}$ such that $|\phi| \geq 1$ on $S$ and $\operatorname{supp} \widehat{\phi} \subset$ $B(0,1)$, and define the function $g$ on $S$ by $g=f / \phi$. Then $|g| \leq|f|$ and

$$
\int|E f(R x)|^{p} d \mu(x)=\int|\widehat{\phi} * \widehat{g d \sigma}(R x)|^{p} d \mu(x)=\int|\widehat{\phi} *(E g)(R x)|^{p} d \mu(x) .
$$

By Hölder's inequality, it follows that

$$
\begin{aligned}
\int|E f(R x)|^{p} d \mu(x) & \leq\|\widehat{\phi}\|_{L^{1}}^{p-1} \int|E g|^{p} *|\widehat{\phi}|(R x) d \mu(x) \\
& =\|\widehat{\phi}\|_{L^{1}}^{p-1} \iint|E g(y)|^{p}|\widehat{\phi}(R x-y)| d y d \mu(x) .
\end{aligned}
$$

Interchanging the order of integration, we arrive at the inequality

$$
\int|E f(R x)|^{p} d \mu(x) \leq\|\widehat{\phi}\|_{L^{1}}^{p-1}\|\widehat{\phi}\|_{L^{\infty}} \mathcal{C}_{\alpha, R}(\mu) R^{-\alpha} \int|E g(y)|^{p} H(y) d y,
$$

where $H(y)=\|\widehat{\phi}\|_{L^{\infty}}^{-1} \mathcal{C}_{\alpha, R}(\mu)^{-1} R^{\alpha} \int|\widehat{\phi}(R x-y)| d \mu(x)$. Since $\widehat{\phi}$ as $\mu$ is supported in $B(0,1)$, it follows that $H$ is supported in $B(0,1+R) \subset B(0,2 R)$, and hence

$$
\int|E f(R x)|^{p} d \mu(x) \leq\|\widehat{\phi}\|_{L^{1}}^{p-1}\|\widehat{\phi}\|_{L^{\infty}} \mathcal{C}_{\alpha, R}(\mu) R^{-\alpha} \int_{B(0,2 R)}|E g(y)|^{p} H(y) d y .
$$


It remains to show that $H$ is a weight of dimension $\alpha$ and $A_{\alpha}(H) \leq|B(0,1)|$. Clearly,

$$
H(y)=\|\widehat{\phi}\|_{L^{\infty}}^{-1} \mathcal{C}_{\alpha, R}(\mu)^{-1} R^{\alpha} \int_{B\left(R^{-1} y, R^{-1}\right)}|\widehat{\phi}(R x-y)| d \mu(x) \leq 1
$$

for all $y$, where we have used the fact that $\mu\left(B\left(R^{-1} y, R^{-1}\right)\right) \leq \mathcal{C}_{\alpha, R}(\mu) R^{-\alpha}$. Also,

$$
\begin{aligned}
\int_{B\left(x_{0}, r\right)} H(y) d y & =\|\widehat{\phi}\|_{L^{\infty}}^{-1} \mathcal{C}_{\alpha, R}(\mu)^{-1} R^{\alpha} \iint \chi_{B\left(x_{0}, r\right)}(y)|\widehat{\phi}(R x-y)| d \mu(x) d y \\
& =\|\widehat{\phi}\|_{L^{\infty}}^{-1} \mathcal{C}_{\alpha, R}(\mu)^{-1} R^{\alpha} \int|\widehat{\phi}(u)| \int \chi_{B\left(x_{0}, r\right)}(R x-u) d \mu(x) d u \\
& =\|\widehat{\phi}\|_{L^{\infty}}^{-1} \mathcal{C}_{\alpha, R}(\mu)^{-1} R^{\alpha} \int|\widehat{\phi}(u)| \mu\left(B\left(\left(u+x_{0}\right) R^{-1}, r R^{-1}\right)\right) d u \\
& \leq\|\widehat{\phi}\|_{L^{\infty}}^{-1}\|\widehat{\phi}\|_{L^{1}} r^{\alpha}
\end{aligned}
$$

provided $r \geq 1$. Thus $A_{\alpha}(H) \leq\|\widehat{\phi}\|_{L^{\infty}}^{-1}\|\widehat{\phi}\|_{L^{1}} \leq|B(0,1)|$.

\section{Proofs of the Corollaries}

We are now in position to prove our global restriction estimates.

Proof of Corollary 2.1, Let $3 / 2 \leq \alpha \leq 3$, and $H$ be a weight of dimension $\alpha$ with $A_{\alpha}(H) \leq 1$. We combine parts (i) and (ii) of Theorem 5.1 as

$$
\|\mathcal{R} f\|_{L^{p_{0}(S)}} \lesssim R^{\epsilon}\|f\|_{L^{s}\left(\chi_{B(0, R)} H d x\right)}
$$

with the understanding that $p_{0}=4(4 \alpha+3) /(10 \alpha+3)$ and $s^{\prime}=2(4 \alpha+3) /(2 \alpha+3)$ if $3 / 2 \leq \alpha<5 / 2$, and $13 / 9 \leq p_{0}<13 /(2+2 \alpha)$ and $s^{\prime}=13 / 4$ if $5 / 2 \leq \alpha \leq 3$. We note that in both cases $1<s \leq p_{0} \leq 2$.

Following 15, we would like to upgrade (12) to become valid for all functions $f \in L^{s}\left(\chi_{V} H d x\right)$ whenever $V$ is a union of a sparse family of balls. This means $V=\cup_{l=1}^{N} B\left(x_{l}, R\right)$ with $\left|x_{l}-x_{m}\right| \geq(R N)^{C}$ if $l \neq m$, where $C$ is a suitably large constant.

For $f \in L^{s}\left(\chi_{V} H d x\right)$ and $1 \leq l \leq N$, we let $f_{l}$ be the restriction of $f$ to $B\left(x_{l}, R\right)$, and we define the function $g_{l} \in L^{s}\left(\chi_{B(0, R)} H d x\right)$ by $g_{l}(x)=f_{l}\left(x+x_{l}\right)$. Then

$$
\mathcal{R} f_{l}(\xi)=\widehat{f_{l} H}(\xi)=e^{-2 \pi i \xi \cdot x_{l}}\left(g_{l} H\left(\cdot+x_{l}\right) \widehat{)}(\xi)\right.
$$

for all $\xi \in S$. Since $1<p_{0} \leq 2$ and the balls $B\left(x_{l}, R\right)$ are sparse, Lemma 3.2 of [15] as reformulated in [4] (see inequality (11) on page 1289 of [4]) now tells us that

$$
\|\mathcal{R} f\|_{L^{p_{0}(S)}}=\left\|\sum_{l=1}^{N} \mathcal{R} f_{l}\right\|_{L^{p_{0}(S)}} \lesssim\left(\sum_{l=1}^{N} \|\left(g_{l} H\left(\cdot+x_{l}\right) \hat{)} \|_{L^{p_{0}(S)}}^{p_{0}}\right)^{1 / p_{0}} .\right.
$$

Since $A_{\alpha}\left(H\left(\cdot+x_{l}\right)\right)=A_{\alpha}(H)$, (12) gives

$$
\|\left(g_{l} H\left(\cdot+x_{l}\right) \widehat{)}\left\|_{L^{p_{0}(S)}} \lesssim R^{\epsilon}\right\| g_{l} \|_{L^{s}\left(\chi_{B(0, R)} H\left(\cdot+x_{l}\right) d x\right)} .\right.
$$


But $\left\|g_{l}\right\|_{L^{s}\left(\chi_{B(0, R)} H\left(\cdot+x_{l}\right) d x\right)}=\|f\|_{L^{s}\left(\chi_{B\left(x_{l}, R\right)} H d x\right)}$, so

$$
\begin{aligned}
\|\mathcal{R} f\|_{L^{p_{0}}(S)} & \lesssim R^{\epsilon}\left(\sum_{l=1}^{N}\|f\|_{L^{s}\left(\chi_{B\left(x_{l}, R\right)} H d x\right)}^{p_{0}}\right)^{1 / p_{0}} \\
& \leq R^{\epsilon}\left(\sum_{l=1}^{N}\|f\|_{L^{s}\left(\chi_{B\left(x_{l}, R\right)} H d x\right)}^{s}\right)^{1 / s} \\
& =R^{\epsilon}\|f\|_{L^{s}\left(\chi_{V} H d x\right)},
\end{aligned}
$$

where we have used the fact that $p_{0} \geq s$.

The next step is to upgrade (12) to become valid for all $f \in L^{s}\left(\chi_{E} H d x\right)$ whenever $E$ is a finite union of $c$-cubes. For this we need the following lemma.

Lemma 6-A (Tao [15]). Suppose $E$ is the union of $c$-cubes and $0<\delta<1$. Then there are $O\left(\delta^{-1}|E|^{\delta}\right)$ sets $V_{k}$ that cover $E$ such that each $V_{k}$ is a union of a sparse collections of balls of radius $O\left(|E|^{C^{1 / \delta}}\right)$.

Given a function $f \in L^{s}\left(\chi_{E} H d x\right)$, then writing $f=\sum_{k} f_{k}$ with $f_{k}$ supported in $V_{k}$ and using Minkowski's inequality, we see that

$$
\|\mathcal{R} f\|_{L^{p_{0}(S)}} \lesssim \delta^{-1}|E|^{\delta}|E|^{\epsilon C^{1 / \delta}}\|f\|_{L^{s}\left(\chi_{E} H d x\right)} .
$$

Taking $\delta \sim 1 / \log (1 / \epsilon)$, this becomes

$$
\|\mathcal{R} f\|_{L^{p_{0}(S)}} \lesssim|E|^{C / \log (1 / \epsilon)}\|f\|_{L^{s}\left(\chi_{E} H d x\right)}
$$

provided $A_{\alpha}(H) \lesssim 1$.

We now take $H$ to be the characteristic function of $2 \Omega_{a, b}$, where $2 \Omega_{a, b}$ is the same as $\Omega_{a, b}$ but with the cylinder $\mathbb{R} \times[-1,1]^{2}$ replaced by $\mathbb{R} \times[-2,2]^{2}$, and proceed as in [4]. As we mentioned before, the argument in [4] is based on [15].

Suppose $1 \leq r<s$. Let $\tilde{E}$ be a subset of $2 \Omega_{a, b}$ which is a (possibly infinite) union of $c$-cubes, where $c$ is a constant that will be determined later, and let $f \in$ $L^{1}\left(2 \Omega_{a, b}\right) \cap L^{r}\left(2 \Omega_{a, b}\right)$ be a function that is constant on each of the $c$-cubes of $\tilde{E}$, vanishes on $2 \Omega_{a, b} \backslash \tilde{E}$, and satisfies $\|f\|_{L^{r}} \leq 1$. For $k \in \mathbb{Z}$, we set $E_{k}=$ $\left\{2^{-k-1} \leq|f|<2^{-k}\right\}$ and $f_{k}=\chi_{E_{k}} f$. Then each $E_{k}$ is a finite union of $c$-cubes, and $2^{-k r}\left|E_{k}\right| \lesssim 1$ for all $k$. We note that, since $\left|E_{k}\right| \geq c^{3}$, the last inequality implies that the set of $k$ for which $E_{k} \neq \emptyset$ is bounded from below by a constant that depends on $c$ and $r$. Applying (13) with $H=\chi_{2 \Omega_{a, b}}, f=f_{k}$, and $E=E_{k}$, we get

$$
\begin{aligned}
& \left\|\mathcal{R} f_{k}\right\|_{L^{p_{0}}(S)} \lesssim\left|E_{k}\right|^{C / \log (1 / \epsilon)}\left\|f_{k}\right\|_{L^{s}\left(2 \Omega_{a, b}\right)} \\
& \quad \lesssim 2^{-k}\left|E_{k}\right|^{C / \log (1 / \epsilon)}\left|E_{k}\right|^{1 / s} \lesssim 2^{-k^{k r C / \log (1 / \epsilon)} 2^{k r / s}} .
\end{aligned}
$$

Summing over $k$, we arrive at

$$
\|\mathcal{R} f\|_{L^{p_{0}(S)}} \lesssim 1
$$

provided

$$
\frac{C}{\log (1 / \epsilon)}+\frac{1}{s}<\frac{1}{r}
$$

For $\tau \in[-c / 2, c / 2]^{3}$, we now let $\mathcal{L}_{\tau}$ be the intersection of the lattice $c \mathbb{Z}^{3}+\tau$ with $\Omega_{a, b}$. We suppose $\left\{\lambda_{n}\right\}$ is a sequence in $l^{1}\left(\mathcal{L}_{\tau}\right) \cap l^{r}\left(\mathcal{L}_{\tau}\right)$, and let $\chi_{n}$ be the 
characteristic function of $[-c / 2, c / 2]^{3}+n$. If $f=\sum_{n} \lambda_{n} \chi_{n}$, then (14) tells us that

$$
\|\mathcal{R} f\|_{L^{p_{0}(S)}} \lesssim\left(\sum_{n}\left|\lambda_{n}\right|^{r}\right)^{1 / r}
$$

But $\widehat{f}(\xi)=\widehat{\chi_{0}}(\xi) \sum_{n} \lambda_{n} e^{-2 \pi i \xi \cdot n}$, so, choosing $c$ small enough for $\left|\widehat{\chi_{0}}\right|$ to be positive on $S$, we get

$$
\left(\int\left|\sum_{n \in \mathcal{L}_{\tau}} \lambda_{n} e^{-2 \pi i \xi \cdot n}\right|^{p_{0}} d \sigma(\xi)\right)^{1 / p_{0}} \lesssim\left(\sum_{n \in \mathcal{L}_{\tau}}\left|\lambda_{n}\right|^{r}\right)^{1 / r} .
$$

Averaging over $\tau$ and letting $p$ be the exponent conjugate to $r$, we get the estimate in part (i) of the corollary if $3 / 2 \leq \alpha<5 / 2$, and the estimate in part (ii) if $5 / 2 \leq \alpha \leq 3$.

When $\alpha=3$ and $a=b=1$, the estimate in part (ii) of the corollary becomes

$$
\|E f\|_{L^{p}\left(\mathbb{R}^{3}\right)}=\|E f\|_{L^{p}\left(\Omega_{1,1}\right)} \lesssim\|f\|_{L^{p_{0}^{\prime}(S)}}
$$

whenever $p>13 / 4$ and $1 \leq p_{0}<13 / 8$. Interpolating this with the trivial estimate $\|E f\|_{L^{\infty}\left(\mathbb{R}^{3}\right)} \leq\|f\|_{L^{1}(S)}$, we prove part (iii) of the corollary.

For the proof of Corollary 3.1 we will also need the following lemma from [19] that connects the $\mathcal{C}_{\alpha, R}(\mu)$ of Lemma 5.1 to the $\alpha$-dimensional energy of $\mu$.

Lemma 6-B (Wolff [19]). Let $\mu \in M\left(\mathbb{R}^{3}\right)$ be a positive measure with support in $B(0,1), 0<\alpha<3$, and $R \geq 1$. Then we can decompose $\mu$ as a sum of $O(1+\log R)$ measures $\mu_{j}$ so that for each $j$,

$$
\left\|\mu_{j}\right\| \mathcal{C}_{\alpha, R}\left(\mu_{j}\right) \lesssim I_{\alpha}(\mu)
$$

with an implicit constant that depends only on $\alpha$.

Proof of Corollary 3.1. As in the proof of Theorem 5.1, we let

$$
\bar{p}= \begin{cases}2(4 \alpha+3) /(2 \alpha+3) & \text { if } \frac{3}{2} \leq \alpha<\frac{5}{2} \\ 13 / 4 & \text { if } \frac{5}{2} \leq \alpha \leq 3\end{cases}
$$

Writing $\mu=\sum_{j} \mu_{j}$ as in Lemma 6-B we see by Hölder's inequality that

$$
\begin{aligned}
\int|E f(R x)| d \mu(x) & =\sum_{j} \int|E f(R x)| d \mu_{j}(x) \\
& \leq \sum_{j}\left\|\mu_{j}\right\|^{1-(1 / \bar{p})}\left(\int|E f(R x)|^{\bar{p}} d \mu_{j}(x)\right)^{1 / \bar{p}} \\
& \leq\|\mu\|^{1-(2 / \bar{p})} \sum_{j}\left(\left\|\mu_{j}\right\| \int|E f(R x)|^{\bar{p}} d \mu_{j}(x)\right)^{1 / \bar{p}}
\end{aligned}
$$

for all $f \in L^{1}(S)$, where we have used the fact that $\bar{p}>2$. But by Lemma 5.1 and Lemma 6-B, for each such $f$ there is a function $g$ with $|g| \leq|f|$ such that

$$
\begin{aligned}
\left\|\mu_{j}\right\| \int|E f(R x)|^{\bar{p}} d \mu_{j}(x) & \lesssim\left\|\mu_{j}\right\| \mathcal{C}_{\alpha, R}\left(\mu_{j}\right) R^{-\alpha} \int_{B(0,2 R)}|E g(y)|^{\bar{p}} H(y) d y \\
& \lesssim I_{\alpha}(\mu) R^{-\alpha} \int_{B(0,2 R)}|E g(y)|^{\bar{p}} H(y) d y
\end{aligned}
$$


Summing over $j$, we get

$$
\begin{aligned}
& \int|E f(R x)| d \mu(x) \\
& \quad \lesssim(1+\log R)\|\mu\|^{1-(2 / \bar{p})} I_{\alpha}(\mu)^{1 / \bar{p}} R^{-\alpha / \bar{p}}\left(\int_{B(0,2 R)}|E g(y)|^{\bar{p}} H(y) d y\right)^{1 / \bar{p}} .
\end{aligned}
$$

Since supp $\mu \subset B(0,1)$, we have $\|\mu\|^{2} \lesssim I_{\alpha}(\mu)$, and the above estimate becomes

$$
\int|E f(R x)| d \mu(x) \lesssim R^{\epsilon} I_{\alpha}(\mu)^{1 / 2} R^{-\alpha / \bar{p}}\left(\int_{B(0,2 R)}|E g(y)|^{\bar{p}} H(y) d y\right)^{1 / \bar{p}} .
$$

By part (i) of Lemma [5.1 we know that $A_{\alpha}(H) \lesssim 1$, so Theorem 5.1 (in its dual form) tells us that

$$
\left(\int_{B(0,2 R)}|E g(y)|^{\bar{p}} H(y) d y\right)^{1 / \bar{p}} \lesssim R^{\epsilon}\|g\|_{L^{p_{0}^{\prime}(S)}} .
$$

Thus

$$
\left|\int \widehat{\mu}(R \xi) f(\xi) d \sigma(\xi)\right| \lesssim R^{2 \epsilon} \frac{\sqrt{I_{\alpha}(\mu)}}{R^{\alpha / \bar{p}}}\|f\|_{L^{p_{0}^{\prime}(S)}}
$$

for all $f \in L^{p_{0}^{\prime}}(S)$ and $R \geq 1$. By duality, (15) proves parts (i) and (iii) of Corollary 3.1 .

If we use part (ii) of Theorem 1.1 instead of Theorem [5.1, and follow the same steps as in the proof of (15), we arrive at the inequality

$$
\left|\int \widehat{\mu}(R \xi) f(\xi) d \sigma(\xi)\right| \lesssim R^{2 \epsilon} \frac{\sqrt{I_{\alpha}(\mu)}}{R^{\alpha / 3}} R^{\frac{1}{12}\left(\alpha-\frac{3}{2}\right)}\|f\|_{L^{2}(S)}
$$

for all $f \in L^{2}(S)$ and $R \geq 1$. Inserting $\overline{\widehat{\mu}(R \xi)}$ for $f(\xi)$, the inequality becomes

$$
\left(\int|\widehat{\mu}(R \xi)|^{2} d \sigma(\xi)\right)^{1 / 2} \lesssim R^{2 \epsilon} \frac{\sqrt{I_{\alpha}(\mu)}}{R^{(\alpha / 4)+(1 / 8)}},
$$

which is part (ii) of Corollary 3.1 ,

We now move to prove our result on exponential sums.

Proof of Corollary 4.1. Let $\bar{p}$ and $\bar{\gamma}$ be as in the proof of Theorem 5.1. Since $\mathcal{C}_{\alpha, R}(\mu) \leq \mathcal{C}_{\alpha}(\mu)$ for all $R \geq 1$, parts (i) and (iii) of Theorem 1.1 together with Lemma 5.1 tell us that

$$
\int|E f(R x)|^{\bar{p}} d \mu(x) \lesssim \frac{R^{\epsilon}}{R^{\alpha}} \mathcal{C}_{\alpha}(\mu)\|f\|_{L^{2}(S)}^{\bar{\gamma}}\|f\|_{L^{\infty}(S)}^{\bar{p}-\bar{\gamma}}
$$

for all $f \in L^{\infty}(S)$. If $F$ is an $L^{\infty}$ function on the $1 / R$-neighborhood of $S$, then the above estimate implies that

$$
\int|\widehat{F}(R x)|^{\bar{p}} d \mu(x) \lesssim \mathcal{C}_{\alpha}(\mu) \frac{R^{\epsilon} R^{\bar{\gamma} / 2}}{R^{\alpha+\bar{p}}}\|F\|_{L^{2}}^{\bar{\gamma}}\|F\|_{L^{\infty}}^{\bar{p}-\bar{\gamma}}
$$


Proving this is a standard argument (e.g., see Proposition 4.3 of [18]). For example, if $S$ is the unit sphere, then

$$
\begin{aligned}
\widehat{F}(R x) & =\int_{1-\frac{1}{R} \leq|\xi| \leq 1+\frac{1}{R}} e^{-2 \pi i R x \cdot \xi} F(\xi) d \xi \\
& =\int_{1-\frac{1}{R}}^{1+\frac{1}{R}} \int_{S} e^{-2 \pi i R x \cdot r \theta} F(r \theta) d \sigma(\theta) r^{2} d r \\
& =\int_{1-\frac{1}{R}}^{1+\frac{1}{R}} E(F(r \cdot))(r R x) r^{2} d r
\end{aligned}
$$

so that

$$
\begin{aligned}
& \|\widehat{F}(R \cdot)\|_{L^{\bar{p}}(\mu)} \leq \int_{1-\frac{1}{R}}^{1+\frac{1}{R}}\|E(F(r \cdot))(r R \cdot)\|_{L^{\bar{p}}(\mu)} r^{2} d r \\
& \lesssim \mathcal{C}_{\alpha}(\mu)^{\frac{1}{\bar{p}}} \frac{R^{\frac{\epsilon}{\bar{p}}}}{R^{\frac{\alpha}{\bar{p}}}} \int_{1-\frac{1}{R}}^{1+\frac{1}{R}}\|F(r \cdot)\|_{L^{2}(S)}^{\frac{\bar{\gamma}}{\bar{p}}}\|F(r \cdot)\|_{L^{\infty}(S)}^{1-\frac{\bar{\gamma}}{\bar{p}}} r^{2} d r \\
& \lesssim \mathcal{C}_{\alpha}(\mu)^{\frac{1}{p}} \frac{R^{\frac{\epsilon}{\bar{p}}}}{R^{\frac{\alpha}{\bar{p}}}}\|F\|_{L^{\infty}}^{1-\frac{\bar{\gamma}}{p}} \frac{R^{\frac{\bar{\gamma}}{2 \bar{p}}}}{R}\left(\int_{1-\frac{1}{R}}^{1+\frac{1}{R}}\|F(r \cdot)\|_{L^{2}(S)}^{2} r^{2} d r\right)^{\frac{\bar{\gamma}}{2 \bar{p}}} \\
& =\mathcal{C}_{\alpha}(\mu)^{\frac{1}{\bar{p}}} \frac{R^{\frac{\epsilon}{\bar{p}}+\frac{\bar{\gamma}}{2 \bar{p}}}}{R^{\frac{\alpha}{\bar{p}}+1}}\|F\|_{L^{2}}^{\frac{\bar{\gamma}}{\bar{p}}}\|F\|_{L^{\infty}}^{1-\frac{\bar{\gamma}}{\bar{p}}},
\end{aligned}
$$

where we have used Hölder's inequality and the fact that $\bar{\gamma}<2 \bar{p}$.

Now suppose $w_{1}, \ldots, w_{N} \in S$ are such that $\left|w_{l}-w_{l^{\prime}}\right| \sim R^{-1}$. Let $\phi$ be a $C_{0}^{\infty}$ function on $\mathbb{R}^{3}$ with the property that $|\widehat{\phi}| \geq 1$ on the unit ball. Then

$$
\begin{aligned}
& \int\left|\sum_{l=1}^{N} a_{l} e^{-2 \pi i R w_{l} \cdot x}\right|^{\bar{p}} d \mu(x) \leq \int\left|\sum_{l=1}^{N} a_{l} e^{-2 \pi i w_{l} \cdot(R x)} \widehat{\phi_{R^{-1}}}(R x)\right|^{\bar{p}} d \mu(x) \\
& =\int\left|\sum_{l=1}^{N} \widehat{\psi}_{l}(R x)\right|^{\bar{p}} d \mu(x),
\end{aligned}
$$

where $\widehat{\psi_{l}}(y)=a_{l} e^{-2 \pi i w_{l} \cdot y} \widehat{\phi_{R^{-1}}}(y)$, i.e. $\psi_{l}(\xi)=a_{l} \phi_{R^{-1}}\left(\xi-w_{l}\right)=a_{l} R^{3} \phi\left(R\left(\xi-w_{l}\right)\right)$. Applying (16) with $F=\sum_{l=1}^{N} \psi_{l}$, we get

$$
\int\left|\sum_{l=1}^{N} a_{l} e^{-2 \pi i w_{l} \cdot x}\right|^{\bar{p}} d \mu(x) \lesssim \mathcal{C}_{\alpha}(\mu) \frac{R^{\epsilon} R^{\bar{\gamma} / 2}}{R^{\alpha+\bar{p}}}\|F\|_{L^{2}}^{\bar{\gamma}}\|F\|_{L^{\infty}}^{\bar{p}-\bar{\gamma}} .
$$

Since $\phi$ is compactly supported and the $w_{l}$ are $R^{-1}$-separated, we have

$$
\|F\|_{L^{2}}^{2} \lesssim R^{3} \sum_{l=1}^{N}\left|a_{l}\right|^{2} \quad \text { and } \quad\|F\|_{L^{\infty}} \lesssim R^{3} \max _{l}\left|a_{l}\right| .
$$

Thus

$$
\int\left|\sum_{l=1}^{N} a_{l} e^{-2 \pi i w_{l} \cdot x}\right|^{\bar{p}} d \mu(x) \lesssim \mathcal{C}_{\alpha}(\mu) \frac{R^{\epsilon} R^{2 \bar{p}}}{R^{\alpha+\bar{\gamma}}}\left(\sum_{l=1}^{N}\left|a_{l}\right|^{2}\right)^{\bar{\gamma} / 2}\left(\max _{l}\left|a_{l}\right|\right)^{\bar{p}-\bar{\gamma}} .
$$




\section{The WAVE PACKET DECOMPOSITION}

The wave packet decomposition is an important tool for studying the restriction problem. It translates the geometric condition (having strictly positive second fundamental form) imposed on the surface $S$ into a way of writing a function $f \in L^{2}(S)$ as a sum of simpler functions which are are almost orthogonal to each other and whose Fourier transforms are essentially supported on tubes. This idea was originated by Bourgain in 2] and was further developed by several authors (see [20], [16], 17], and [8]). Our presentation of this topic is tightly based on [8]. More precisely, we follow the proof of Proposition 2.6 in [8], but we organize the arguments and state the results in a slightly different manner.

Proposition 7.1. Suppose $\Theta$ is a closed ball in $\mathbb{R}^{n}$ of radius $\rho \leq 1, \delta>0$, and $\{D\}$ is a countable collection of closed balls in $\mathbb{R}^{n}$ of radius $\rho^{-1-\delta}$ satisfying

$$
1 \leq \sum_{D} \chi_{(3 / 4) D^{0}} \leq C
$$

for some constant $C$. Also, suppose that $\Phi \in C^{L}((4 / 3) \Theta)$ satisfies $\left|\nabla^{l} \Phi\right| \leq C_{L} \rho^{-l}$ for $0 \leq l \leq L$ and some constant $C_{L}, N>n$ is a positive integer, and $L \geq$ $n(4+\bar{\delta}+2 / \delta)+N(1+2 / \delta)$. Then to every function $f \in L^{2}\left(\mathbb{R}^{n}\right)$ with $\operatorname{supp} f \subset \Theta$ there is a sequence $\left\{f_{D}\right\}$ in $L^{2}\left(\mathbb{R}^{n}\right)$ with the following properties.

(i) Each $f_{D}$ is supported in $(4 / 3) \Theta, f=\sum_{D} f_{D}$ in $L^{1}((4 / 3) \Theta)$, and

$$
\sum_{D} \int\left|f_{D}\right|^{2} d \omega \leq\|f\|_{L^{2}(\Theta)}^{2} .
$$

(ii) We have

$$
\sum_{D: z \notin D}\left|\widehat{\Phi f_{D}}(z)\right| \lesssim \rho^{N}\|f\|_{L^{1}(\Theta)}
$$

for all $z \in \mathbb{R}^{n}$, where the implicit constant depends only on $L, C_{L}, C$, and $n$.

(iii) If $D_{1}$ and $D_{2}$ are disjoint, then

$$
\left|\int \Phi f_{D_{1}} \overline{f_{D_{2}}} d \omega\right| \lesssim \rho^{N}\|f\|_{L^{1}(\Theta)}^{2},
$$

where the implicit constant depends only on $L, C_{L}$, and $n$.

Proof. We start by letting $\psi_{\Theta} \in C^{\infty}\left(\mathbb{R}^{n}\right)$ be such that $0 \leq \psi_{\Theta} \leq 1, \psi_{\Theta}=1$ on $(5 / 4) \Theta, \psi_{\Theta}$ has support in $(4 / 3) \Theta$, and $\left|\nabla^{l} \psi_{\Theta}\right| \leq c_{l} \rho^{-l}$ for all $l$, where the $c_{l}$ are constants that depend only on $l$ and $n$. We also let $\Psi_{\Theta}=\Phi \psi_{\Theta}$. Clearly,

$$
\left|\nabla^{l} \Psi_{\Theta}\right| \lesssim \rho^{-l}
$$

for $0 \leq l \leq L$, and

$$
\left|\widehat{\Psi_{\Theta}}(z)\right| \lesssim \frac{|\Theta|}{(1+\rho|z|)^{L}}
$$

for all $z \in \mathbb{R}^{n}$, where the implicit constants depend only on $L, C_{L}$, and $n$.

We then let $\left\{\phi_{D}\right\}$ be a partition of unity of $\mathbb{R}^{n}$ subordinate to the open cover $\left\{(3 / 4) D^{0}\right\}$, and define the functions $f_{D}$ by

$$
f_{D}=\psi_{\Theta}\left(\varphi_{D} * f\right)
$$

where $\varphi_{D}$ is the inverse Fourier transform of $\phi_{D}$; in other words, $\varphi_{D}$ is given by $\varphi_{D}(\omega)=\widehat{\phi_{D}}(-\omega)$. 
(i) We clearly have $\operatorname{supp} f_{D} \subset(4 / 3) \Theta$ for all $D$. Since $\sum_{D} \phi_{D}=1$, it follows that $\sum_{D} \phi_{D} \widehat{f}$ converges to $\widehat{f}$ in $L^{2}\left(\mathbb{R}^{n}\right)$, so $\sum_{D} \varphi_{D} * f$ converges to $f$ in $L^{2}\left(\mathbb{R}^{n}\right)$ (by Plancherel), and so

$$
\sum_{D} f_{D}=\sum_{D} \psi_{\Theta}\left(\varphi_{D} * f\right)=\psi_{\Theta} \sum_{D} \varphi_{D} * f
$$

converges to $\psi_{\Theta} f=f$ in $L^{1}\left(\mathbb{R}^{n}\right)$ (by Cauchy-Schwarz). Also,

$$
\begin{gathered}
\sum_{D} \int\left|f_{D}\right|^{2} d \omega \leq \sum_{D} \int\left|\varphi_{D} * f\right|^{2} d \omega=\sum_{D} \int\left|\phi_{D}\right|^{2}|\widehat{f}|^{2} d z \\
\leq \int\left(\sum_{D}\left|\phi_{D}\right|\right)^{2}|\widehat{f}|^{2} d z=\int|\widehat{f}|^{2} d z=\int|f|^{2} d \omega
\end{gathered}
$$

where we have used Plancherel's theorem.

(ii) We have

$$
\widehat{\Phi f_{D}}=\widehat{\Psi_{\Theta}} *\left(\phi_{D} \widehat{f}\right)
$$

so

$$
\begin{aligned}
\left|\widehat{\Phi f_{D}}(z)\right| & =\left|\int \widehat{\Psi_{\Theta}}(z-y) \phi_{D}(y) \widehat{f}(y) d y\right| \\
& \lesssim \int_{\operatorname{supp} \phi_{D}} \frac{|\Theta|}{(1+\rho|z-y|)^{L}}\left|\phi_{D}(y)\right|\|f\|_{L^{1}(\Theta)} d y \\
& \leq \frac{\|f\|_{L^{1}(\Theta)}|\Theta|\left|\operatorname{supp} \phi_{D}\right|}{\left(1+\rho \operatorname{dist}\left(z, \operatorname{supp} \phi_{D}\right)\right)^{L}}
\end{aligned}
$$

for all $z \in \mathbb{R}^{n}$. Since $|\Theta| \sim \rho^{n}$ and $\operatorname{supp} \phi_{D}$ is contained in (3/4)D (which is a ball of radius $\left.(3 / 4) \rho^{-1-\delta}\right)$ ), it follows that

$$
\left|\widehat{\Phi f_{D}}(z)\right| \lesssim \frac{\|f\|_{L^{1}(\Theta)} \rho^{-n \delta}}{\left(1+\rho \operatorname{dist}\left(z, \operatorname{supp} \phi_{D}\right)\right)^{L}}
$$

for all $z \in \mathbb{R}^{n}$. If $z \notin D$, then $\operatorname{dist}\left(z, \operatorname{supp} \phi_{D}\right)>\rho^{-1-\delta} / 4$, and it follows that

$$
\begin{aligned}
\left|\widehat{\Phi f_{D}}(z)\right| & \lesssim \frac{\|f\|_{L^{1}(\Theta)} \rho^{-n \delta}}{\left(\rho+\rho \operatorname{dist}\left(z, \operatorname{supp} \phi_{D}\right)\right)^{N}\left(1+\rho \rho^{-1-\delta} / 4\right)^{L-N}} \\
& \leq \frac{4^{L-N}\|f\|_{L^{1}(\Theta)} \rho^{-n \delta-N+\delta(L-N)}}{\left(1+\operatorname{dist}\left(z, \operatorname{supp} \phi_{D}\right)\right)^{N}} \\
& \leq \frac{4^{L}\|f\|_{L^{1}(\Theta)} \rho^{N}}{\left(1+\operatorname{dist}\left(z, \operatorname{supp} \phi_{D}\right)\right)^{N}}
\end{aligned}
$$

provided $L \geq n+N+2 N / \delta$, where the implicit constants depend only on $L, C_{L}$, and $n$. Letting

$$
\mathcal{D}_{j}=\left\{D: 2^{j} \rho^{-1-\delta}<\operatorname{dist}\left(z, \operatorname{supp} \phi_{D}\right) \leq 2^{j+1} \rho^{-1-\delta}\right\}
$$


for $j=-2,-1, \ldots$, we then see that

$$
\begin{aligned}
\sum_{D: z \notin D}\left|\widehat{\Phi f_{D}}(z)\right| & \lesssim \rho^{N}\|f\|_{L^{1}(\Theta)} \sum_{D: z \notin D}\left(1+\operatorname{dist}\left(z, \operatorname{supp} \phi_{D}\right)\right)^{-N} \\
& \leq \rho^{N}\|f\|_{L^{1}(\Theta)} \sum_{j=-2}^{\infty} \sum_{D \in \mathcal{D}_{j}}\left(1+\operatorname{dist}\left(z, \operatorname{supp} \phi_{D}\right)\right)^{-N} \\
& \leq \rho^{N}\|f\|_{L^{1}(\Theta)} \sum_{j=-2}^{\infty} \sum_{D \in \mathcal{D}_{j}}\left(1+2^{j} \rho^{-1-\delta}\right)^{-N} \\
& \lesssim \rho^{N}\|f\|_{L^{1}(\Theta)},
\end{aligned}
$$

where the implicit constants depend only on $L, C_{L}, C$, and $n$.

(iii) Suppose $D_{1}$ and $D_{2}$ are disjoint. Then, by Plancherel's theorem,

$$
\int \Phi f_{D_{1}} \overline{f_{D_{2}}} d \omega=\int \Phi f_{D_{1}} \overline{\psi_{\Theta}} \overline{\left(\varphi_{D_{2}} * f\right)} d \omega=\int\left(\Phi \overline{\psi_{\Theta}} f_{D_{1}} \hat{)} \overline{\phi_{D_{2}} \widehat{f}} d z .\right.
$$

The estimates in part (ii) that lead to (17) apply to $\left(\Phi \overline{\psi_{\Theta}} f_{D_{1}} \hat{)}\right.$ (since $\Phi \overline{\psi_{\Theta}}$ has the same smoothness and decay properties as $\Phi$ ), so (by (17))

$$
\mid\left(\Phi \overline{\psi_{\Theta}} f_{D_{1}} \hat{)}(z) \mid \lesssim \rho^{N^{\prime}}\|f\|_{L^{1}(\Theta)}\right.
$$

for all $x \in D_{1}^{c}$ (and hence for all $x \in D_{2}$ ) provided $L \geq n+N^{\prime}+2 N^{\prime} / \delta$, and so

$$
\begin{aligned}
& \left|\int \Phi f_{D_{1}} \overline{f_{D_{2}}} d \omega\right| \lesssim \rho^{N^{\prime}}\|f\|_{L^{1}(\Theta)} \int_{\operatorname{supp} \phi_{D_{2}}}\left|\phi_{D_{2}}\right|\|f\|_{L^{1}(\Theta)} d z \\
& \leq \rho^{N^{\prime}}\|f\|_{L^{1}(\Theta)}^{2}\left|D_{2}\right| \sim \rho^{N^{\prime}}\|f\|_{L^{1}(\Theta)}^{2} \rho^{-n(1+\delta)} \lesssim \rho^{N}\|f\|_{L^{1}(\Theta)}^{2}
\end{aligned}
$$

provided $N^{\prime} \geq N+n(1+\delta)$.

We now consider a $C^{L}$ function $h: \Omega \rightarrow \mathbb{R}$ defined on some open set $\Omega \subset \mathbb{R}^{n}$, and we assume the following bounds on the first and second order partial derivatives of $h$ :

$$
\begin{cases}\frac{1}{2} \leq \partial_{i}^{2} h(\omega) \leq \frac{3}{2} & \text { for } i=1, \ldots, n, \\ \left|\partial_{i} \partial_{j} h(\omega)\right| \leq \frac{1}{4(n-1)} & \text { if } i \neq j, \\ |\nabla h(\omega)| \leq \frac{7}{4} & \end{cases}
$$

for all $\omega \in \Omega$.

We let $B$ be a closed ball in $\Omega$ of center $\omega_{0}$ and radius $r \leq 1 / 12, \theta$ be the graph of $h$ over $B$, and $3 \theta$ be the graph of $h$ over $3 B$. We are going to show that in an appropriate orthonormal system of coordinates, $\theta$ is contained in the graph of a $C^{L}$ function $h_{0}$, defined in a ball of radius $\sim r$, such that both $h_{0}$ and $\nabla h_{0}$ vanish at the center of the ball, and the graph of $h_{0}$ is contained in $3 \theta$.

Let $\mathcal{T}_{\left(\omega_{0}, h\left(\omega_{0}\right)\right)} \theta$ be the tangent plane to $\theta$ at $\left(\omega_{0}, h\left(\omega_{0}\right)\right)$. If $\omega$ is a point on the boundary of $B$ and $(\Delta \omega, \Delta h)=\left(\omega-\omega_{0}, h(\omega)-h\left(\omega_{0}\right)\right)$, then Pythagoras' theorem shows that the projection of $(\Delta \omega, \Delta h)$ onto $\mathcal{T}_{\left(\omega_{0}, h\left(\omega_{0}\right)\right)} \theta$ has length

$$
\rho_{0}=\left(r^{2}+(\Delta h)^{2}-\frac{\left(\Delta h-\nabla h\left(\omega_{0}\right) \cdot \Delta \omega\right)^{2}}{1+\left|\nabla h\left(\omega_{0}\right)\right|^{2}}\right)^{1 / 2} .
$$


By (18), $|\Delta h| \leq(7 / 4) r$, so $\rho_{0} \leq(\sqrt{65} / 4) r$. On the other hand, Taylor's theorem and (18) tell us that

$$
\left|\Delta h-\nabla h\left(\omega_{0}\right) \cdot \Delta \omega\right| \leq \frac{1}{2}\left(\frac{3}{2}|\Delta \omega|^{2}+\frac{1}{4}|\Delta \omega|^{2}\right)<r^{2},
$$

so $\rho_{0}>r \sqrt{1-r^{2}}$, and so $\sqrt{15} r<4 \rho_{0} \leq \sqrt{65} r$ (because $r<3 r \leq 1 / 4$ ). This shows that if we let $\theta^{\prime}$ and $(3 \theta)^{\prime}$ be the projections of $\theta$ and $3 \theta$, respectively, onto $\mathcal{T}_{\left(\omega_{0}, h\left(\omega_{0}\right)\right)} \theta$, and if we dilate $\theta^{\prime}$ around $\left(\omega_{0}, h\left(\omega_{0}\right)\right)$ by a factor of $4 / 3$, then the resulting set will be contained in $(3 \theta)^{\prime}$. More precisely, letting $\Theta$ be the ball in $\mathcal{T}_{\left(\omega_{0}, h\left(\omega_{0}\right)\right)} \theta$ of center $\left(\omega_{0}, h\left(\omega_{0}\right)\right)$ and radius $\rho=(4 / \sqrt{15}) \rho_{0}$ (note that $r<\rho \leq$ $\sqrt{13 / 3} r)$, we have

$$
\theta^{\prime} \subset \Theta \subset(4 / 3) \Theta \subset(3 \theta)^{\prime}
$$

(because $(4 / 3) \rho \leq(4 / 3)(\sqrt{13 / 3}) r<(\sqrt{15} / 4)(3 r))$. Therefore, in an appropriate orthonormal system of coordinates, $\theta$ is contained in the graph of a $C^{L}$ function $h_{0}:(4 / 3) \Theta \rightarrow \mathbb{R}$ such that both $h_{0}$ and $\nabla h_{0}$ vanish at the center of $\Theta$, and the graph of $h_{0}$ is contained in $3 \theta$. For the rest of this section, all the implicit constants will depend on the $C^{L}$ norm of $h_{0}$.

Let $S_{0}$ be the graph of $h_{0}$. We know that $\theta \subset S_{0} \subset 3 \theta$. Let $f$ be a function in $L^{2}\left(S_{0}\right)$ with support in $\theta$. We would now like to obtain the wave packet decomposition of $f$. We start by replacing the function $f(\omega)$ in Proposition 7.1 by the function $f\left(\omega, h_{0}(\omega)\right) J(\omega)$, where $J(\omega)=\sqrt{1+\left|\nabla h_{0}(\omega)\right|^{2}}$. To each member of the collection $\{D\}$, we associate a tube $T \subset \mathbb{R}^{n+1}$ defined by $T=D \times \mathbb{R}$. We alert the reader that this definition of $T$ is in the new system of coordinates that comes with the function $h_{0}$; in the original system of coordinates the tube $T$ is perpendicular to the tangent plane $\mathcal{T}_{\left(\omega_{0}, h\left(\omega_{0}\right)\right)} \theta$. We then define the function $f_{T} \in L^{2}\left(S_{0}\right)$ by

$$
f_{T}\left(\omega, h_{0}(\omega)\right)=\frac{\left(f\left(\cdot, h_{0}(\cdot)\right) J\right)_{D}(\omega)}{J(\omega)} .
$$

Next, we apply parts (i) and (ii) of Proposition 7.1 with $\Phi(\omega)=e^{-2 \pi i z_{n+1} h_{0}(\omega)}$. Since $\left|\partial_{j} h_{0}\right| \lesssim \rho$ for $j=1, \ldots, n$, we need $\left|z_{n+1}\right| \leq \rho^{-2}$ in order to satisfy the requirement $\left|\nabla^{l} \Phi\right| \leq C_{L} \rho^{-l}$. To free the condition $\left|z_{n+1}\right| \leq \rho^{-2}$ from depending on the choice of the orthonormal coordinates, we require $\left|\left(z, z_{n+1}\right)\right| \leq \rho^{-2} \sim r^{-2}$.

We know that $f\left(\cdot, h_{0}(\cdot)\right) J=\sum_{D}\left(f\left(\cdot, h_{0}(\cdot)\right) J\right)_{D}$ in $L^{1}((4 / 3) \Theta)$, so $f=\sum_{T} f_{T}$ in $L^{1}\left(S_{0}\right)$, and

$$
\begin{aligned}
\sum_{T} \int\left|f_{T}\right|^{2} d \sigma & =\sum_{D} \int \frac{\left|\left(f\left(\cdot, h_{0}(\cdot)\right) J\right)_{D}(\omega)\right|^{2}}{J(\omega)} d \omega \\
& \leq \sum_{D} \int\left|\left(f\left(\cdot, h_{0}(\cdot)\right) J\right)_{D}(\omega)\right|^{2} d \omega \\
& \leq \int \mid\left(\left.f\left(\omega, h_{0}(\omega)\right) J(\omega)\right|^{2} d \omega\right. \\
& \lesssim \int|f|^{2} d \sigma .
\end{aligned}
$$

We also have

$$
E g\left(z, z_{n+1}\right)=\left(\Phi g\left(\cdot, h_{0}(\cdot)\right) \widehat{J)}(z)\right.
$$


for all $g \in L^{1}\left(S_{0}\right)$, so

$$
\begin{aligned}
& \sum_{T:\left(z, z_{n+1}\right) \notin T}\left|E f_{T}\left(z, z_{n+1}\right)\right|=\sum_{D: z \notin D}\left(\Phi\left(f\left(\cdot, h_{0}(\cdot)\right) J\right)_{D} \hat{)}\right. \\
& \lesssim \rho^{N}\left\|f\left(\cdot, h_{0}(\cdot)\right) J\right\|_{L^{1}(\Theta)}=\rho^{N}\|f\|_{L^{1}(\theta)}=\rho^{N}\|f\|_{L^{1}\left(S_{0}\right)} .
\end{aligned}
$$

The functions $f_{T}$ are almost orthogonal in $L^{2}\left(S_{0}\right)$. To see this, we apply part (iii) of Proposition 7.1 with $\Phi(\omega)=1 / J(\omega)$ to get

$$
\begin{gathered}
\int f_{T_{1}} \overline{f_{T_{2}}} d \sigma=\int \Phi(\omega)\left(f\left(\cdot, h_{0}(\cdot)\right) J\right)_{D_{1}}(\omega) \overline{\left(f\left(\cdot, h_{0}(\cdot)\right) J\right)_{D_{2}}(\omega)} d \omega \\
\lesssim \rho^{N}\left\|f\left(\cdot, h_{0}(\cdot)\right) J\right\|_{L^{1}(\Theta)}^{2}=\rho^{N}\|f\|_{L^{1}(\theta)}^{2}=\rho^{N}\|f\|_{L^{1}\left(S_{0}\right)}^{2} .
\end{gathered}
$$

We summarize the above discussion in the following proposition, which is a reformulation of Proposition 2.6 in [8].

Proposition 7.2. Suppose $S$ is a compact $C^{L}$ surface in $R^{n+1}$ given as the graph of a function $h$ that satisfies (18), $\delta>0, N>n / 2$ is a positive integer, and

$$
L \geq n\left(4+2 \delta+\frac{1}{\delta}\right)+(2 N)\left(1+\frac{1}{\delta}\right) .
$$

Let $\theta$ be a cap on $S$ of center $\xi_{0}$ and radius $r=R^{-1 / 2} \leq 1 / 12$, and $v(\theta)$ be the unit normal vector of $S$ at $\xi_{0}$.

Then there is a countable collection $\tilde{\mathbb{T}}(\theta)=\{T\}$ of finitely overlapping tubes in $\mathbb{R}^{n+1}$ of radius $R^{(1 / 2)+\delta}$, which are parallel to $v(\theta)$, such that the following holds. To every function $f \in L^{2}(S)$ with supp $f \subset \theta$ there is a sequence $\left\{f_{T}\right\}$ in $L^{2}(S)$ with the following properties.

(i) Each $f_{T}$ is supported in $3 \theta, f=\sum_{T \in \tilde{\mathbb{T}}(\theta)} f_{T}$ in $L^{1}(S)$, and

$$
\sum_{T \in \tilde{\mathbb{T}}(\theta)} \int\left|f_{T}\right|^{2} d \sigma \lesssim\|f\|_{L^{2}(S)}^{2},
$$

where the implicit constant depends only on the $C^{1}$ norm of $h$.

(ii) We have

$$
\sum_{T \in \tilde{\mathbb{T}}(\theta): x \notin T}\left|E f_{T}(x)\right| \lesssim R^{-N}\|f\|_{L^{1}(S)}
$$

for all $x \in \mathbb{R}^{n+1}$ with $|x| \leq R$, where the implicit constant depends only on $L, N$, $n$, and the $C^{L}$ norm of $h$.

(iii) If $T_{1}, T_{2} \in \tilde{\mathbb{T}}(\theta)$ are disjoint, then

$$
\left|\int f_{T_{1}} \overline{f_{T_{2}}} d \sigma\right| \lesssim R^{-N}\|f\|_{L^{1}(S)}^{2}
$$

where the implicit constant depends only on $L, N$, n, and the $C^{L}$ norm of $h$. (iv) Let $\mathbb{T}(\theta)=\{T \in \tilde{\mathbb{T}}(\theta): T \cap B(0, R) \neq \emptyset\}$. Then

$$
\left|E f(x)-\sum_{T \in \mathbb{T}(\theta)} E f_{T}(x)\right| \lesssim R^{-N}\|f\|_{L^{1}(\theta)}
$$

for all $x \in B(0, R)$. 
We note that in applying Proposition 7.1 to get Proposition 7.2 we have replaced $\delta$ by $2 \delta$ and $N$ by $2 N$. We also note that part (iv) of Proposition 7.2 is an immediate consequence of part(ii).

For each $T \in \tilde{\mathbb{T}}(\theta)$, the function $f_{T}$ is called a wave packet. The equality $f=$ $\sum_{T \in \tilde{\mathbb{T}}(\theta)} f_{T}$ (which holds in $L^{1}(S)$ ) is called the wave packet decomposition of $f$. The functions that we shall be dealing with for the rest of the paper are defined on a surface $S \subset \mathbb{R}^{3}$. This means that we shall be using Proposition 7.1 in $\mathbb{R}^{2}$, and Proposition 7.2 in $\mathbb{R}^{3}$.

\section{Guth's POlYnomial PARTitioning METHOD}

In this section, and for the rest of the paper, we make the following assumption on the surface $S$.

Assumption 8.1. The surface $S$ is the graph of a function $h: B^{2}(0,1) \rightarrow \mathbb{R}$ that satisfies the following conditions.

(i) There is an integer $L \geq 3$ such that $h \in C^{L}\left(B^{2}(0,1)\right)$.

(ii) We have $h(0)=\nabla h(0)=0$.

(iii) For all $\omega \in B^{2}(0,1)$, both eigenvalues of the Hessian $\partial^{2} h(\omega)$ lie in the open interval $(3 / 4,5 / 4)$.

(iv) We have $\left\|\nabla^{l} h\right\|_{L^{\infty}\left(B^{2}(0,1)\right)}<10^{-9}$ for $3 \leq l \leq L$.

Once we have proved that to every $\epsilon>0$ there is a positive integer $L_{\epsilon}$ such that Theorem 1.1 holds for all surfaces that satisfy (i)-(iv) with $L \geq L_{\epsilon}$, the result for general $C^{\infty}$ compact surfaces with strictly positive second fundamental form would follow by a standard parabolic scaling argument. We refer the reader to the last paragraph of Subsection 2.3 in [8] for a very nice outline of this argument.

The information we have about the eigenvalues of $\partial^{2} h$ give the following bounds on the second order partial derivatives of $h$ :

$$
\begin{cases}\frac{1}{2}<\partial_{i}^{2} h(\omega)<\frac{3}{2} & \text { for } i=1,2, \\ \left|\partial_{i} \partial_{j} h(\omega)\right|<\frac{1}{4} & \text { if } i \neq j\end{cases}
$$

for all $\omega \in B^{2}(0,1)$. These bounds tell us that $\left\|\partial^{2} h(\omega)\right\|<7 / 4$ for all $\omega \in B^{2}(0,1)$, where $\left\|\partial^{2} h(\omega)\right\|$ is the operator norm of $\partial^{2} h(\omega)$. Condition (ii) then implies that

$$
|\nabla h(\omega)|<(7 / 4)|\omega| \quad \forall \omega \in B^{2}(0,1) .
$$

Thus $h$ satisfies (18) (with $n=2$ ) on some open set $\Omega$ that contains the closed unit ball $B^{2}(0,1)$. Thus Proposition 7.2 applies to functions on $S$. For this reason, in this and the next four sections, we will let $N>1$ and $\delta$ be, respectively, a positive integer and a positive number that satisfy the standing hypothesis

$$
L \geq 2\left(4+2 \delta+\frac{1}{\delta}\right)+(2 N)\left(1+\frac{1}{\delta}\right) .
$$

At the end of the argument, it will be clear that to find the positive integer $L_{\epsilon}$ that was mentioned above (following the statement of Assumption 8.1), we need to take $N$ to be a large absolute constant, say $N=1000$, and $\delta$ to be small relative to $\epsilon$, say $\delta=\epsilon^{2}$.

Let $P$ be a polynomial in $n$ real variables of degree $D$, and $Z(P)$ be the zero set of $P$. A connected component of $\mathbb{R}^{n} \backslash Z(P)$ is called a cell. If $\left\{O_{i}\right\}$ are all the cells of $\mathbb{R}^{n} \backslash Z(P)$, then $|\{i\}| \leq C_{n} D^{n}$ for some constant $C_{n}$ that only depends on the 
dimension $n$. (For a proof of this bound on the number of cells, we refer the reader to Milnor [13.) A line in $\mathbb{R}^{n}$, however, can intersect at most $D+1$ cells. This relationship between lines and polynomials lies at the heart of what is now referred to in incidence geometry and harmonic analysis as the polynomial method.

The polynomial method helped resolve a number of longstanding combinatorial problems in incidence geometry concerning the intersection patterns of lines in Euclidean space as well as in vector spaces over finite fields. In harmonic analysis, the combinatorial issues concern the intersection pattern of tubes with a fixed radius $\rho$. A tube can enter much more than $D+1$ of the cells $\left\{O_{i}\right\}$. To go around this difficulty, Guth defined in 8 , the cell-wall $W$ as the $\rho$-neighborhood of $Z(P)$ and considered the sets $O_{i}^{\prime}=O_{i} \backslash W$. Guth then observed that if a tube enters $O_{i}^{\prime}$, then its core line will enter $O_{i}$, so the tube can enter at most $D+1$ of the modified cells $\left\{O_{i}^{\prime}\right\}$.

Another property of polynomials that lies at the heart of the polynomial method (when working in Euclidean space rather than in vector spaces over finite fields) is that given a degree $D$ and a non-negative integrable function $F$, one can use the topology of $\mathbb{R}^{n}$ to find a polynomial $P$ of degree at most $D$ such that the integrals $\int_{O_{i}} F d x$ are essentially equal.

Theorem 8-A (Corollary 1.7 in [8]). Let $F$ be a non-negative function in $L^{1}\left(\mathbb{R}^{n}\right)$. Then to every $D$ there is a non-zero polynomial $P$ of degree at most $D$ such that $P$ is a product of non-singular polynomial $3^{3}, \mathbb{R}^{n} \backslash Z(P)$ is a disjoint union of $\sim D^{n}$ cells $O_{i}$, and the integrals $\int_{O_{i}} F(x) d x$ agree up to a factor of 2 .

Given a function $f \in L^{1}(S)$, our goal is to estimate the $L^{p}(H d x)$ norm of $E f$ over the ball $B_{R}$ in $\mathbb{R}^{3}$ of center 0 and radius $R$. We shall think of $B_{R}$ as lying in physical space and of the surface $S$ as lying in frequency space. In physical space, $B_{R}$ inherits from $\mathbb{R}^{3}$ a partition into cells $O_{i}^{\prime}$ and a cell-wall $W$ coming from a polynomial $P$, as described above. The only condition we impose on $P$ for now is that it is a product of non-singular polynomials in three real variables; we will not use Theorem 8 -A until we arrive at the proof of Theorem 9.1. In frequency space, we cover $S$ by a collection $\{\theta\}$ of finitely-overlapping caps each of radius $R^{-1 / 2}$, and we write $f=\sum_{\theta} f_{\theta}$ with $f_{\theta}$ supported in $\theta$ and such that $\left(\operatorname{supp} f_{\theta}\right) \cap\left(\operatorname{supp} f_{\theta^{\prime}}\right)=\emptyset$ if $\theta \neq \theta^{\prime}$. Applying Proposition 7.2 of the previous section to each $f_{\theta}$, we obtain a wave packet decomposition of $f$ :

$$
f=\sum_{\theta} \sum_{T \in \tilde{\mathbb{T}}(\theta)}\left(f_{\theta}\right)_{T}
$$

with the equality holding in $L^{1}(S)$.

We point out that the covering $\{\theta\}$ of $S$ by $R^{-1 / 2}$-caps will be fixed throughout the argument, so, in order to simplify the notation, we write $f_{T}$ for $\left(f_{\theta}\right)_{T}$.

Going back to physical space, applying part (iv) of Proposition 7.2 to each $f_{\theta}$ and summing shows that

$$
E f(x)=\sum_{T \in \mathbb{T}} E f_{T}(x)+O\left(R^{-N}\|f\|_{L^{1}(S)}\right)
$$

for all $x \in B_{R}$, where

$$
\mathbb{T}=\cup_{\theta} \mathbb{T}(\theta) .
$$

\footnotetext{
${ }^{3}$ Recall that a polynomial $Q$ is non-singular if $\nabla Q(x) \neq 0$ for all $x \in Z(Q)$.
} 
Recall from Proposition 7.2 that each $T \in \mathbb{T}$ is a tube of radius $R^{(1 / 2)+\delta}$, so the cell-wall is

$$
W=N_{R^{(1 / 2)+\delta}} Z(P) .
$$

If $\mathbb{T}_{i}$ is a subset of $\mathbb{T}$, we set

$$
f_{i}=\sum_{T \in \mathbb{T}_{i}} f_{T}
$$

We shall often denote a function on $S$ which is supported in a cap $\tau$ by $f_{\tau}$. In this case, we shall write $f_{\tau, i}$ for $\left(f_{\tau}\right)_{i}$ and $f_{\tau, T}$ for $\left(f_{\tau}\right)_{T}$, so that

$$
f_{\tau, i}=\sum_{T \in \mathbb{T}_{i}} f_{\tau, T}
$$

If we happen to have $f=\sum_{\tau} f_{\tau}$, then 4

$$
f_{i}=\sum_{T \in \mathbb{T}_{i}} f_{T}=\sum_{T \in \mathbb{T}_{i}} \sum_{\tau} f_{\tau, T}=\sum_{\tau} \sum_{T \in \mathbb{T}_{i}} f_{\tau, T}=\sum_{\tau} f_{\tau, i} .
$$

Since the $f_{\tau, T}$ are almost orthogonal (by part (iii) of Proposition 7.2), one expects $\sum_{T \in \mathbb{T}_{i}}\left\|f_{\tau, T}\right\|_{L^{2}(S)}^{2}$ to be smaller than $\left\|f_{\tau}\right\|_{L^{2}(S)}^{2}$. The next lemma makes this precise.

Lemma 8-A (Lemma 2.7 in [8]). Suppose $\left\{\mathbb{T}_{i}\right\}_{i \in I}$ is a family of subsets of $\mathbb{T}, k$ is a positive integer, and $\tau \subset S$. If each tube $T \in \cup_{i \in I} \mathbb{T}_{i}$ belongs to at most $k$ of the subsets $\left\{\mathbb{T}_{i}\right\}_{i \in I}$, then

$$
\sum_{i \in I} \int_{3 \theta}\left|f_{\tau, i}\right|^{2} d \sigma(\xi) \lesssim k \int_{10 \theta}\left|f_{\tau}\right|^{2} d \sigma(\xi)
$$

for all $\theta$. Also,

$$
\sum_{i \in I} \int_{S}\left|f_{\tau, i}\right|^{2} d \sigma(\xi) \lesssim k \int_{S}\left|f_{\tau}\right|^{2} d \sigma(\xi)
$$

We now fix a specific family $\left\{\mathbb{T}_{i}\right\}_{i \in I}$ of subsets of $\mathbb{T}$. We define

$$
\mathbb{T}_{i}=\left\{T \in \mathbb{T}: T \cap O_{i}^{\prime} \neq \emptyset\right\} .
$$

Recalling Guth's motivation for introducing the modified cells $O_{i}^{\prime}$, we know that a tube $T$ of radius $R^{(1 / 2)+\delta}$ can enter at most $D+1$ of these cells. So, a tube $T \in \mathbb{T}$ can belong to at most $D+1$ of the sets $\mathbb{T}_{i}$. For later reference, we state this fact in the following lemma.

Lemma 8-B (Lemma 3.2 in [8]). A tube $T \in \mathbb{T}$ can belong to at most $D+1$ of the sets $\mathbb{T}_{i}$.

The integral of $|E f|^{p} H$ on the cells $O_{i}^{\prime} \cap B_{R}$ will be controlled using induction. To control the integral of $|E f|^{p} H$ on $W \cap B_{R}$, we cover $B_{R}$ with $\sim R^{3 \delta}$ balls $B_{j}$ of radius $R^{1-\delta}$. If $B_{j} \cap W \neq \emptyset$, then the tubes of $\mathbb{T}$ will be separated into two groups: the tubes that are tangent to $Z(P)$ in $B_{j}$, and the tubes that are transverse to $Z(P)$ in $B_{j}$. Here are the details.

Let $Z_{0}(P)$ be the set of all non-singular points of $Z(P)$. We denote by $\mathbb{T}_{j, \text { tang }}$ the set of all $T \in \mathbb{T}$ satisfying

$$
\left\{\begin{array}{l}
T \cap W \cap B_{j} \neq \emptyset \\
\operatorname{Angle}\left(v(T), T_{z} Z(P)\right) \leq R^{-(1 / 2)+2 \delta} \forall z \in Z_{0}(P) \cap 2 B_{j} \cap 10 T,
\end{array}\right.
$$

\footnotetext{
${ }^{4}$ One can easily see from the proof of Proposition 7.1 that $(f+g)_{T}=f_{T}+g_{T}$.
} 
where $v(T)$ is the unit vector in the direction of the tube $T$, and by $\mathbb{T}_{j, \text { trans }}$ the set of all $T \in \mathbb{T}$ satisfying

$$
\left\{\begin{array}{l}
T \cap W \cap B_{j} \neq \emptyset \\
\exists z \in Z_{0}(P) \cap 2 B_{j} \cap 10 T \text { such that Angle }\left(v(T), T_{z} Z(P)\right)>R^{-(1 / 2)+2 \delta} .
\end{array}\right.
$$

Any tube $T \in \mathbb{T}$ that intersects $W \cap B_{j}$ lies in exactly one of $\mathbb{T}_{j \text {,tang }}$ and $\mathbb{T}_{j, \text { trans }}$. For a proof of this fact, we refer the reader to the paragraph immediately following Definitions 3.3 and 3.4 in [8]. More importantly, we have the following two remarkable results of $[8]$ :

Lemma 8-C (Lemma 3.5 in [8]). If $P$ has degree at most $D$, then a tube $T \in \mathbb{T}$ can belong to at most $\operatorname{Poly}(D)$ different sets $\mathbb{T}_{j, \text { trans }}$.

Lemma 8-D (Lemma 3.6 in [8]). If $P$ has degree at most $D$, then, for each $j$, the number of different $\theta$ such that $\mathbb{T}_{j, \text { tang }} \cap \mathbb{T}(\theta) \neq \emptyset$ is at most $D^{2} R^{(1 / 2)+O(\delta)}$.

We let

$$
f_{\tau, j, \text { tang }}=\sum_{T \in \mathbb{T}_{j, \text { tang }}} f_{\tau, T} \quad \text { and } \quad f_{j, \text { tang }}=\sum_{\tau} f_{\tau, j, \text { tang }}
$$

and

$$
f_{\tau, j, \text { trans }}=\sum_{T \in \mathbb{T}_{j, \text { trans }}} f_{\tau, T} \quad \text { and } \quad f_{j, \text { trans }}=\sum_{\tau} f_{\tau, j, \text { trans }} .
$$

Recall that $S$ is covered by $\sim K^{2}$ caps $\tau$ of diameter $1 / K$. If $I$ is any subset of these caps, we let

$$
f_{I, j, \text { trans }}=\sum_{\tau \in I} f_{\tau, j, \text { trans }} .
$$

The contribution to $\int_{B_{j} \cap W}|E f(x)|^{p} H(x) d x$ coming from the transverse tubes will be controlled by using induction to estimate

$$
\sum_{I} \int_{B_{j} \cap W}\left|E f_{I, j, \text { trans }}(x)\right|^{p} H(x) d x
$$

where the sum runs over all $I \subset\{\tau\}$. To control the contribution coming from the tangential tubes, we make a further definition.

We say that two caps $\tau_{1}$ and $\tau_{2}$ are non-adjacent if the distance between them is $\geq 1 / K$. We define

$$
\operatorname{Bil}_{P, \delta} E f_{j, \text { tang }}=\sum_{\tau_{1}, \tau_{2} \text { non-adjacent }}\left|E f_{\tau_{1}, j, \text { tang }}\right|^{1 / 2}\left|E f_{\tau_{2}, j, \text { tang }}\right|^{1 / 2} .
$$

We call the function $\mathrm{Bil}_{P, \delta} E f_{j \text {,tang }}$ the tangential part of $E f$ with respect to the polynomial $P$ and the parameter $\delta$. Of course, this function also depends on $R$, the ball $B_{j}$, and the decomposition $\{\tau\}$ of $S$, but $R, B_{j}$ and $\{\tau\}$ will often appear elsewhere in the estimates involving $\operatorname{Bil}_{P, \delta} E f_{j, \text { tang }}$, so to simplify the notation we only emphasize the dependence of this function on $P$ and $\delta$ (see the statement of Theorem 9.1 below).

It is not clear how controlling the tangential part of $E f$ on $B_{j} \cap W$ can lead to controlling the contribution to $\int_{B_{j} \cap W}|E f(x)|^{p} H(x) d x$ coming from the tangential tubes. One of the important ideas of $[8]$ is that controlling $\operatorname{Bil}_{P, \delta} E f_{j, \operatorname{tang}}$ on $B_{j} \cap W$ actually leads to controlling the contribution of the tangential tubes to the $L^{p}$ norm of the broad part of $E f$ on $B_{j} \cap W$. 
The definition of the broad part of $E f$ and its relation to $\operatorname{Bil}_{P, \delta} E f_{j, \operatorname{tang}}$ is the subject of our next section.

Guth estimated the $L^{p}(d x)$ norm of $\operatorname{Bil}_{P, \delta} E f_{j, \text { tang }}$ over $B_{j} \cap W$ by using Córdoba's $L^{4}$ argument (see Lemma 3.10 in $[8$, or Lemma 11-A below), and used this estimate to derive an estimate on the broad part of $E f$, before going back to $E f$ itself.

In the next section, we formulate a general theorem, Theorem 9.1 saying that if one has a favorable bound on the $L^{p}(H d x)$ norm of $\operatorname{Bil}_{P, \delta} E f_{j \text {,tang }}$ over $B_{j} \cap W$, then one gets a favorable estimate on the broad part of $E f$ over $B_{R}$.

In Section 11, we establish bounds on various $L^{p}(H d x)$ norms of $\operatorname{Bil}_{P, \delta} E f_{j, \text { tang }}$ over $B_{j} \cap W$. We then insert these bounds into Theorem 9.1 and arrive at our desired estimates on the $L^{p}(H d x)$ norm of the broad part of $E f$ on $B_{R}$.

\section{THE BROAD PART AND ITS INTERACTION WITH THE ZERO SET OF $P$}

We are now in good shape to present Guth's definition of the broad part of $E f$. We let $m$ and $K$ be constants, and we think of $K$ as being rather large. We consider a covering $\left\{B^{2}(\omega, r)\right\}$ of $B^{2}(0,1)$ such that the centers $\omega$ are $K^{-1}$-separated, and the radius $r$ satisfies the inequalities $1 / K \leq r \leq \sqrt{m} / K$. If a point $\omega_{0}$ belongs to $M$ of these balls, then the centers of the $M$ balls lie in $B^{2}\left(\omega_{0}, \sqrt{m} / K\right)$. Since the $\omega$ are $1 / K$-separated, it follows that $M \leq c m$ for some absolute constant $c$. Letting $\tau$ be the graph of $h$ over $B^{2}(\omega, r)$ (i.e., $\tau$ is a cap on $S$ of center $(\omega, h(\omega))$ and radius $r$ ), we obtain a covering of $S$ by caps $\tau$ such that each point of $S$ lies in at most $\mathrm{cm}$ different caps. We shall refer to $\{\tau\}$ as a decomposition of $S$ of multiplicity $m$. We write $f=\sum_{\tau} f_{\tau}$ with $\operatorname{supp} f_{\tau} \subset \tau$, but we do not insist that $\left(\operatorname{supp} f_{\tau}\right) \cap\left(\operatorname{supp} f_{\tau^{\prime}}\right)=\emptyset$ if $\tau \neq \tau^{\prime}$, as we did with the decomposition $f=\sum_{\theta} f_{\theta}$ above.

Suppose $0<\beta \leq 1$. We say the point $x \in \mathbb{R}^{3}$ is $\beta$-broad for $E f$ if

$$
\max _{\tau}\left|E f_{\tau}(x)\right|<\beta|E f(x)| .
$$

Since $|E f(x)| \lesssim K^{2} \max _{\tau}\left|E f_{\tau}(x)\right|$, a necessary condition for the existence of $\beta$ broad points is that $\beta \gtrsim K^{-2}$. Also, if $f=f_{\tau}$ for some $\tau$, then (since $\beta \leq 1$ ) no point of physical space can satisfy the above inequality, so another necessary condition for the existence of $\beta$-broad points is that $f$ is not supported in just one of the caps $\tau$.

We now define the $\beta$-broad part of $E f$ to be the function $\operatorname{Br}_{\beta} E f: \mathbb{R}^{3} \rightarrow[0, \infty)$ given by

$$
\operatorname{Br}_{\beta} E f(x)= \begin{cases}|E f(x)| & \text { if } x \text { is } \beta \text {-broad for } E f \\ 0 & \text { otherwise. }\end{cases}
$$

Clearly,

$$
|E f(x)|^{p} \leq \operatorname{Br}_{\beta} E f(x)^{p}+\frac{1}{\beta^{p}} \sum_{\tau}\left|E f_{\tau}(x)\right|^{p}
$$

for all $x \in \mathbb{R}^{3}$ and $p>0$. Guth's strategy in [8] is to estimate $\int_{B_{R}} \operatorname{Br}_{\beta} E f(x)^{p} d x$ (for appropriate $\beta$ ) by using polynomial partitioning and induction, and estimate $\int_{B_{R}}\left|E f_{\tau}(x)\right|^{p} d x$ by using parabolic scaling and induction.

Recall from (21) that $E f \sim \sum_{T \in \mathbb{T}} E f_{T}$ on $B_{R}$. Since $E f_{T}$ is essentially supported on the tube $T$, to estimate $\int_{O_{i}^{\prime} \cap B_{R}}|E f(x)|^{p} H(x) d x$, one may replace the function $f$ by the function $f_{i}=\sum_{T \in \mathbb{T}_{i}} f_{T}$. We remind the reader that

$$
\mathbb{T}_{i}=\left\{T \in \mathbb{T}: T \cap O_{i}^{\prime} \neq \emptyset\right\}
$$


The next lemma says that this is also true for the broad part of $E f$. We include the proof of this lemma, as well as the proof of Lemma 9-B below, because of their importance to the flow of the argument. Also, because of some minor differences between the statements here and the corresponding statements in [8]; for example, the form of the error terms, and the conditions on $K$ and $R$.

Lemma 9-A (Lemma 3.7 in [8]). Suppose $\epsilon, K>0, K^{-\epsilon} \leq \beta \leq 1$, and $R \geq C K^{\epsilon}$. If $x \in O_{i}^{\prime}$ and $C$ is sufficiently large, then

$$
\operatorname{Br}_{\beta} E f(x) \leq \operatorname{Br}_{2 \beta} E f_{i}(x)+O\left(R^{-N+1} \sum_{\tau}\left\|f_{\tau}\right\|_{L^{1}(S)}\right) .
$$

Proof ([8]). From (21), we know that

$$
E f_{\tau}(x)=\sum_{T \in \mathbb{T}} E f_{\tau, T}(x)+O\left(R^{-N}\left\|f_{\tau}\right\|_{L^{1}(S)}\right)
$$

The point $x$ is in $O_{i}^{\prime}$. If $x \in T$, then $T$ must intersect $O_{i}^{\prime}$, and it follows that $T \in \mathbb{T}_{i}$. If $x \notin T$ and $T \in \mathbb{T}(\theta)$, then part (ii) of Proposition 7.2 tells us that $\left|E f_{\tau, T}(x)\right| \lesssim R^{-N}\left\|f_{\tau}\right\|_{L^{1}(\theta)}$, so

$$
\sum_{T \notin \mathbb{T}_{i}}\left|E f_{\tau, T}(x)\right|=\sum_{\theta} \sum_{T \in \mathbb{T}(\theta) \backslash \mathbb{T}_{i}}\left|E f_{\tau, T}(x)\right| \lesssim \sum_{\theta} R^{-N}\left\|f_{\tau}\right\|_{L^{1}(\theta)}=R^{-N}\left\|f_{\tau}\right\|_{L^{1}(S)},
$$

and so

$$
E f_{\tau}(x)=\sum_{T \in \mathbb{T}_{i}} E f_{\tau, T}(x)+O\left(R^{-N}\left\|f_{\tau}\right\|_{L^{1}(S)}\right)=E f_{\tau, i}(x)+O\left(R^{-N}\left\|f_{\tau}\right\|_{L^{1}(S)}\right) .
$$

Summing over $\tau$ and using (22), we get

$$
E f(x)=E f_{i}(x)+O\left(R^{-N} \sum_{\tau}\left\|f_{\tau}\right\|_{L^{1}(S)}\right) .
$$

Since we can assume that $|E f(x)|>R^{-N+1} \sum_{\tau}\left\|f_{\tau}\right\|_{L^{1}(S)}$, it follows that

$$
\left|E f_{i}(x)\right|>\frac{1}{2} R^{-N+1} \sum_{\tau}\left\|f_{\tau}\right\|_{L^{1}(S)} .
$$

(We have $R \geq C K^{\epsilon} \geq C$, so for the last inequality we need $C^{\prime} / C \leq 1 / 2$, where $C^{\prime}$ is the implicit constant in the error term in the last equality.) We can also assume that $x$ is $\beta$-broad for $E f$. Under these assumptions, it remains to show that $x$ is $(2 \beta)$-broad for $E f_{i}$. In other words, we have to show that for each $\tau$,

$$
\left|E f_{\tau, i}(x)\right| \leq 2 \beta\left|E f_{i}(x)\right| .
$$

The equality before the last tells us that

$$
\left|E f_{\tau, i}(x)\right| \leq\left|E f_{\tau}(x)\right|+O\left(R^{-N}\left\|f_{\tau}\right\|_{L^{1}(S)}\right) \leq \beta|E f(x)|+O\left(R^{-N}\left\|f_{\tau}\right\|_{L^{1}(S)}\right) .
$$

The last equality then tells us that

$$
\left|E f_{\tau, i}(x)\right| \leq \beta\left|E f_{i}(x)\right|+O\left(R^{-N} \sum_{\tau}\left\|f_{\tau}\right\|_{L^{1}(S)}\right)
$$


(recall that $\beta \leq 1$ ). Thus

$$
\begin{aligned}
\left|E f_{\tau, i}(x)\right| & \leq \beta\left|E f_{i}(x)\right|+\frac{1}{R} O\left(R^{-N+1} \sum_{\tau}\left\|f_{\tau}\right\|_{L^{1}(S)}\right) \\
& \leq \beta\left|E f_{i}(x)\right|+\frac{K^{-\epsilon}}{C} O\left(R^{-N+1} \sum_{\tau}\left\|f_{\tau}\right\|_{L^{1}(S)}\right) \\
& \leq \beta\left|E f_{i}(x)\right|+\frac{K^{-\epsilon}}{C}\left(2 C^{\prime \prime}\left|E f_{i}(x)\right|\right) \\
& =2 \beta\left|E f_{i}(x)\right|
\end{aligned}
$$

provided $C$ is sufficiently large, where $C^{\prime \prime}$ is the implicit constant in the error term.

The next lemma connects the broad part of $E f, \operatorname{Br}_{\beta} E f$, to the tangential part of $E f, \operatorname{Bil}_{P, \delta} E f_{j, \text { tang. }}$. We remind the reader that $N$ and $\delta$ satisfy (20).

Lemma 9-B (Lemma 3.8 in [8]). Suppose $0<\epsilon \leq 2, K \geq \sqrt[98]{10}, K^{-\epsilon} \leq \beta \leq 1$, $\beta m \leq 10^{-5}$, and $R \geq C K^{\epsilon}$. If $x \in B_{j} \cap W$ and $C$ is sufficiently large, then

$$
\begin{aligned}
\operatorname{Br}_{\beta} E f(x) \leq & \frac{5}{4} \sum_{I} \operatorname{Br}_{2 \beta} E f_{I, j, \text { trans }}(x)+K^{100} \operatorname{Bil}_{P, \delta} E f_{j, \operatorname{tang}}(x) \\
& +O\left(R^{-N+1} \sum_{\tau}\left\|f_{\tau}\right\|_{L^{1}(S)}\right) .
\end{aligned}
$$

Proof ([8]). We can assume that $|E f(x)|>R^{-N+1} \sum_{\tau}\left\|f_{\tau}\right\|_{L^{1}(S)}$ and $x$ is $\beta$-broad for $E f$. Let $I$ be the set of the caps $\tau$ such that $\left|E f_{\tau, j, \text { tang }}(x)\right| \leq K^{-100}|E f(x)|$. We can also assume that $I^{c}$ does not contain two non-adjacent caps. Then $I^{c}$ consists of at most $10^{4} \mathrm{~m}$ caps. In fact, since the centers of the caps are $K^{-1}$-separated, and the radius of each cap is at most $\sqrt{m} K^{-1}$, we have $\left|I^{c}\right| \lesssim\left(\sqrt{m} K^{-1}\right)^{2} /\left(K^{-1}\right)^{2}=m$. Since $x$ is $\beta$-broad for $E f$, and $\beta m \leq 10^{-5}$, it follows that

$$
\sum_{\tau \in I^{c}}\left|E f_{\tau}(x)\right| \leq 10^{4} m \beta|E f(x)| \leq \frac{1}{10}|E f(x)|
$$

so that $|E f(x)| \leq(10 / 9)\left|E f_{I}(x)\right|$. If $x \in T$, then $T$ must intersect $B_{j} \cap W$, and it follows that $T$ belongs to $\mathbb{T}_{j, \text { trans }}$ or $\mathbb{T}_{j, \text { tang. }}$. If $x \notin T$ and $T \in \mathbb{T}(\theta)$, then $\left|E f_{\tau, T}(x)\right| \lesssim R^{-N}\left\|f_{\tau}\right\|_{L^{1}(\theta)}$, so

$$
\begin{aligned}
\sum_{T \notin \mathbb{T}_{j, \text { trans }} \cup \mathbb{T}_{j, \text { tang }}}\left|E f_{\tau, T}(x)\right| & =\sum_{\theta} \sum_{T \in \mathbb{T}(\theta) \backslash\left(\mathbb{T}_{j, \text { trans }} \cup \mathbb{T}_{j, \text { tang }}\right)}\left|E f_{\tau, T}(x)\right| \\
& \lesssim \sum_{\theta} R^{-N}\left\|f_{\tau}\right\|_{L^{1}(\theta)} \\
& \lesssim R^{-N}\left\|f_{\tau}\right\|_{L^{1}(S)},
\end{aligned}
$$

and so (recall that $T_{j, \operatorname{tang}} \cap T_{j, \text { trans }}=\emptyset$ )

$$
\begin{aligned}
E f_{\tau}(x) & =\sum_{T \in T_{j, \text { trans }}} E f_{\tau, T}(x)+\sum_{T \in T_{j, \text { tang }}} E f_{\tau, T}(x)+O\left(R^{-N}\left\|f_{\tau}\right\|_{L^{1}(S)}\right) \\
& =E f_{\tau, j, \text { trans }}(x)+E f_{\tau, j, \text { tang }}(x)+O\left(R^{-N}\left\|f_{\tau}\right\|_{L^{1}(S)}\right) .
\end{aligned}
$$


Summing over $\tau \in I$, we see that

$$
E f_{I}(x)=E f_{I, j, \operatorname{trans}}(x)+E f_{I, j, \text { tang }}(x)+O\left(R^{-N} \sum_{\tau}\left\|f_{\tau}\right\|_{L^{1}(S)}\right) .
$$

By the definition of $I$,

$$
\begin{aligned}
& \qquad\left|E f_{I, j, \text { tang }}(x)\right| \leq \sum_{\tau \in I}\left|E f_{\tau, j, \text { tang }}(x)\right| \leq \sum_{\tau \in I} K^{-100}|E f(x)| \leq K^{-98}|E f(x)|, \\
& \text { so } \\
& \frac{9}{10}|E f(x)| \leq\left|E f_{I}(x)\right| \leq\left|E f_{I, j, \text { trans }}(x)\right|+K^{-98}|E f(x)|+O\left(R^{-N} \sum_{\tau}\left\|f_{\tau}\right\|_{L^{1}(S)}\right) \text {, }
\end{aligned}
$$

and so

$$
\frac{4}{5}|E f(x)| \leq\left|E f_{I, j, \operatorname{trans}}(x)\right|+\frac{C^{\prime}}{R}\left(R^{-N+1} \sum_{\tau}\left\|f_{\tau}\right\|_{L^{1}(S)}\right)
$$

provided $K^{-98} \leq 10^{-1}$, where $C^{\prime}$ is the implicit constant in the error term of the inequality before the last.

It remains to prove that $x$ is $(2 \beta)$-broad for $E f_{I, j, \text { trans }}(x)$. Since $|E f(x)|>$ $R^{-N+1} \sum_{\tau}\left\|f_{\tau}\right\|_{L^{1}(S)}$, we see that

$$
\left|E f_{I, j, \text { trans }}(x)\right|>\frac{1}{2} R^{-N+1} \sum_{\tau}\left\|f_{\tau}\right\|_{L^{1}(S)} .
$$

(We have $R \geq C K^{\epsilon} \geq C$, so for the last inequality we need $C^{\prime} / C \leq 3 / 10$.) Also, for $\tau \in I,\left|E f_{\tau, j, \operatorname{tang}}(x)\right| \leq K^{-100}|E f(x)|$, so (23) tells us that

$$
\begin{aligned}
\left|E f_{\tau, j, \operatorname{trans}}(x)\right| & \leq\left|E f_{\tau}(x)\right|+\left|E f_{\tau, j, \operatorname{tang}}(x)\right|+O\left(R^{-N}\left\|f_{\tau}\right\|_{L^{1}(S)}\right) \\
& \leq\left(\beta+K^{-100}\right)|E f(x)|+O\left(R^{-N}\left\|f_{\tau}\right\|_{L^{1}(S)}\right),
\end{aligned}
$$

where we have also used the fact that $x$ is $\beta$-broad for $E f$. Since

$$
\frac{4}{5}|E f(x)| \leq\left|E f_{I, j, \operatorname{trans}}(x)\right|+\frac{C^{\prime}}{R}\left(R^{-N+1} \sum_{\tau}\left\|f_{\tau}\right\|_{L^{1}(S)}\right),
$$

$K^{-\epsilon} \leq \beta$, and $K^{-98} \leq 1 / 10$, we get

$$
\begin{aligned}
\left|E f_{\tau, j, \operatorname{trans}}(x)\right| & \leq \frac{5}{4}\left(\beta+\frac{\beta^{2 / \epsilon}}{10}\right)\left|E f_{I, j, \operatorname{trans}}(x)\right|+\frac{C^{\prime \prime}}{R}\left(R^{-N+1} \sum_{\tau}\left\|f_{\tau}\right\|_{L^{1}(S)}\right) \\
& \leq \frac{55}{40} \beta\left|E f_{I, j, \text { trans }}(x)\right|+\frac{2 C^{\prime \prime} K^{-\epsilon}}{C}\left|E f_{I, j, \text { trans }}(x)\right| \\
& \leq 2 \beta\left|E f_{I, j, \text { trans }}(x)\right|
\end{aligned}
$$

provided $2 C^{\prime \prime} / C \leq 25 / 40$. Thus $x$ is $(2 \beta)$-broad for $\left|E f_{I, j \text {,trans }}(x)\right|$, as desired.

We are now in position to state the main result of this section. For $(R, K, m, b) \in$ $[1, \infty)^{4}$, we let $\Lambda(R, K, m, b)$ be the set of all functions $f \in L^{1}(S)$ such that $f=$ $\sum_{\tau} f_{\tau}$ for some decomposition $\{\tau\}$ of $S$ of multiplicity $m$ with supp $f_{\tau} \subset \tau$ and

$$
\int_{B\left(\xi_{0}, R^{-1 / 2}\right) \cap S}\left|f_{\tau}(\xi)\right|^{2} d \sigma(\xi) \leq \frac{1}{R^{(b+1) / 2}}
$$

for all $\xi_{0} \in S$. Since $S$ can be covered by $\sim R$ of such balls $B\left(\xi_{0}, R^{-1 / 2}\right)$, (24) tells us that

$$
\int\left|f_{\tau}(\xi)\right|^{2} d \sigma(\xi) \lesssim \frac{1}{R^{(b-1) / 2}}
$$


During the proof of Theorem 1.1 in the last section of the paper, we are going to use two different values of $b$ depending on whether we allow the final estimate to involve both $\|f\|_{L^{2}(S)}$ and $\|f\|_{L^{\infty}(S)}$, or insist on only involving $\|f\|_{L^{2}(S)}$.

Remark 9.1. It is important to note that all constants in the next theorem are allowed to depend on $L$ and $\alpha$, but are uniform for all functions $h$ satisfying conditions (i)-(iv) of Assumption 8.1, and all weights $H$ of dimension $\alpha$. This fact will be crucial to the induction argument that we will later use (see Theorem 12.1) to move from estimates on the broad part of Ef to estimates on Ef itself.

Theorem 9.1. Let $3<p \leq 4, b \geq 1, \epsilon>0,0 \leq q_{1} \leq 1 \leq 2 q_{0}, 0<q_{2}<q_{0}$, and $H$ be a weight of dimension $\alpha$. Also, let $\delta=\epsilon^{2}, \delta_{\mathrm{deg}}=\epsilon^{4}$, and $\delta_{\mathrm{trans}}=\epsilon^{6}$.

Suppose that

$$
\begin{aligned}
& \int_{B_{j} \cap W} \operatorname{Bil}_{P, \delta} E f_{j, \text { tang }}(x)^{p} H(x) d x \\
& \quad \leq C_{\epsilon, K} R^{O(\delta)} R^{q_{2} \epsilon} A_{\alpha}(H)^{q_{1}}\left(\sum_{\tau}\left\|f_{\tau}\right\|_{L^{2}(S)}^{2}\right)^{(3 / 2)+\epsilon}
\end{aligned}
$$

whenever $R \geq C, K \geq 100, m \geq 1, f \in \Lambda(R, K, m, b), P$ is a polynomial of degree at most $D=R^{\delta_{\mathrm{deg}}}$, and $P$ is a product of non-singular polynomial on $\mathbb{R}^{3}$.

Then there is a constant $c_{0}$, which is independent of $q_{0}, q_{1}, b$, and $p$, such that if $\epsilon \leq \min \left[c_{0},(p-3) / 2\right]$, then there is a $K=K(\epsilon)$ such that

$$
\begin{aligned}
& \int_{B_{R}} \operatorname{Br}_{\beta} E f(x)^{p} H(x) d x \\
& \quad \leq C_{\epsilon} R^{q_{0} \epsilon} A_{\alpha}(H)^{q_{1}}\left(\sum_{\tau}\left\|f_{\tau}\right\|_{L^{2}(S)}^{2}\right)^{(3 / 2)+\epsilon} R^{\delta_{\text {trans }} \log \left(K^{\epsilon} \beta m\right)}
\end{aligned}
$$

for all $\beta \geq K^{-\epsilon}, m \geq 1, R \geq 1$, and $f \in \Lambda(R, K, m, b)$. Moreover, $\lim _{\epsilon \rightarrow 0} K(\epsilon)=\infty$.

Our proof of Theorem 9.1 which is the subject of the next section, follows to a large extent the proof of Theorem 3.1 in Guth's paper [8]. There are three main differences between our theorem and Guth's theorem. First, we are working in the weighted setting. Second, in [8], (24) is replaced by

$$
\int_{B\left(\xi_{0}, R^{-1 / 2}\right) \cap S}\left|f_{\tau}(\xi)\right|^{2} d \sigma(\xi) \leq \frac{1}{R} .
$$

Third, the conclusion of Theorem 9.1 is conditional on (26): if (26) holds, then (27) holds.

\section{Proof of TheOrem 9.1}

We alert the reader that in order to guarantee the independence of $c_{0}$ from $q_{0}$, $q_{1}, b$, and $p$, we will be careful to check that all the constants (implicit and explicit) that appear in this proof are independent of these parameters.

We may assume that the constant $C$ is large enough to satisfy the requirements of Lemmas $9-\mathrm{A}$ and $9-\mathrm{B}$. Lemma $9-\mathrm{B}$ also requires that $\beta m \leq 10^{-5}$. To meet this requirement, we set

$$
K=K(\epsilon)=e^{\epsilon^{-10}}
$$

and notice that

$$
R^{\delta_{\mathrm{trans}} \log \left(K^{\epsilon} \beta m\right)} \geq R^{\epsilon^{6} \log \left(K^{\epsilon} 10^{-6}\right)} \geq R^{\epsilon^{-4}}
$$


if $\beta m \geq 10^{-6}$. So can assume that $\beta m \leq 10^{-6}$. In fact,

$$
\begin{aligned}
\int_{B_{R}} H(x) d x & =\left(\int_{B_{R}} H(x) d x\right)^{q_{1}}\left(\int_{B_{R}} H(x) d x\right)^{1-q_{1}} \\
& \leq A_{\alpha}(H)^{q_{1}} R^{\alpha q_{1}}|B(0,1)|^{1-q_{1}} R^{3-3 q_{1}} \\
& \leq 5 A_{\alpha}(H)^{q_{1}} R^{3}
\end{aligned}
$$

(because $|B(0,1)| \leq 5,0 \leq q_{1} \leq 1$ and $\alpha \leq 3$ ) and

$$
\|f\|_{L^{2}(S)} \leq \sum_{\tau}\left\|f_{\tau}\right\|_{L^{2}(S)} \lesssim K\left(\sum_{\tau}\left\|f_{\tau}\right\|_{L^{2}(S)}^{2}\right)^{1 / 2}
$$

(because the cardinality of $\{\tau\}$ is $\sim K^{2}$ ), so

$$
\begin{aligned}
\int_{B_{R}} & \operatorname{Br}_{\beta} E f(x)^{p} H(x) d x \\
& \lesssim A_{\alpha}(H)^{q_{1}}\left(\sum_{\tau}\left\|f_{\tau}\right\|_{L^{2}(S)}^{2}\right)^{p / 2} R^{3} \\
& \lesssim A_{\alpha}(H)^{q_{1}}\left(\sum_{\tau}\left\|f_{\tau}\right\|_{L^{2}(S)}^{2}\right)^{\frac{3}{2}+\epsilon}\left(\sum_{\tau}\left\|f_{\tau}\right\|_{L^{2}(S)}^{2}\right)^{\frac{p-3}{2}-\epsilon} R^{3} \\
& \lesssim A_{\alpha}(H)^{q_{1}}\left(\sum_{\tau}\left\|f_{\tau}\right\|_{L^{2}(S)}^{2}\right)^{(3 / 2)+\epsilon} R^{\delta_{\text {trans }} \log \left(K^{\epsilon} \beta m\right)}
\end{aligned}
$$

provided $p>3+2 \epsilon, \beta m \geq 10^{-6}$, and $R^{3} \leq R^{\epsilon^{-4}}$, where we have used (25) and the fact that $(p-3) / 2-\epsilon \leq 1 / 2$.

The theorem will be proved by induction. We see from (28) (and (25)) that (27) holds for $R \leq C K^{\epsilon}$. So we assume that $R \geq C K^{\epsilon}$ and that the theorem is true for all radii in the interval $[1, R / 2]$. We also see from (28) that (27) holds if

$$
\sum_{\tau}\left\|f_{\tau}\right\|_{L^{2}(S)}^{2} \leq R^{-3\left(\frac{p-3}{2}-\epsilon\right)^{-1}},
$$

so we also assume that the theorem is true for all functions $g \in \Lambda(R, K, m, b)$ with $\sum_{\tau}\left\|g_{\tau}\right\|_{L^{2}(S)}^{2} \leq(1 / 2) \sum_{\tau}\left\|f_{\tau}\right\|_{L^{2}(S)}^{2}$.

In the discussion leading to the statement of Theorem 9.1, $P$ was a general polynomial on $\mathbb{R}^{3}$ that was only required to be a product of non-singular polynomials (see the paragraph following Theorem $8-\mathrm{A}$ ). Now we pick a specific polynomial $P$. We apply Theorem 8 -A with $n=3, F=\chi_{B_{R}}\left(\operatorname{Br}_{\beta} E f\right)^{p} H$, and $D=R^{\delta_{\operatorname{deg}}}$ to get a polynomial $P$ of degree at most $D$ such that $P$ is a product of non-singular polynomials, $\mathbb{R}^{3} \backslash Z(P)$ is a disjoint union of $\sim D^{3}$ cells $O_{i}$, and

$$
\int_{O_{i} \cap B_{R}} \operatorname{Br}_{\beta} E f(x)^{p} H(x) d x \sim D^{-3} \int_{B_{R}} \operatorname{Br}_{\beta} E f(x)^{p} H(x) d x .
$$

Let the modified cells $O_{i}^{\prime}$ and the cell-wall $W$ be as defined above, i.e. $W=$ $N_{R^{(1 / 2)+\delta}} Z(P)$ and $O_{i}^{\prime}=O_{i} \backslash W$. Then

$$
\begin{aligned}
& \int_{B_{R}} \operatorname{Br}_{\beta} E f(x)^{p} H(x) d x \\
& \quad=\sum_{i} \int_{B_{R} \cap O_{i}^{\prime}} \operatorname{Br}_{\beta} E f(x)^{p} H(x) d x+\int_{B_{R} \cap W} \operatorname{Br}_{\beta} E f(x)^{p} H(x) d x .
\end{aligned}
$$


10.1. The cellular case. Suppose the cellular term dominates. Then there are $\sim D^{3}$ different cells $O_{i}^{\prime}$ such that

$$
\int_{B_{R} \cap O_{i}^{\prime}} \operatorname{Br}_{\beta} E f(x)^{p} H(x) d x \sim D^{-3} \int_{B_{R}} \operatorname{Br}_{\beta} E f(x)^{p} H(x) d x .
$$

We write $f_{i}=\sum_{\tau} f_{\tau, i}$, as in (22), and use Lemma 8-A] (applied to a single subset $\mathbb{T}_{i} \subset \mathbb{T}$ ) to see that

$$
\int_{B\left(\xi_{0}, R^{-1 / 2}\right) \cap S}\left|f_{\tau, i}\right|^{2} d \sigma(\xi) \leq c \int_{B\left(\xi_{0}, R^{-1 / 2}\right) \cap S}\left|f_{\tau}\right|^{2} d \sigma(\xi) \leq \frac{c}{R^{(b+1) / 2}}
$$

for some absolute constant $c$. Using part (i) of Proposition 7.2 we notice that the supports of the $f_{\tau, i}$ are contained in neighborhoods $\tau^{\prime}$ of $\tau$, which, after choosing $C$ sufficiently large (in order for $R^{-1 / 2}$ to be sufficiently small), define a decomposition $\left\{\tau^{\prime}\right\}$ of $S$ of multiplicity $2 m$. Letting $g_{\tau, i}=c^{-1 / 2} f_{\tau, i}$ and $g_{i}=c^{-1 / 2} f_{i}$, we now see that $g_{i} \in \Lambda(R, K, 2 m, b)$.

By Lemma 8 -B and Lemma 8 -A (applied with $k=2 D$ ), we know that

$$
\sum_{i \in I} \int\left|f_{\tau, i}\right|^{2} d \sigma(\xi) \lesssim D \int\left|f_{\tau}\right|^{2} d \sigma(\xi)
$$

so that

$$
\sum_{i \in I} \sum_{\tau} \int\left|f_{\tau, i}\right|^{2} d \sigma(\xi) \lesssim D \sum_{\tau} \int\left|f_{\tau}\right|^{2} d \sigma(\xi)
$$

From among the $\sim D^{3}$ indices $i \in I$ that satisfy (29), we can therefore pick a particular index $i$ that also satisfies

$$
\sum_{\tau} \int\left|f_{\tau, i}\right|^{2} d \sigma(\xi) \lesssim D^{-2} \sum_{\tau} \int\left|f_{\tau}\right|^{2} d \sigma(\xi)
$$

For sufficiently large $D$, we therefore have

$$
\sum_{\tau} \int\left|g_{\tau, i}\right|^{2} d \sigma(\xi) \leq \frac{1}{2} \sum_{\tau} \int\left|f_{\tau}\right|^{2} d \sigma(\xi) .
$$

Lemma 9-A now tells us that

$$
\begin{aligned}
\int_{B_{R}} & \operatorname{Br}_{\beta} E f(x)^{p} H(x) d x \lesssim D^{3} \int_{B_{R} \cap O_{i}^{\prime}} \operatorname{Br}_{\beta} E f(x)^{p} H(x) d x \\
& \lesssim D^{3} \int_{B_{R}} \operatorname{Br}_{2 \beta} E g_{i}(x)^{p} H(x) d x+D^{3}\left(R^{-N+1} \sum_{\tau}\left\|f_{\tau}\right\|_{L^{1}(S)}\right)^{p} A_{\alpha}(H)^{q_{1}} R^{3} \\
& \lesssim D^{3} \int_{B_{R}} \operatorname{Br}_{2 \beta} E g_{i}(x)^{p} H(x) d x+R^{-N+7} A_{\alpha}(H)^{q_{1}}\left(\sum_{\tau}\left\|f_{\tau}\right\|_{L^{2}(S)}^{2}\right)^{(3 / 2)+\epsilon}
\end{aligned}
$$

where we have used the assumption $3<p \leq 4$ as well as (25). By induction on $\sum_{\tau} \int\left|f_{\tau}\right|^{2} d \sigma(\xi)$, we can apply (27) to $g_{i}$. We get

$$
\begin{aligned}
& \int_{B_{R}} \operatorname{Br}_{2 \beta} E g_{i}(x)^{p} H(x) d x \\
& \leq C_{\epsilon} R^{q_{0} \epsilon} A_{\alpha}(H)^{q_{1}}\left(\sum_{\tau} \int\left|g_{\tau, i}\right|^{2} d \sigma(\xi)\right)^{(3 / 2)+\epsilon} R^{\delta_{\text {trans }} \log \left(4 K^{\epsilon} \beta m\right)},
\end{aligned}
$$


so that

$$
\begin{aligned}
\int_{B_{R}} & \operatorname{Br}_{\beta} E f(x)^{p} H(x) d x \lesssim D^{3} \int_{B_{R} \cap O_{i}^{\prime}} \operatorname{Br}_{\beta} E f(x)^{p} H(x) d x \\
\leq & C^{\prime} D^{3} C_{\epsilon} R^{q_{0} \epsilon} A_{\alpha}(H)^{q_{1}}\left(D^{-2} \sum_{\tau} \int\left|f_{\tau}\right|^{2} d \sigma(\xi)\right)^{(3 / 2)+\epsilon} R^{\delta_{\text {trans }} \log \left(4 K^{\epsilon} \beta m\right)} \\
& +C^{\prime} R^{-N+7} A_{\alpha}(H)^{q_{1}}\left(\sum_{\tau}\left\|f_{\tau}\right\|_{L^{2}(S)}^{2}\right)^{(3 / 2)+\epsilon} \\
= & \left(C^{\prime} D^{-2 \epsilon} R^{(\log 4) \delta_{\text {trans }}}\right) C_{\epsilon} R^{q_{0} \epsilon} A_{\alpha}(H)^{q_{1}}\left(\sum_{\tau} \int\left|f_{\tau}\right|^{2} d \sigma(\xi)\right)^{(3 / 2)+\epsilon} \\
& \times R^{\delta_{\text {trans }} \log \left(K^{\epsilon} \beta m\right)}+C^{\prime} R^{-N+7} A_{\alpha}(H)^{q_{1}}\left(\sum_{\tau}\left\|f_{\tau}\right\|_{L^{2}(S)}^{2}\right)^{(3 / 2)+\epsilon}
\end{aligned}
$$

To close the induction, it just suffices to prove that

$$
C^{\prime} D^{-2 \epsilon} R^{(\log 4) \delta_{\text {trans }}}+C^{\prime} R^{-N+7} \leq 1 .
$$

Since

$$
D^{-2 \epsilon} R^{(\log 4) \delta_{\text {trans }}}=R^{-2 \epsilon \delta_{\operatorname{deg}}} R^{(\log 4) \delta_{\text {trans }}}=R^{-2 \epsilon^{3}+(\log 4) \epsilon^{6}},
$$

it follows that the exponent of $R$ in both terms is negative and the induction closes.

10.2. The algebraic case. Returning to the decomposition

$$
\begin{aligned}
& \int_{B_{R}} \operatorname{Br}_{\beta} E f(x)^{p} H(x) d x \\
& \quad=\sum_{i} \int_{B_{R} \cap O_{i}^{\prime}} \operatorname{Br}_{\beta} E f(x)^{p} H(x) d x+\int_{B_{R} \cap W} \operatorname{Br}_{\beta} E f(x)^{p} H(x) d x,
\end{aligned}
$$

we now assume that the contribution from the cell-wall $W$ dominates. By Lemma 9-B we know that

$$
\begin{aligned}
\int_{B_{R}} \operatorname{Br}_{\beta} E f(x)^{p} H(x) d x \lesssim & \sum_{j, I} \int_{B_{j} \cap W} \operatorname{Br}_{2 \beta} E f_{I, j, \operatorname{trans}}(x)^{p} H(x) d x \\
& +K^{100 p} \sum_{j} \int_{B_{j} \cap W} \operatorname{Bil}_{P, \delta} E f_{j, \operatorname{tang}}(x)^{p} H(x) d x \\
& +O\left(R^{-N+1} \sum_{\tau}\left\|f_{\tau}\right\|_{L^{1}(S)}\right)^{p} A_{\alpha}(H)^{q_{1}} R^{3}
\end{aligned}
$$

If the final $O$-term dominates, then the conclusion holds trivially (by using the assumption $3<p \leq 4$ and (25) as before).

If the tangential term dominates, then, recalling that $|\{j\}| \lesssim R^{3 \delta}$, we see that the conclusion holds by (26).

So we may assume that

$$
\int_{B_{R}} \operatorname{Br}_{\beta} E f(x)^{p} H(x) d x \lesssim \sum_{j, I} \int_{B_{j} \cap W} \operatorname{Br}_{2 \beta} E f_{I, j, \text { trans }}(x)^{p} H(x) d x .
$$

Each ball $B_{j}$ has radius $R^{1-\delta}$. By induction on the radius, we can therefore apply (27) to each integral on the right-hand side as soon as we verify that $f_{I, j \text {, trans }} \in$ $\Lambda\left(R^{1-\delta}, K, 2 m, b\right)$. (The multiplicity of the new decomposition of $S$ is $2 m$ for the 
same reason given above concerning the decomposition $\left\{\tau^{\prime}\right\}$ associated with the function $g_{i}$.) By Lemma 8 -A (applied to a single subset $\mathbb{T}_{j \text {,trans }} \subset \mathbb{T}$ ), we have

$$
\int_{B\left(\xi_{0}, R^{-(1-\delta) / 2}\right) \cap S}\left|f_{\tau, j, \text { trans }}\right|^{2} d \sigma(\xi) \lesssim \int_{B\left(\xi_{0}, R^{-(1-\delta) / 2}\right) \cap S}\left|f_{\tau}\right|^{2} d \sigma(\xi)
$$

for all $\xi_{0} \in S$. Using (24), we see that there is an absolute constant $c$ such that

$$
\int_{B\left(\xi_{0}, R^{-(1-\delta) / 2}\right) \cap S}\left|f_{\tau, j, \text { trans }}\right|^{2} d \sigma(\xi) \leq \frac{c R^{\delta}}{R^{(b+1) / 2}} \leq \frac{c R^{(b+1) \delta / 2}}{R^{(b+1) / 2}}=\frac{c}{R^{(1-\delta)(b+1) / 2}},
$$

where we have used the assumption that $b \geq 1$. Therefore,

$$
c^{-1 / 2} f_{I, j, \text { trans }} \in \Lambda\left(R^{1-\delta}, K, 2 m, b\right)
$$

and we may apply (27) to each of the integrals on the right-hand side of (30) to get

$$
\begin{aligned}
& \int_{B_{j}}\left(\operatorname{Br}_{2 \beta} E f_{I, j, \text { trans }}(x)^{p} H(x) d x\right. \\
& \leq c^{2} C_{\epsilon} R^{(1-\delta) q_{0} \epsilon} A_{\alpha}(H)^{q_{1}}\left(\sum_{\tau} \int\left|f_{\tau, j, \text { trans }}\right|^{2} d \sigma(\xi)\right)^{(3 / 2)+\epsilon} \\
& \quad \times R^{(1-\delta) \delta_{\text {trans }} \log \left(4 K^{\epsilon} \beta m\right)} .
\end{aligned}
$$

From Lemma 8-C] we know that a given tube in $\mathbb{T}$ lies in $\mathbb{T}_{j, \text { trans }}$ for at most Poly $(D)$ values of $j$, so Lemma $8-A$ (applied with $k=\operatorname{Poly}(D)$ ) implies that

$$
\sum_{j} \int\left|f_{\tau, j, \operatorname{trans}}\right|^{2} d \sigma(\xi) \lesssim \operatorname{Poly}(D) \int\left|f_{\tau}\right|^{2} d \sigma(\xi)
$$

and hence

$$
\left(\sum_{j} \sum_{\tau \in I} \int\left|f_{\tau, j, \operatorname{trans}}\right|^{2} d \sigma(\xi)\right)^{\frac{3}{2}+\epsilon} \lesssim \operatorname{Poly}(D)\left(\sum_{\tau} \int\left|f_{\tau}\right|^{2} d \sigma(\xi)\right)^{\frac{3}{2}+\epsilon}
$$

and hence

$$
\sum_{j}\left(\sum_{\tau \in I} \int\left|f_{\tau, j, \operatorname{trans}}\right|^{2} d \sigma(\xi)\right)^{\frac{3}{2}+\epsilon} \lesssim \operatorname{Poly}(D)\left(\sum_{\tau} \int\left|f_{\tau}\right|^{2} d \sigma(\xi)\right)^{\frac{3}{2}+\epsilon}
$$

with the implicit constant independent of the cardinality of $\{j\}$, and hence

$$
\sum_{j, I}\left(\sum_{\tau \in I} \int\left|f_{\tau, j, \text { trans }}\right|^{2} d \sigma(\xi)\right)^{\frac{3}{2}+\epsilon} \lesssim \operatorname{Poly}(D)\left(\sum_{\tau} \int\left|f_{\tau}\right|^{2} d \sigma(\xi)\right)^{\frac{3}{2}+\epsilon}
$$

with the implicit constant depending on the cardinality of $\{I\}$ (which only depends on $K$, which is acceptable). Thus

$$
\begin{aligned}
\int_{B_{R}} & \operatorname{Br}_{\beta} E f(x)^{p} H(x) d x \\
\leq & C^{\prime} \operatorname{Poly}(D) C_{\epsilon} R^{(1-\delta) q_{0} \epsilon} A_{\alpha}(H)^{q_{1}}\left(\sum_{\tau} \int\left|f_{\tau}\right|^{2} d \sigma(\xi)\right)^{\frac{3}{2}+\epsilon} \\
& \times R^{\delta_{\text {trans }} \log \left(4 K^{\epsilon} \beta m\right)} \\
= & \left(C^{\prime} \operatorname{Poly}(D) R^{-\delta q_{0} \epsilon} R^{(\log 4) \delta_{\text {trans }}}\right) C_{\epsilon} R^{q_{0} \epsilon} A_{\alpha}(H)^{q_{1}} \\
& \times\left(\sum_{\tau} \int\left|f_{\tau}\right|^{2} d \sigma(\xi)\right)^{\frac{3}{2}+\epsilon} R^{\delta_{\text {trans }} \log \left(K^{\epsilon} \beta m\right)}
\end{aligned}
$$


To close the induction, we just have to prove that

$$
C^{\prime} \operatorname{Poly}(D) R^{-\delta q_{0} \epsilon} R^{(\log 4) \delta_{\text {trans }}} \leq 1 .
$$

But

$$
C^{\prime} \operatorname{Poly}(D) R^{-\delta q_{0} \epsilon} R^{(\log 4) \delta_{\text {trans }}} \leq R^{C^{\prime \prime} \delta_{\operatorname{deg}}-\delta q_{0} \epsilon+(\log 4) \delta_{\text {trans }}} \leq R^{C^{\prime \prime} \epsilon^{4}-(1 / 2) \epsilon^{3}+(\log 4) \epsilon^{6}},
$$

where we have used the assumption that $q_{0} \geq 1 / 2$, so the induction closes provided $\epsilon$ is sufficiently small.

\section{Estimates ON THE BROAD PART}

This section and the next form the bulk of the proof of Theorem 1.1. In this section, we use Theorem 9.1 to estimate various $L^{p}$ norms of the broad part of $E f$ on the ball $B_{R}$ with respect to the measure $H(x) d x$, where $H$ is a weight of dimension $\alpha$, as defined in the Introduction. In view of the conditional formulation of Theorem 9.1. this will be achieved by estimating the tangential part of $E f$.

Following [8], we cover $B_{j} \cap W$ with cubes $Q$ of side length $R^{1 / 2}$. For each cube $Q$, we let $\mathbb{T}_{j, \text { tang, } Q}$ be the set of tubes in $\mathbb{T}_{j, \text { tang }}$ that intersect $Q$. We know that

$$
E f_{\tau, j, \text { tang }}=\sum_{T \in \mathbb{T}_{j, \text { tang }}} E f_{\tau, T}=\sum_{T \in \mathbb{T}_{j, \text { tang }, Q}} E f_{\tau, T}+\sum_{T \in \mathbb{T}_{j, \operatorname{tang}} \backslash T_{j, \text { tang }, Q}} E f_{\tau, T} .
$$

Let $x \in Q$. If $x \in T$, then $T$ must intersect $Q$, and it follows that $T \in \mathbb{T}_{j, \operatorname{tang}, Q}$. If $x \notin T$ and $T \in \mathbb{T}(\theta)$, then $\left|E f_{\tau, T}(x)\right| \lesssim R^{-N}\left\|f_{\tau}\right\|_{L^{1}(\theta)}$, so

$$
\begin{aligned}
\sum_{T \in \mathbb{T}_{j, \text { tang } \backslash T_{j, \text { tang }, Q}}\left|E f_{\tau, T}(x)\right|} & =\sum_{\theta} \sum_{T \in\left(\mathbb{T}(\theta) \cap \mathbb{T}_{j, \text { tang }} \backslash T_{j, \text { tang }, Q}\right.}\left|E f_{\tau, T}(x)\right| \\
& \lesssim \sum_{\theta} R^{-N}\left\|f_{\tau}\right\|_{L^{1}(\theta)} \\
& =R^{-N}\left\|f_{\tau}\right\|_{L^{1}(S)},
\end{aligned}
$$

and so

$$
E f_{\tau, j, \operatorname{tang}}(x)=\sum_{T \in \mathbb{T}_{j, \text { tang }, Q}} E f_{\tau, T}(x)+O\left(R^{-N}\left\|f_{\tau}\right\|_{L^{1}(S)}\right) .
$$

Because of the definition of $T_{j, \text { tang }}$, it turns out that all the tubes in $T_{j, \operatorname{tang}, Q}$ are nearly coplanar (provided $\delta$ is sufficiently small for the radius of the tubes to be smaller than the radius of $B_{j}$, i.e. provided $R^{(1 / 2)+\delta} \leq R^{1-\delta}$ ). This led Guth to use the Córdoba $L^{4}$ argument and obtain the following bilinear estimate on $Q$.

Lemma 11-A (Lemma 3.10 in [8]). Suppose $0<\delta \leq 1 / 4$. It $\tau_{1}$ and $\tau_{2}$ are nonadjacent caps, then

$$
\begin{aligned}
& \int_{Q}\left|E f_{\tau_{1}, j, \text { tang }}\right|^{2}\left|E f_{\tau_{2}, j, \text { tang }}\right|^{2} d x \\
& \lesssim R^{O(\delta)} R^{-1 / 2}\left(\sum_{T_{1} \in \mathbb{T}_{j, \text { tang }, Q}}\left\|f_{\tau_{1}, T_{1}}\right\|_{L^{2}(S)}^{2}\right)\left(\sum_{T_{2} \in \mathbb{T}_{j, \text { tang }, Q}}\left\|f_{\tau_{2}, T_{2}}\right\|_{L^{2}(S)}^{2}\right) \\
& \quad+O\left(R^{-N+2}\left(\sum_{\tau}\left\|f_{\tau}\right\|_{L^{2}(S)}^{2}\right)^{2}\right) .
\end{aligned}
$$


To upgrade the estimate in Lemma 11-A from an estimate on $Q$ to an estimate on $B_{j} \cap W$, Guth then considered the square function

$$
S_{\tau, j, \text { tang }}=\left(\sum_{T \in \mathbb{T}_{j, \text { tang }}}\left(\chi_{7 T} R^{-1 / 2}\left\|f_{\tau, T}\right\|_{L^{2}(S)}\right)^{2}\right)^{1 / 2},
$$

where $7 T$ is the tube with the same core line as $T$ but seven times the radius. If $T \cap Q \neq \emptyset$, then $Q \subset 7 T$. So

$$
S_{\tau, j, \text { tang }}^{2} \geq \sum_{T \in \mathbb{T}_{j, \text { tang }, Q}}\left(\chi_{Q} R^{-1 / 2}\left\|f_{\tau, T}\right\|_{L^{2}(S)}\right)^{2}=\frac{\chi_{Q}}{R} \sum_{T \in \mathbb{T}_{j, \text { tang }, Q}}\left\|f_{\tau, T}\right\|_{L^{2}(S)}^{2},
$$

and so

$$
S_{\tau_{1}, j, \text { tang }}^{2} S_{\tau_{2}, j, \text { tang }}^{2} \geq \frac{\chi_{Q}}{R^{2}}\left(\sum_{T_{1} \in \mathbb{T}_{j, \text { tang }, Q}}\left\|f_{\tau_{1}, T_{1}}\right\|_{L^{2}(S)}^{2}\right)\left(\sum_{T_{2} \in \mathbb{T}_{j, \text { tang }, Q}}\left\|f_{\tau_{2}, T_{2}}\right\|_{L^{2}(S)}^{2}\right),
$$

which gives

$$
\begin{aligned}
\int_{Q} S_{\tau_{1}, j, \text { tang }}^{2} S_{\tau_{2}, j, \text { tang }}^{2} d x \\
\quad \geq \frac{|Q|}{R^{2}}\left(\sum_{T_{1} \in \mathbb{T}_{j, \text { tang }, Q}}\left\|f_{\tau_{1}, T_{1}}\right\|_{L^{2}(S)}^{2}\right)\left(\sum_{T_{2} \in \mathbb{T}_{j, \text { tang }, Q}}\left\|f_{\tau_{2}, T_{2}}\right\|_{L^{2}(S)}^{2}\right) \\
=R^{-1 / 2}\left(\sum_{T_{1} \in \mathbb{T}_{j, \text { tang }, Q}}\left\|f_{\tau_{1}, T_{1}}\right\|_{L^{2}(S)}^{2}\right)\left(\sum_{T_{2} \in \mathbb{T}_{j, \text { tang }, Q}}\left\|f_{\tau_{2}, T_{2}}\right\|_{L^{2}(S)}^{2}\right) .
\end{aligned}
$$

Lemma 11-A now implies that

$$
\begin{aligned}
& \int_{Q}\left|E f_{\tau_{1}, j, \text { tang }}\right|^{2}\left|E f_{\tau_{2}, j, \text { tang }}\right|^{2} d x \\
& \quad \lesssim R^{O(\delta)} \int_{Q} S_{\tau_{1}, j, \text { tang }}^{2} S_{\tau_{2}, j, \text { tang }}^{2} d x+O\left(R^{-N+2}\left(\sum_{\tau}\left\|f_{\tau}\right\|_{L^{2}(S)}^{2}\right)^{2}\right) .
\end{aligned}
$$

Summing over all the $Q$ covering $B_{j} \cap W$, and expanding the definition of the square function, this becomes

$$
\begin{aligned}
& \int_{B_{j} \cap W}\left|E f_{\tau_{1}, j, \text { tang }}\right|^{2}\left|E f_{\tau_{2}, j, \text { tang }}\right|^{2} d x \\
& \lesssim R^{O(\delta)} \int_{B_{j} \cap W} S_{\tau_{1}, j, \text { tang }}^{2} S_{\tau_{2}, j, \text { tang }}^{2} d x+O\left(R^{-N+4}\left(\sum_{\tau}\left\|f_{\tau}\right\|_{L^{2}(S)}^{2}\right)^{2}\right) \\
& \lesssim R^{O(\delta)} \sum_{T_{1}, T_{2} \in \mathbb{T}_{j, \text { tang }}} R^{-2}\left\|f_{\tau_{1}, T_{1}}\right\|_{L^{2}(S)}^{2}\left\|f_{\tau_{2}, T_{2}}\right\|_{L^{2}(S)}^{2} \int_{B_{j} \cap W} \chi_{7 T_{1}} \chi_{7 T_{2}} d x \\
& \quad+O\left(R^{-N+4}\left(\sum_{\tau}\left\|f_{\tau}\right\|_{L^{2}(S)}^{2}\right)^{2}\right) .
\end{aligned}
$$

Since $T_{1}$ comes from the wave packet decomposition of $f_{\tau_{1}}$ and $T_{2}$ comes from the wave packet decomposition of $f_{\tau_{2}}$, the angle between $v\left(T_{1}\right)$ and $v\left(T_{2}\right)$ is $\gtrsim K^{-1}$. So

$$
\int_{\mathbb{R}^{3}} \chi_{7 T_{1}} \chi_{7 T_{2}} d x \lesssim \frac{R^{(1 / 2)+\delta}}{K^{-1}} R^{(1 / 2)+\delta} R^{(1 / 2)+\delta}=K R^{(3 / 2)+3 \delta}
$$


Inserting this bound in the last inequality, Guth obtained

$$
\begin{aligned}
& \int_{B_{j} \cap W}\left|E f_{\tau_{1}, j, \text { tang }}\right|^{2}\left|E f_{\tau_{2}, j, \text { tang }}\right|^{2} d x \\
& \therefore R^{O(\delta)} R^{-1 / 2}\left(\sum_{T_{1} \in \mathbb{T}_{j, \text { tang }}}\left\|f_{\tau_{1}, T_{1}}\right\|_{L^{2}(S)}^{2}\right)\left(\sum_{T_{2} \in \mathbb{T}_{j, \text { tang }}}\left\|f_{\tau_{2}, T_{2}}\right\|_{L^{2}(S)}^{2}\right) \\
& \quad+O\left(R^{-N+4}\left(\sum_{\tau}\left\|f_{\tau}\right\|_{L^{2}(S)}^{2}\right)^{2}\right) .
\end{aligned}
$$

We are now in position to state our first estimate on the broad part of $E f$.

Theorem 11.1. Suppose $3 / 2<\alpha \leq 3, H$ is a weight of dimension $\alpha, b \geq 1$, and $p=2(4 \alpha+3 b) /(2 \alpha+2 b+1)$.

Then there is a constant $c_{0}$, which is independent of $b$, such that to every $0<\epsilon \leq$ $\min \left[c_{0},(p-3) / 2\right]$ there are constants $K=K(\epsilon)$ and $C_{\epsilon}$ so that $\lim _{\epsilon \rightarrow 0} K(\epsilon)=\infty$ and

$$
\int_{B_{R}} \operatorname{Br}_{K^{-\epsilon}} E f(x)^{p} H(x) d x \leq C_{\epsilon} R^{(b+1) \epsilon / 2} A_{\alpha}(H)^{1-(p / 4)}\|f\|_{L^{2}(S)}^{3+2 \epsilon}
$$

whenever $R \geq 1, f \in L^{2}(S)$, and

$$
\int_{B\left(\xi_{0}, R^{-1 / 2}\right) \cap S}|f(\xi)|^{2} d \sigma(\xi) \leq \frac{1}{R^{(b+1) / 2}}
$$

for all $\xi_{0} \in S$.

Proof. In view of Theorem 9.1. we have to establish (26). To guarantee the independence of $c_{0}$ from $b$, we will again be careful to check that all constants appearing in this proof are independent of this parameter.

Starting with (31) and noticing that $3<p<4$, we see by Hölder's inequality that

$$
\begin{aligned}
& \int_{B_{j} \cap W} \operatorname{Bil}_{P, \delta} E f_{j, \text { tang }}(x)^{p} H(x) d x \\
& \underset{\tau_{1}, \tau_{2}}{\lesssim} \sum_{\text {non-adjacent }} \int_{B_{j} \cap W}\left|E f_{\tau_{1}, j, \text { tang }}\right|^{p / 2}\left|E f_{\tau_{2}, j, \text { tang }}\right|^{p / 2} H(x) d x \\
& \leq \sum_{\tau_{1}, \tau_{2} \text { non-adjacent }}\left(\int_{B_{j} \cap W}\left|E f_{\tau_{1}, j, \text { tang }}\right|^{2}\left|E f_{\tau_{2}, j, \text { tang }}\right|^{2} d x\right)^{\frac{p}{4}}\left(A_{\alpha}(H) R^{\alpha}\right)^{1-\frac{p}{4}} \\
& \lesssim \quad R^{O(\delta)} A_{\alpha}(H)^{1-\frac{p}{4}} R^{-\frac{p}{8}+\alpha-\alpha \frac{p}{4}} \sum_{\tau_{1}, \tau_{2}} J^{\frac{p}{4}} \\
& +O\left(\left(A_{\alpha}(H) R^{\alpha}\right)^{1-\frac{p}{4}} R^{(-N+4)(p / 4)}\left(\sum_{\tau}\left\|f_{\tau}\right\|_{L^{2}(S)}^{2}\right)^{\frac{p}{2}}\right),
\end{aligned}
$$

where

$$
J=\left(\sum_{T_{1} \in \mathbb{T}_{j, \text { tang }}}\left\|f_{\tau_{1}, T_{1}}\right\|_{L^{2}(S)}^{2}\right)\left(\sum_{T_{2} \in \mathbb{T}_{j, \text { tang }}}\left\|f_{\tau_{2}, T_{2}}\right\|_{L^{2}(S)}^{2}\right) .
$$

Following $[8$, we will bound $J$ in two different ways. 
By part (i) of Proposition 7.2, we have

$$
\begin{aligned}
& \sum_{T_{1} \in \mathbb{T}_{j, \text { tang }}}\left\|f_{\tau_{1}, T_{1}}\right\|_{L^{2}(S)}^{2} \\
& \quad=\sum_{\theta} \sum_{T_{1} \in \mathbb{T}(\theta) \cap \mathbb{T}_{j, \text { tang }}}\left\|f_{\tau_{1}, T_{1}}\right\|_{L^{2}(S)}^{2} \lesssim \sum_{\theta}\left\|f_{\tau_{1}}\right\|_{L^{2}(\theta)}^{2} \lesssim\left\|f_{\tau_{1}}\right\|_{L^{2}(S)}^{2} .
\end{aligned}
$$

Likewise, $\sum_{T_{2} \in \mathbb{T}_{j, \text { tang }}}\left\|f_{\tau_{2}, T_{2}}\right\|_{L^{2}(S)}^{2} \lesssim\left\|f_{\tau_{2}}\right\|_{L^{2}(S)}^{2}$. This gives the bound

$$
J \lesssim\left\|f_{\tau_{1}}\right\|_{L^{2}(S)}^{2}\left\|f_{\tau_{2}}\right\|_{L^{2}(S)}^{2}
$$

On the other hand, Lemma 8 -D tells us that $\mathbb{T}_{j, \text { tang }}$ contains tubes in only $R^{O(\delta)} R^{1 / 2}$ different directions, so

$$
\begin{aligned}
\sum_{T_{1} \in \mathbb{T}_{j, \operatorname{tang}}}\left\|f_{\tau_{1}, T_{1}}\right\|_{L^{2}(S)}^{2} & \lesssim \sum_{R^{(1 / 2)+O(\delta)} \operatorname{caps} \theta} \sum_{T_{1} \in \mathbb{T}(\theta)}\left\|f_{\tau_{1}, T_{1}}\right\|_{L^{2}(S)}^{2} \\
& \lesssim \sum_{R^{(1 / 2)+O(\delta) \operatorname{caps} \theta}} \int_{\theta}\left|f_{\tau_{1}}\right|^{2} d \sigma(\xi) \\
& \lesssim \frac{R^{(1 / 2)+O(\delta)}}{R^{(b+1) / 2}}=R^{O(\delta)} R^{-b / 2}
\end{aligned}
$$

where on the second line we used part (i) of Proposition 7.2 and on the third line the fact that $\int_{\theta}\left|f_{\tau}\right|^{2} d \sigma(\xi) \lesssim R^{-(b+1) / 2}$. Likewise with $\tau_{1}, T_{1}$ replaced by $\tau_{2}, T_{2}$. Thus

$$
J \lesssim R^{O(\delta)} R^{-b}
$$

Putting the two bounds we now have on $J$ together, we see that

$$
\begin{aligned}
\sum_{\tau_{1}, \tau_{2}} J^{\frac{p}{4}} & \lesssim\left(\sum_{\tau_{1}, \tau_{2}} J\right)^{\frac{p}{4}}=\left(\sum_{\tau_{1}, \tau_{2}} J\right)^{(p-3-2 \epsilon) / 4}\left(\sum_{\tau_{1}, \tau_{2}} J\right)^{(3+2 \epsilon) / 4} \\
& \lesssim R^{O(\delta)} R^{-b(p-3-2 \epsilon) / 4}\left(\sum_{\tau_{1}, \tau_{2}}\left\|f_{\tau_{1}}\right\|_{L^{2}(S)}^{2}\left\|f_{\tau_{2}}\right\|_{L^{2}(S)}^{2}\right)^{(3+2 \epsilon) / 4} \\
& =R^{O(\delta)} R^{-b(p-3-2 \epsilon) / 4}\left(\sum_{\tau}\left\|f_{\tau}\right\|_{L^{2}(S)}^{2}\right)^{(3 / 2)+\epsilon}
\end{aligned}
$$

provided $p-3-2 \epsilon \geq 0$. We note that since $p-3-2 \epsilon<1-2 \epsilon$, all the implicit constants remain independent of $b$. Therefore,

$$
\begin{aligned}
\int_{B_{j} \cap W} & \operatorname{Bil}_{P, \delta} E f_{j, \operatorname{tang}}(x)^{p} H(x) d x \\
\lesssim & R^{O(\delta)} A_{\alpha}(H)^{1-\frac{p}{4}} R^{-\frac{p}{8}+\alpha-\alpha \frac{p}{4}} R^{-b \frac{p-3-2 \epsilon}{4}}\left(\sum_{\tau}\left\|f_{\tau}\right\|_{L^{2}(S)}^{2}\right)^{\frac{3}{2}+\epsilon} \\
& +O\left(\left(A_{\alpha}(H) R^{\alpha}\right)^{1-\frac{p}{4}} R^{(-N+4)(p / 4)}\left(\sum_{\tau}\left\|f_{\tau}\right\|_{L^{2}(S)}^{2}\right)^{\frac{p}{2}}\right) .
\end{aligned}
$$


Recalling from (25) that $\int\left|f_{\tau}\right|^{2} d \sigma \lesssim R^{-(b-1) / 2} \leq 1$, we have

$$
\begin{aligned}
\left(\sum_{\tau}\left\|f_{\tau}\right\|_{L^{2}(S)}^{2}\right)^{\frac{p}{2}} & =\left(\sum_{\tau}\left\|f_{\tau}\right\|_{L^{2}(S)}^{2}\right)^{\frac{p-3-2 \epsilon}{2}}\left(\sum_{\tau}\left\|f_{\tau}\right\|_{L^{2}(S)}^{2}\right)^{\frac{3}{2}+\epsilon} \\
& \lesssim\left(\sum_{\tau}\left\|f_{\tau}\right\|_{L^{2}(S)}^{2}\right)^{\frac{3}{2}+\epsilon}
\end{aligned}
$$

provided $p-3-2 \epsilon \geq 0$. Thus

$$
\begin{aligned}
\int_{B_{j} \cap W} & \operatorname{Bil}_{P, \delta} E f_{j, \operatorname{tang}}(x)^{p} H(x) d x \\
& \lesssim R^{O(\delta)} A_{\alpha}(H)^{1-\frac{p}{4}} R^{\frac{b \epsilon}{2}} R^{\alpha+\frac{3 b}{4}} R^{-\frac{p}{8}(1+2 \alpha+2 b)}\left(\sum_{\tau}\left\|f_{\tau}\right\|_{L^{2}(S)}^{2}\right)^{\frac{3}{2}+\epsilon} \\
\quad= & R^{O(\delta)} R^{b \epsilon / 2} A_{\alpha}(H)^{1-(p / 4)}\left(\sum_{\tau}\left\|f_{\tau}\right\|_{L^{2}(S)}^{2}\right)^{(3 / 2)+\epsilon} .
\end{aligned}
$$

We note that the implicit constant in the $R^{O(\delta)}$ factor does not depend on $b$.

Invoking Theorem 9.1 (with $q_{2}=b / 2, q_{1}=1-(p / 4)$, and $\left.q_{0}=(1 / 4)+(b / 2)\right)$, we conclude that to every sufficiently small $\epsilon$ there are constants $K=K(\epsilon)$ and $C_{\epsilon}$ such that $\lim _{\epsilon \rightarrow 0} K(\epsilon)=\infty$ and

$$
\begin{aligned}
& \int_{B_{R}} \operatorname{Br}_{\beta} E f(x)^{p} H(x) d x \\
& \quad \leq C_{\epsilon} R^{\epsilon / 4} R^{b \epsilon / 2} A_{\alpha}(H)^{1-(p / 4)}\left(\sum_{\tau}\left\|f_{\tau}\right\|_{L^{2}(S)}^{2}\right)^{(3 / 2)+\epsilon} R^{\delta \text { trans } \log \left(K^{\epsilon} \beta m\right)}
\end{aligned}
$$

for all $\beta \geq K^{-\epsilon}, m \geq 1, R \geq 1$, and $f \in \Lambda(R, K, m, b)$.

Now suppose $f \in L^{2}(S)$ satisfies

$$
\int_{B\left(\xi_{0}, R^{-1 / 2}\right) \cap S}|f(\xi)|^{2} d \sigma(\xi) \leq \frac{1}{R^{(b+1) / 2}}
$$

for all $\xi_{0} \in S$. Writing $f=\sum_{\tau} f_{\tau}$ with $\operatorname{supp} f_{\tau} \subset \tau$ and $\left(\operatorname{supp} f_{\tau}\right) \cap\left(\operatorname{supp} f_{\tau^{\prime}}\right)=\emptyset$ if $\tau \neq \tau^{\prime}$, we see that $f \in \Lambda(R, K, m, b)$ and $\sum_{\tau}\left\|f_{\tau}\right\|_{L^{2}(S)}^{2}=\|f\|_{L^{2}(S)}^{2}$. Applying the above estimate with $\beta=K^{-\epsilon}$, we obtain the desired result.

Remark 11.1. In dimension $n=2$, one needs to bound the broad part of $E f$ by $\left(\sum_{\tau}\left\|f_{\tau}\right\|_{L^{2}(S)}^{2}\right)^{2+\epsilon}$ rather than $\left(\sum_{\tau}\left\|f_{\tau}\right\|_{L^{2}(S)}^{2}\right)^{(3 / 2)+\epsilon}$ for the induction argument in the proof of Theorem 9.1 to work. In view of the argument leading to (32), however, one sees that replacing $(3 / 2)+\epsilon$ by $2+\epsilon$ requires $p / 4>1$. But when $p / 4>1$, one will not have the right exponent to apply Hölder's inequality in the second paragraph of the proof of Theorem 11.1, and as a result will not be able to exploit the dimensionality of $H$ and arrive at the desired estimate.

During the proof of the next theorem, we will need an estimate on

$$
\int_{B_{j}}\left|E f_{\tau, j, \operatorname{tang}}(x)\right|^{2} H(x) d x .
$$


BASSAM SHAYYA

In $[8$, where the function $H$ was not present, this was obtained via the standard local restriction estimate

$$
\int_{B(0, R)}|E f(x)|^{2} d x \lesssim R\|f\|_{L^{2}(S)}^{2}
$$

which holds for all $f \in L^{2}(S)$. One could still use this estimate here, because $\|H\|_{L^{\infty}} \leq 1$, but then one would be repeating word for word the argument from 8 and would end up with the same estimate on the broad part of $E f$ as in that paper. In order to improve matters - via the induction argument of the next section - we have to involve the factor $A_{\alpha}(H)$ in our estimate, and we, therefore, have to update the above local restriction estimate accordingly.

Lemma 11.1. Suppose $0<\alpha \leq 3$ and $H$ is a weight of dimension $\alpha$. Then

$$
\int_{B(0, R)}|E f(x)|^{2} H(x) d x \lesssim A_{\alpha}(H) R\|f\|_{L^{2}(S)}^{2}
$$

for all $R \geq 1$ and $f \in L^{2}(S)$.

Proof. We let $\eta$ be a $C_{0}^{\infty}$ function on $\mathbb{R}^{3}$ such that $|\widehat{\eta}| \geq 1$ on $B(0,1)$. Then

$$
\int_{B(0, R)}|E f(x)|^{2} H(x) d x \leq \int\left|E f(x) \widehat{\eta_{R^{-1}}}(x)\right|^{2} H(x) d x=\int|\widehat{F}(x)|^{2} H(x) d x,
$$

where $F=\eta_{R^{-1}} * f d \sigma$. Since $\eta$ is compactly supported and $R \geq 1, F * F$ is supported in a ball $B(0, C)$. We let $\phi$ be a Schwartz function on $\mathbb{R}^{3}$ such that $\phi=1$ on $B(0, C)$. Then $F * F=\phi(F * F)$, and, accordingly,

$$
\begin{aligned}
\int|\widehat{F}(x)|^{2} H(x) d x & =\int\left|\int \widehat{\phi}(x-y) \widehat{F * F}(y) d y\right| H(x) d x \\
& \leq \int|\widehat{F * F}(y)| \int|\widehat{\phi}(x-y)| H(x) d x d y .
\end{aligned}
$$

To estimate the inner integral, we let $B_{l}=B\left(y, 2^{l}\right)$, and observe that

$$
\begin{aligned}
\int|\widehat{\phi}(x-y)| H(x) d x & =\int_{B_{0}}|\widehat{\phi}(x-y)| H(x) d x+\sum_{l=1}^{\infty} \int_{B_{l} \backslash B_{l-1}}|\widehat{\phi}(x-y)| H(x) d x \\
& \leq \int_{B_{0}} \frac{C_{N} H(x) d x}{(1+|x-y|)^{N}}+\sum_{l=1}^{\infty} \int_{B_{l} \backslash B_{l-1}} \frac{C_{N} H(x) d x}{(1+|x-y|)^{N}} \\
& \lesssim A_{\alpha}(H) .
\end{aligned}
$$

Therefore,

$$
\int_{B(0, R)}|E f(x)|^{2} H(x) d x \lesssim A_{\alpha}(H) \int|\widehat{F * F}(y)| d y=A_{\alpha}(H) \int|F(\xi)|^{2} d \xi .
$$

By the definition of $F$, and the Cauchy-Schwarz inequality, we have

$$
|F(\xi)|^{2} \leq\left(\int\left|\eta_{R^{-1}}(\xi-\zeta)\right| d \sigma(\zeta)\right)\left(\int\left|\eta_{R^{-1}}(\xi-\zeta)\right||f(\zeta)|^{2} d \sigma(\zeta)\right) .
$$

Clearly,

$$
\int\left|\eta_{R^{-1}}(\xi-\zeta)\right| d \sigma(\zeta)=R^{3} \int|\eta(R(\xi-\zeta))| d \sigma(\zeta) \lesssim R^{3} \sigma\left(B\left(\xi, R^{-1}\right)\right) \lesssim R
$$


SO

$$
\int|F(\xi)|^{2} d \xi \lesssim R \int|f(\zeta)|^{2} \int\left|\eta_{R^{-1}}(\xi-\zeta)\right| d \xi d \sigma(\zeta)=R\|\eta\|_{L^{1}}\|f\|_{L^{2}(S)}^{2}
$$

and so

$$
\int_{B(0, R)}|E f(x)|^{2} H(x) d x \lesssim A_{\alpha}(H) R\|f\|_{L^{2}(S)}^{2}
$$

as claimed.

This brings us to our second estimate on the broad part of $E f$.

Theorem 11.2. Suppose $0<\alpha \leq 3, H$ is a weight of dimension $\alpha$, and $p=13 / 4$.

Then there is a constant $c$, with $0<c \leq(p-3) / 2$, such that to every $0<\epsilon<c$ there are constants $K=K(\epsilon)$ and $C_{\epsilon}$ so that $\lim _{\epsilon \rightarrow 0} K(\epsilon)=\infty$ and

$$
\int_{B_{R}} \operatorname{Br}_{K^{-\epsilon}} E f(x)^{p} H(x) d x \leq C_{\epsilon} R^{\epsilon} A_{\alpha}(H)^{2-(p / 2)}\|f\|_{L^{2}(S)}^{3+2 \epsilon}
$$

whenever $R \geq 1, f \in L^{2}(S)$, and

$$
\int_{B\left(\xi_{0}, R^{-1 / 2}\right) \cap S}|f(\xi)|^{2} d \sigma(\xi) \leq \frac{1}{R}
$$

for all $\xi_{0} \in S$.

Proof. The argument we use here is very close to the one presented in $\S 3.4$ of $[8]$. Our starting point is again (31), which gives the following $L^{4}$ bound on the bilinear term:

$$
\begin{aligned}
& \int_{B_{j} \cap W} \operatorname{Bil}_{P, \delta} E f_{j, \text { tang }}(x)^{4} H(x) d x \leq \int_{B_{j} \cap W} \operatorname{Bil}_{P, \delta} E f_{j, \text { tang }}(x)^{4} d x \\
& \lesssim \sum_{\tau_{1}, \tau_{2} \text { non-adjacent }} \int_{B_{j} \cap W}\left|E f_{\tau_{1}, j, \text { tang }}\right|^{2}\left|E f_{\tau_{2}, j, \text { tang }}\right|^{2} d x \lesssim R^{O(\delta)} R^{-1 / 2} M+\mathcal{E}
\end{aligned}
$$

where

$$
M=\sum_{\tau_{1}, \tau_{2}}\left(\sum_{T_{1} \in \mathbb{T}_{j, \text { tang }}}\left\|f_{\tau_{1}, T_{1}}\right\|_{L^{2}(S)}^{2}\right)\left(\sum_{T_{2} \in \mathbb{T}_{j, \text { tang }}}\left\|f_{\tau_{2}, T_{2}}\right\|_{L^{2}(S)}^{2}\right)
$$

and $\mathcal{E}=O\left(R^{-N+4}\left(\sum_{\tau}\left\|f_{\tau}\right\|_{L^{2}(S)}^{2}\right)^{2}\right)$.

On the other hand, Lemma 11.1 tells us that

$$
\begin{aligned}
\int_{B_{j} \cap W} & \operatorname{Bil}_{P, \delta} E f_{j, \text { tang }}(x)^{2} H(x) d x \\
& \lesssim \sum_{\tau_{1}, \tau_{2} \text { non-adjacent }} \int_{B_{j} \cap W}\left|E f_{\tau_{1}, j, \text { tang }} \| E f_{\tau_{2}, j, \text { tang }}\right| H(x) d x \\
& \leq \sum_{\tau_{1}, \tau_{2}}\left\|E f_{\tau_{1}, j, \text { tang }}\right\|\left\|_{L^{2}\left(B_{j}, H(x) d x\right)}\right\| E f_{\tau_{2}, j, \text { tang }} \|_{L^{2}\left(B_{j}, H(x) d x\right)} \\
& \lesssim A_{\alpha}(H) R^{1-\delta} \sum_{\tau_{1}, \tau_{2}}\left\|f_{\tau_{1}, j, \text { tang }}\right\|_{L^{2}(S)}\left\|f_{\tau_{2}, j, \text { tang }}\right\|_{L^{2}(S)} \\
& =A_{\alpha}(H) R^{1-\delta}\left(\sum_{\tau}\left\|f_{\tau, j, \text { tang }}\right\|_{L^{2}(S)}\right)^{2} \lesssim A_{\alpha}(H) R \sum_{\tau}\left\|f_{\tau, j, \text { tang }}\right\|_{L^{2}(S)}^{2} .
\end{aligned}
$$


Interpolating between the $L^{2}$ estimate and the $L^{4}$ estimate, we get for all $2 \leq$ $p \leq 4$,

$$
\begin{aligned}
\int_{B_{j} \cap W} & \operatorname{Bil}_{P, \delta} E f_{j, \text { tang }}(x)^{p} H(x) d x \\
= & \int_{B_{j} \cap W} \operatorname{Bil}_{P, \delta} E f_{j, \text { tang }}(x)^{(2-p / 2)(2)} \operatorname{Bil}_{P, \delta} E f_{j, \text { tang }}(x)^{(-1+p / 2)(4)} H(x) d x \\
\leq & \left(\int_{B_{j} \cap W} \operatorname{Bil}_{P, \delta} E f_{j, \text { tang }}(x)^{2} H(x) d x\right)^{2-\frac{p}{2}} \\
& \times\left(\int_{B_{j} \cap W} \operatorname{Bil}_{P, \delta} E f_{j, \text { tang }}(x)^{4} H(x) d x\right)^{-1+\frac{p}{2}} \\
\lesssim & \left(A_{\alpha}(H) R \sum_{\tau}\left\|f_{\tau, j, \operatorname{tang}}\right\|_{L^{2}(S)}^{2}\right)^{2-\frac{p}{2}}\left(R^{O(\delta)} R^{-1 / 2} M+\mathcal{E}\right)^{\frac{p}{2}-1} \\
\leq & R^{O(\delta)} A_{\alpha}(H)^{2-\frac{p}{2}} R^{\frac{5}{2}-\frac{3}{4} p}\left(\sum_{\tau}\left\|f_{\tau, j, \operatorname{tang}}\right\|_{L^{2}(S)}^{2}\right)^{2-\frac{p}{2}} M^{\frac{p}{2}-1} \\
& +A_{\alpha}(H)^{2-\frac{p}{2}} R^{2-\frac{p}{2}}\left(\sum_{\tau}\left\|f_{\tau, j, \text { tang }}\right\|_{L^{2}(S)}^{2}\right)^{2-\frac{p}{2}} \mathcal{E}^{\frac{p}{2}-1}
\end{aligned}
$$

(since $\left.\frac{p}{2}-1 \leq 1\right)$. By Lemma 8 -A (applied to a single subset $\mathbb{T}_{j, \text { tang }} \subset \mathbb{T}$ ), we have $\left\|f_{\tau, j, \text { tang }}\right\|_{L^{2}(S)}^{2} \lesssim\left\|f_{\tau}\right\|_{L^{2}(S)}^{2}$, so $\left(\sum_{\tau}\left\|f_{\tau, j, \text { tang }}\right\|_{L^{2}(S)}^{2}\right)^{2-\frac{p}{2}} \lesssim\left(\sum_{\tau}\left\|f_{\tau}\right\|_{L^{2}(S)}^{2}\right)^{2-\frac{p}{2}}$, and so

$$
\begin{aligned}
& A_{\alpha}(H)^{2-\frac{p}{2}} R^{2-\frac{p}{2}}\left(\sum_{\tau}\left\|f_{\tau, j, \text { tang }}\right\|_{L^{2}(S)}^{2}\right)^{2-\frac{p}{2}} \mathcal{E}^{\frac{p}{2}-1} \\
& \quad \lesssim A_{\alpha}(H)^{2-\frac{p}{2}} R^{2-\frac{p}{2}}\left(\sum_{\tau}\left\|f_{\tau}\right\|_{L^{2}(S)}^{2}\right)^{2-\frac{p}{2}}\left(R^{-N+4}\left(\sum_{\tau}\left\|f_{\tau}\right\|_{L^{2}(S)}^{2}\right)^{2}\right)^{\frac{p}{2}-1} \\
& \quad=A_{\alpha}(H)^{2-\frac{p}{2}} R^{1-(N-3)(p-2) / 2}\left(\sum_{\tau}\left\|f_{\tau}\right\|_{L^{2}(S)}^{2}\right)^{\frac{p}{2}} .
\end{aligned}
$$

Thus the error term can be handled with the aid of (25) as in the proof of the previous theorem.

We write the main term as

$$
R^{O(\delta)} A_{\alpha}(H)^{2-\frac{p}{2}} R^{\frac{5}{2}-\frac{3}{4} p}\left(\sum_{\tau}\left\|f_{\tau, j, \operatorname{tang}}\right\|_{L^{2}(S)}^{2}\right)^{2-\frac{p}{2}} M^{\nu} M^{\frac{p}{2}-1-\nu},
$$

where $\nu$ is a positive number that will be determined later. Following $[8$, we will estimate $M$ in two different ways. As we saw during the proof of Theorem 11.1 , part (i) of Proposition 7.2 tells us that

$\sum_{T_{1} \in \mathbb{T}_{j, \text { tang }}}\left\|f_{\tau_{1}, T_{1}}\right\|_{L^{2}(S)}^{2} \lesssim\left\|f_{\tau_{1}}\right\|_{L^{2}(S)}^{2} \quad$ and $\quad \sum_{T_{2} \in \mathbb{T}_{j, \operatorname{tang}}}\left\|f_{\tau_{2}, T_{2}}\right\|_{L^{2}(S)}^{2} \lesssim\left\|f_{\tau_{2}}\right\|_{L^{2}(S)}^{2}$,

so $M \lesssim\left(\sum_{\tau}\left\|f_{\tau}\right\|_{L^{2}(S)}^{2}\right)^{2}$, and so

$$
\left(\sum_{\tau}\left\|f_{\tau, j, \operatorname{tang}}\right\|_{L^{2}(S)}^{2}\right)^{2-\frac{p}{2}} M^{\nu} \lesssim\left(\sum_{\tau}\left\|f_{\tau}\right\|_{L^{2}(S)}^{2}\right)^{2-\frac{p}{2}+2 \nu}
$$


In order to estimate $M^{(p / 2)-1-\nu}$, we use Lemma 8 -D as in the proof of the previous theorem to get $\sum_{T_{1} \in \mathbb{T}_{j, \text { tang }}}\left\|f_{\tau_{1}, T_{1}}\right\|_{L^{2}(S)}^{2} \lesssim R^{O(\delta)} R^{-1 / 2}$ (recall that $b=1$ in this theorem), and likewise with $\tau_{1}, T_{1}$ replaced by $\tau_{2}, T_{2}$. Therefore,

$$
M^{\frac{p}{2}-1-\nu} \lesssim R^{O(\delta)}\left(\frac{1}{R}\right)^{\frac{p}{2}-1-\nu}
$$

provided $p \geq 2(1+\nu)$.

Putting the bounds together, we arrive at

$$
\begin{aligned}
\int_{B_{j} \cap W} & \operatorname{Bil}_{P, \delta} E f_{j, \operatorname{tang}}(x)^{p} H(x) d x \\
\lesssim & R^{O(\delta)} A_{\alpha}(H)^{2-\frac{p}{2}} R^{\frac{5}{2}-\frac{3}{4} p}\left(\sum_{\tau}\left\|f_{\tau}\right\|_{L^{2}(S)}^{2}\right)^{2-\frac{p}{2}+2 \nu}\left(\frac{1}{R}\right)^{\frac{p}{2}-1-\nu} \\
& \quad+R^{1-(N-3)(p-2) / 2} A_{\alpha}(H)^{2-\frac{p}{2}}\left(\sum_{\tau}\left\|f_{\tau}\right\|_{L^{2}(S)}^{2}\right)^{\frac{p}{2}} \\
& \lesssim \quad R^{O(\delta)} A_{\alpha}(H)^{2-\frac{p}{2}} R^{\frac{5}{2}-\frac{3}{4} p}\left(\frac{1}{R}\right)^{\frac{p}{2}-1-\nu}\left(\sum_{\tau}\left\|f_{\tau}\right\|_{L^{2}(S)}^{2}\right)^{2-\frac{p}{2}+2 \nu}
\end{aligned}
$$

provided $p \geq 2(1+\nu)$. Then

We now determine $\nu$. We need to have $2-\frac{p}{2}+2 \nu=\frac{3}{2}+\epsilon$, so $\nu=\frac{p}{4}-\frac{1}{4}+\frac{\epsilon}{2}$. Then

$$
\frac{p}{2}-1-\nu=\frac{p}{4}-\frac{3}{4}-\frac{\epsilon}{2} \geq 0 \Longleftrightarrow p \geq 3+2 \epsilon,
$$

and

$$
R^{\frac{5}{2}-\frac{3}{4} p}\left(\frac{1}{R}\right)^{\frac{p}{2}-1-\nu}=R^{\frac{5}{2}-\frac{3}{4} p}\left(\frac{1}{R}\right)^{\frac{p}{4}-\frac{3}{4}-\frac{\epsilon}{2}}=R^{\epsilon / 2} R^{\frac{13}{4}-p}=R^{\epsilon / 2} .
$$

Thus

$$
\int_{B_{j} \cap W} \operatorname{Bil}_{P, \delta} E f_{j, \text { tang }}(x)^{p} H(x) d x \lesssim R^{O(\delta)} R^{\epsilon / 2} A_{\alpha}(H)^{2-\frac{p}{2}}\left(\sum_{\tau}\left\|f_{\tau}\right\|_{L^{2}(S)}^{2}\right)^{\frac{3}{2}+\epsilon} .
$$

We note that the implicit constant in the $R^{O(\delta)}$ factor does not depend on $b$.

Invoking Theorem 9.1 (with $q_{2}=1 / 2, q_{1}=2-(p / 2)$, and $q_{0}=3 / 4$ ), we conclude that to every sufficiently small $\epsilon$ there are constants $K=K(\epsilon)$ and $C_{\epsilon}$ such that $\lim _{\epsilon \rightarrow 0} K(\epsilon)=\infty$ and

$$
\begin{aligned}
& \int_{B_{R}} \operatorname{Br}_{\beta} E f(x)^{p} H(x) d x \\
& \quad \leq C_{\epsilon} R^{3 \epsilon / 4} A_{\alpha}(H)^{2-(p / 2)}\left(\sum_{\tau}\left\|f_{\tau}\right\|_{L^{2}(S)}^{2}\right)^{(3 / 2)+\epsilon} R^{\delta_{\text {trans }} \log \left(K^{\epsilon} \beta m\right)}
\end{aligned}
$$

for all $\beta \geq K^{-\epsilon}, m \geq 1, R \geq 1$, and $f \in \Lambda(R, K, m, 1)$.

Given a function $f \in L^{2}(S)$ that satisfies

$$
\int_{B\left(\xi_{0}, R^{-1 / 2}\right) \cap S}|f(\xi)|^{2} d \sigma(\xi) \leq \frac{1}{R}
$$

for all $\xi_{0} \in S$, then, writing $f=\sum_{\tau} f_{\tau}$ with $\operatorname{supp} f_{\tau} \subset \tau$ and $\left(\operatorname{supp} f_{\tau}\right) \cap\left(\operatorname{supp} f_{\tau^{\prime}}\right)=$ $\emptyset$ if $\tau \neq \tau^{\prime}$, we see that $f \in \Lambda(R, K, m, 1)$ and $\sum_{\tau}\left\|f_{\tau}\right\|_{L^{2}(S)}^{2}=\|f\|_{L^{2}(S)}^{2}$. Applying the above estimate with $\beta=K^{-\epsilon}$, we obtain the desired result. 


\section{Parabolic Scaling and the main induction ARgument}

Suppose $\tau$ is a cap in $S$ of center $\left(\omega_{0}, h\left(\omega_{0}\right)\right)$ and radius $r \leq 1$. Following 8 , for $\omega \in B^{2}\left(\omega_{0}, r\right)$, we define

$$
\widetilde{h}(\omega)=h(\omega)-h\left(\omega_{0}\right)-\left(\omega-\omega_{0}\right) \cdot \nabla h\left(\omega_{0}\right)
$$

Also, for $|\eta| \leq 1$, we define

$$
h_{1}(\eta)=r^{-2} \widetilde{h}\left(\omega_{0}+r \eta\right)=r^{-2} \widetilde{h}(\omega)
$$

and we let $S_{1}$ be the graph of $h_{1}$ over $B^{2}(0,1)$.

To every function $f$ on $\tau$ we associate a function $g$ on $S_{1}$ defined by

$$
g\left(\eta, h_{1}(\eta)\right)=r^{2} f\left(\omega_{0}+r \eta, h\left(\omega_{0}+r \eta\right)\right) J_{h}\left(\omega_{0}+r \eta\right) J_{h_{1}}(\eta)^{-1},
$$

where $J_{h}=\sqrt{1+|\nabla h|^{2}}$ and $J_{h_{1}}=\sqrt{1+\left|\nabla h_{1}\right|^{2}}$. Then

$$
\begin{aligned}
& \left|\int_{B^{2}\left(\omega_{0}, r\right)} f(\omega, h(\omega)) e^{-2 \pi i\left(\left(x_{1}, x_{2}\right) \cdot \omega+x_{3} h(\omega)\right)} J_{h}(\omega) d \omega\right| \\
& =\mid \int_{B^{2}\left(\omega_{0}, r\right)} f(\omega, h(\omega)) e^{-2 \pi i\left(\left(\left(x_{1}, x_{2}\right)+x_{3} \nabla h\left(\omega_{0}\right)\right) \cdot\left(\omega-\omega_{0}\right)+x_{3} r^{2} h_{1}\left(\left(\omega-\omega_{0}\right) / r\right)\right)} \\
& \quad \times J_{h}(\omega) d \omega \mid \\
& =\mid \int_{B^{2}(0,1)} f\left(\omega_{0}+r \eta, h\left(\omega_{0}+r \eta\right)\right) e^{-2 \pi i\left(\left(\left(x_{1}, x_{2}\right)+x_{3} \nabla h\left(\omega_{0}\right)\right) \cdot(r \eta)+x_{3} r^{2} h_{1}(\eta)\right)} \\
& \quad \times J_{h}\left(\omega_{0}+r \eta\right) r^{2} d \eta \mid \\
& =\left|\int_{B^{2}(0,1)} g\left(\eta, h_{1}(\eta)\right) e^{-2 \pi i\left(\left(\left(r x_{1}, r x_{2}\right)+r x_{3} \nabla h\left(\omega_{0}\right)\right) \cdot \eta+r^{2} x_{3} h_{1}(\eta)\right)} J_{h_{1}}(\eta) d \eta\right|,
\end{aligned}
$$

where we have applied the change of variables $\eta=\left(\omega-\omega_{0}\right) / r$. Thus

$$
\left|E_{S} f(x)\right|=\left|E_{S_{1}} g\left(\left(r x_{1}, r x_{2}\right)+r x_{3} \nabla h\left(\omega_{0}\right), r^{2} x_{3}\right)\right|
$$

for all $x \in \mathbb{R}^{3}$. Also,

$$
\begin{aligned}
& \int_{B^{2}(0,1)}\left|g\left(\eta, h_{1}(\eta)\right)\right|^{2} J_{h_{1}}(\eta) d \eta \\
& \quad=r^{2} \int_{B^{2}(0,1)}\left|f\left(\omega_{0}+r \eta, h\left(\omega_{0}+r \eta\right)\right)\right|^{2} J_{h}\left(\omega_{0}+r \eta\right)^{2} J_{h_{1}}(\eta)^{-1} r^{2} d \eta
\end{aligned}
$$

so applying the change of variables $\omega=\omega_{0}+r \eta$, we see that

$$
\begin{aligned}
& \int_{B^{2}(0,1)}\left|g\left(\eta, h_{1}(\eta)\right)\right|^{2} J_{h_{1}}(\eta) d \eta \\
& \left.\quad=r^{2} \int_{B^{2}\left(\omega_{0}, r\right)}|f(\omega, h(\omega))|^{2} J_{h}(\omega)^{2} J_{h_{1}}\left(\left(\omega-\omega_{0}\right) / r\right)\right)^{-1} d \omega
\end{aligned}
$$

Clearly, $\left.J_{h_{1}}\left(\left(\omega-\omega_{0}\right) / r\right)\right) \geq 1$ and (by (19) $) J_{h}(\omega) \leq 3$ for all $\omega \in B^{2}\left(\omega_{0}, r\right)$, so

$$
\int_{B^{2}(0,1)}\left|g\left(\eta, h_{1}(\eta)\right)\right|^{2} J_{h_{1}}(\eta) d \eta \leq 3 r^{2} \int_{B^{2}\left(\omega_{0}, r\right)}|f(\omega, h(\omega))|^{2} J_{h}(\omega) d \omega .
$$

Define the linear map $T: \mathbb{R}^{3} \rightarrow \mathbb{R}^{3}$ by

$$
T x=\left(\left(r x_{1}, r x_{2}\right)+r x_{3} \nabla h\left(\omega_{0}\right), r^{2} x_{3}\right) .
$$


As we saw above, we have

$$
\left|E_{S} f(x)\right|=\left|E_{S_{1}} g(T x)\right|
$$

so that

$$
\int_{B(0, R)}\left|E_{S} f(x)\right|^{p} H(x) d x=\int_{B(0, R)}\left|E_{S_{1}} g(T x)\right|^{p} H(x) d x .
$$

Applying the change of variables $u=T x$, this becomes

$$
\int_{B(0, R)}\left|E_{S} f(x)\right|^{p} H(x) d x=\int_{T(B(0, R))}\left|E_{S_{1}} g(u)\right|^{p} H\left(T^{-1} u\right) r^{-4} d u .
$$

Since (by (19))

$$
|T x| \leq r\left|\left(x_{1}, x_{2}\right)\right|+r\left|x_{3}\right|\left|\nabla h\left(\omega_{0}\right)\right|+r^{2}\left|x_{3}\right| \leq\left(1+\frac{7}{4}\left|\omega_{0}\right|+r\right) r|x|,
$$

it follows that $T(B(0, R)) \subset B(0,4 r R)$, and hence

$$
\int_{B(0, R)}\left|E_{S} f(x)\right|^{p} H(x) d x \leq \int_{B(0,4 r R)}\left|E_{S_{1}} g(u)\right|^{p} H\left(T^{-1} u\right) r^{-4} d u .
$$

Let $H^{\prime}=H \circ T^{-1}$. We need to study

$$
\int_{B\left(u_{0}, t\right)} H^{\prime}(u) d u=\int_{B\left(u_{0}, t\right)} H\left(T^{-1} u\right) d u
$$

for $u_{0} \in \mathbb{R}^{3}$ and $t \geq 1$. We begin by noticing that

$$
u \in B\left(u_{0}, t\right) \Longleftrightarrow|T v| \leq t \Longleftrightarrow v \cdot A v \leq t^{2},
$$

where $v=T^{-1} u-T^{-1} u_{0}$ and

$$
A=T^{t} T=\left(\begin{array}{ccc}
r^{2} & 0 & r^{2} \partial_{1} h\left(\omega_{0}\right) \\
0 & r^{2} & r^{2} \partial_{2} h\left(\omega_{0}\right) \\
r^{2} \partial_{1} h\left(\omega_{0}\right) & r^{2} \partial_{2} h\left(\omega_{0}\right) & r^{2}\left|\nabla h\left(\omega_{0}\right)\right|^{2}+r^{4}
\end{array}\right) .
$$

The characteristic polynomial of this matrix is

$$
|A-\lambda I|=\left(r^{2}-\lambda\right)\left(\lambda^{2}-\left(r^{2} J_{h}\left(\omega_{0}\right)^{2}+r^{4}\right) \lambda+r^{6}\right) .
$$

Since $J_{h}\left(\omega_{0}\right) \geq 1$, we have

$$
\left(r^{2} J_{h}\left(\omega_{0}\right)^{2}+r^{4}\right)^{2}-4 r^{6} \geq\left(r^{2}+r^{4}\right)^{2}-4 r^{6}=r^{4}+r^{8}-2 r^{6}=\left(r^{2}-r^{4}\right)^{2} \geq 0,
$$

so the eigenvalues of $A$ are

$$
\lambda_{1}=r^{2}, \quad \lambda_{2}=\frac{2 r^{6}}{r^{2} J_{h}\left(\omega_{0}\right)^{2}+r^{4}+\sqrt{\left(r^{2} J_{h}\left(\omega_{0}\right)^{2}+r^{4}\right)^{2}-4 r^{6}}},
$$

and

$$
\lambda_{3}=\frac{r^{2} J_{h}\left(\omega_{0}\right)^{2}+r^{4}+\sqrt{\left(r^{2} J_{h}\left(\omega_{0}\right)^{2}+r^{4}\right)^{2}-4 r^{6}}}{2} .
$$

Since $1 \leq J_{h}\left(\omega_{0}\right) \leq 3$, it follows that

$$
\lambda_{2} \geq \frac{r^{6}}{r^{2} J_{h}\left(\omega_{0}\right)^{2}+r^{4}} \geq \frac{r^{6}}{9 r^{2}+r^{4}} \geq \frac{r^{4}}{10} \quad \text { and } \quad \lambda_{3} \geq \frac{r^{2}}{2} .
$$

Therefore, the image of $B\left(u_{0}, t\right)$ under $T^{-1}$ is contained in an ellipsoid of center $x_{0}=$ $T^{-1} u_{0}$, two short principal axes of length $2 \sqrt{2} t / r$, and long principal axis of length 
$2 \sqrt{10} t / r^{2}$. This ellipsoid can be covered by balls $B\left(x_{1}, 4 t / r\right), \ldots, B\left(x_{N}, 4 t / r\right)$ with $N \leq 3 / r$, so after applying the change of variables $x=T^{-1} u$ we see that

$$
\begin{aligned}
& \int_{B\left(u_{0}, t\right)} H^{\prime}(u) d u=\int_{T^{-1}\left(B\left(u_{0}, t\right)\right)} H(x) r^{4} d x \\
& \leq r^{4} \sum_{j=1}^{N} \int_{B\left(x_{j}, 4 t / r\right)} H(x) d x \leq r^{4} N A_{\alpha}(H)\left(\frac{4 t}{r}\right)^{\alpha} \leq(3)\left(4^{3}\right) r^{3-\alpha} A_{\alpha}(H) t^{\alpha}
\end{aligned}
$$

for all $u_{0} \in \mathbb{R}^{3}$ and $t \geq 1$. Thus

$$
A_{\alpha}\left(H^{\prime}\right) \leq(192) r^{3-\alpha} A_{\alpha}(H) .
$$

Theorem 12.1. Let $0<\alpha \leq 3,3 \leq p \leq 4,2 \leq \gamma \leq 3,0 \leq q_{1} \leq 1, q_{2} \geq 0$, and $c>0$.

Suppose that we have the following estimate on the broad part of Ef: to every $\epsilon \in(0, c)$ there are constants $K(\epsilon)$ and $\bar{C}_{\epsilon}$ such that $\lim _{\epsilon \rightarrow 0} K(\epsilon)=\infty$ and

$$
\int_{B(0, R)} \operatorname{Br}_{K^{-\epsilon}} E f(x)^{p} H(x) d x \leq \bar{C}_{\epsilon} R^{\epsilon} A_{\alpha}(H)^{q_{1}} R^{q_{2}}\|f\|_{L^{2}(S)}^{\gamma}\|f\|_{L^{\infty}(S)}^{p-\gamma}
$$

for all radii $R \geq 1$, weights $H$ of dimension $\alpha$, functions $h$ satisfying conditions (i)-(iv) of Assumption 8.1, and functions $f \in L^{\infty}(S)$.

If $2 p-\alpha-1-\gamma>0$, then there is a constant $c^{\prime}$, which only depends on $\alpha, p$, and $\gamma$, such that for $0<\epsilon<c^{\prime}$ we have

$$
\int_{B(0, R)}|E f(x)|^{p} H(x) d x \leq C_{\epsilon} R^{\epsilon}\left(\max \left[A_{\alpha}(H), A_{\alpha}(H)^{q_{1}}\right]\right) R^{q_{2}}\|f\|_{L^{2}(S)}^{\gamma}\|f\|_{L^{\infty}(S)}^{p-\gamma}
$$

with

$$
C_{\epsilon}=2\left(\bar{C}_{\epsilon}+10^{4} \sigma(S)^{4}\right)
$$

for all radii $R \geq 1$, weights $H$ of dimension $\alpha$, functions $h$ satisfying conditions (i)-(iv) of Assumption 8.1, and functions $f \in L^{\infty}(S)$.

Proof. We are going to prove the theorem by induction on $R$. The estimate is true for $1 \leq R \leq 10$ :

$$
\begin{aligned}
\int_{B(0, R)}|E f(x)|^{p} H(x) d x & \leq\|f\|_{L^{1}(S)}^{p} \int_{B(0,10)} H(x) d x \\
& =\left(\int_{B(0,10)} H(x) d x\right)^{1-q_{1}+q_{1}}\|f\|_{L^{1}(S)}^{p} \\
& \leq R^{\epsilon+q_{2}}|B(0,10)|^{1-q_{1}} A_{\alpha}(H)^{q_{1}}\left(10^{\alpha q_{1}}\right)\|f\|_{L^{1}(S)}^{\gamma}\|f\|_{L^{1}(S)}^{p-\gamma} \\
& \leq\left(5^{1-q_{1}}\right)\left(10^{3}\right) \sigma(S)^{4} R^{\epsilon} A_{\alpha}(H)^{q_{1}} R^{q_{2}}\|f\|_{L^{2}(S)}^{\gamma}\|f\|_{L^{\infty}(S)}^{p-\gamma},
\end{aligned}
$$

where we have used the fact that $\sigma(S) \geq 1$ for all $h$.

Suppose $R \geq 10$ and our estimate is true for all functions $h$ satisfying conditions (i)-(iv) of Assumption 8.1, weights $H$ of dimension $\alpha$, and all radii in the interval 
$[1, R / 2]$. Then

$$
\begin{aligned}
& \int_{B(0, R)}|E f(x)|^{p} H(x) d x \\
& \quad \leq \int_{B(0, R)} \operatorname{Br}_{K^{-\epsilon}} E f(x)^{p} H(x) d x+K^{\epsilon} \sum_{\tau} \int_{B(0, R)}\left|E f_{\tau}(x)\right|^{p} H(x) d x \\
& \quad \leq \bar{C}_{\epsilon} R^{\epsilon} A_{\alpha}(H)^{q_{1}} R^{q_{2}}\|f\|_{L^{2}(S)}^{\gamma}\|f\|_{L^{\infty}(S)}^{p-\gamma}+K^{\epsilon} \sum_{\tau} \int_{B(0, R)}\left|E f_{\tau}(x)\right|^{p} H(x) d x .
\end{aligned}
$$

We have $K$ functions $f_{\tau}$ each supported in a cap of diameter $r=1 / K$, and hence in a set of the form $B\left(\xi_{\tau}, \rho\right) \cap S$ with $r \leq \rho \leq 3 r$ and $\xi_{\tau} \in S$. We are going to use parabolic scaling and the induction hypothesis to bound $\sum_{\tau} \int_{B(0, R)}\left|E f_{\tau}\right|^{p} H(x) d x$. We let $\phi$ be a non-negative Schwartz function on $\mathbb{R}^{3}$ such that $\phi \geq 1$ on the unit ball and $\widehat{\phi}$ is supported in the unit ball, and we observe that

$$
\left|\phi_{\rho}\left(\xi-\xi_{\tau}\right)\right| \geq \frac{1}{\rho^{3}} \quad \text { on } \quad B\left(\xi_{\tau}, \rho\right) .
$$

We also define the function $F_{\tau}$ on $B\left(\xi_{\tau}, \rho\right) \cap S$ by the equation

$$
f_{\tau}(\xi)=\phi_{\rho}\left(\xi-\xi_{\tau}\right) F_{\tau}(\xi)
$$

and we observe that $\left|F_{\tau}\right| \leq \rho^{3}\left|f_{\tau}\right|$. Then

$$
E f_{\tau}(x)=\left(\phi_{\rho}\left(\cdot-\xi_{\tau}\right) \widehat{)} * E F_{\tau}(x)=\int e^{-2 \pi i(x-y) \cdot \xi_{\tau}} \widehat{\phi}(\rho(x-y)) E F_{\tau}(y) d y,\right.
$$

so that

$$
\begin{aligned}
\left|E f_{\tau}(x)\right|^{p} & \leq\left(\int|\widehat{\phi}(\rho(x-y))|\left|E F_{\tau}(y)\right| d y\right)^{p} \\
& \leq\left(\int|\widehat{\phi}(\rho(x-y))| d y\right)^{p-1}\left(\int\left|E F_{\tau}(y)\right|^{p}|\widehat{\phi}(\rho(x-y))| d y\right) \\
& =\frac{1}{\rho^{3(p-1)}}\|\widehat{\phi}\|_{L^{1}}^{p-1} \int\left|E F_{\tau}(y)\right|^{p}|\widehat{\phi}(\rho(x-y))| d y
\end{aligned}
$$

which gives

$$
\begin{aligned}
& \int_{B(0, R)}\left|E f_{\tau}(x)\right|^{p} H(x) d x \\
& \quad \leq \frac{\rho^{3}}{\rho^{3 p}}\|\widehat{\phi}\|_{L^{1}}^{p-1} \int\left|E F_{\tau}(y)\right|^{p} \int_{B(0, R)}|\widehat{\phi}(\rho(x-y))| H(x) d x d y .
\end{aligned}
$$

We now define the function $\mathcal{H}$ on $\mathbb{R}^{3}$ by

$$
\mathcal{H}(y)=\|\widehat{\phi}\|_{L^{\infty}}^{-1} A_{\alpha}(H)^{-1} \rho^{\alpha} \int_{B(0, R)}|\widehat{\phi}(\rho(x-y))| H(x) d x,
$$

notice that $\mathcal{H}$ is supported in the ball $B\left(0, R+\rho^{-1}\right)$, and conclude that

$$
\begin{aligned}
& \int_{B(0, R)}\left|E f_{\tau}(x)\right|^{p} H(x) d x \\
& \quad \leq\|\widehat{\phi}\|_{L^{\infty}} A_{\alpha}(H)\|\widehat{\phi}\|_{L^{1}}^{p-1} \rho^{3-\alpha} \rho^{-3 p} \int_{B\left(0, R+\rho^{-1}\right)}\left|E F_{\tau}(y)\right|^{p} \mathcal{H}(y) d y .
\end{aligned}
$$


Since $\widehat{\phi}$ is supported in the unit ball, we have

$$
\|\widehat{\phi}\|_{L^{1}} \leq|B(0,1)|\|\widehat{\phi}\|_{L^{\infty}} \leq 5\|\widehat{\phi}\|_{L^{\infty}} .
$$

Thus

$$
\begin{aligned}
& \int_{B(0, R)}\left|E f_{\tau}(x)\right|^{p} H(x) d x \\
& \quad \leq 5^{3}\|\widehat{\phi}\|_{L^{\infty}}^{4} A_{\alpha}(H) \rho^{3-\alpha-3 p} \int_{B\left(0, R+\rho^{-1}\right)}\left|E F_{\tau}(y)\right|^{p} \mathcal{H}(y) d y
\end{aligned}
$$

$\left(\|\widehat{\phi}\|_{L^{\infty}} \geq 1\right.$ because $\left.\widehat{\phi}(0)=\|\phi\|_{L^{1}} \geq 1\right)$.

The function $\mathcal{H}$ is a weight on $\mathbb{R}^{3}$ of the same dimension as $H$. In fact,

$$
\mathcal{H}(y) \leq \frac{\rho^{\alpha}\|\widehat{\phi}\|_{L^{\infty}}^{-1}}{A_{\alpha}(H)}\|\widehat{\phi}\|_{L^{\infty}} \int_{B(y, 1 / \rho)} H(x) d x \leq \frac{\rho^{\alpha}}{A_{\alpha}(H)} A_{\alpha}(H)\left(\frac{1}{\rho}\right)^{\alpha}=1
$$

(provided $1 / \rho \geq 1$ ) for all $y \in \mathbb{R}^{3}$, so $\|\mathcal{H}\|_{L^{\infty}} \leq 1$. Also,

$$
\begin{aligned}
\int_{B\left(y_{0}, t\right)} \mathcal{H}(y) d y & =\int \chi_{B\left(y_{0}, t\right)}(y) \mathcal{H}(y) d y \\
& =\frac{\rho^{\alpha}\|\widehat{\phi}\|_{L^{\infty}}^{-1}}{A_{\alpha}(H)} \int_{B(0, R)} \int \chi_{B\left(y_{0}, t\right)}(y)|\widehat{\phi}(\rho(x-y))| d y H(x) d x .
\end{aligned}
$$

Applying the change of variables $v=\rho(x-y)$ to the inner integral, we get

$$
\begin{aligned}
\int_{B\left(y_{0}, t\right)} \mathcal{H}(y) d y & =\frac{\rho^{\alpha-3}\|\widehat{\phi}\|_{L^{\infty}}^{-1}}{A_{\alpha}(H)} \int_{B(0, R)} \int \chi_{B\left(y_{0}, t\right)}\left(x-\rho^{-1} v\right)|\widehat{\phi}(v)| d v H(x) d x \\
& =\frac{\rho^{\alpha-3}\|\widehat{\phi}\|_{L^{\infty}}^{-1}}{A_{\alpha}(H)} \int_{B(0, R)}|\widehat{\phi}(v)| \int \chi_{B\left(y_{0}, t\right)}\left(x-\rho^{-1} v\right) H(x) d x d v \\
& =\frac{\rho^{\alpha-3}\|\widehat{\phi}\|_{L^{\infty}}^{-1}}{A_{\alpha}(H)} \int_{B(0, R)}|\widehat{\phi}(v)| \int \chi_{B\left(y_{0}+\rho^{-1} v, t\right)}(x) H(x) d x d v \\
& \leq \frac{\rho^{\alpha-3}\|\widehat{\phi}\|_{L^{\infty}}^{-1}}{A_{\alpha}(H)} \int_{B(0, R)}|\widehat{\phi}(v)| A_{\alpha}(H) t^{\alpha} d v \\
& \leq\|\widehat{\phi}\|_{L^{\infty}}^{-1}\|\widehat{\phi}\|_{L^{1}} \rho^{\alpha-3} t^{\alpha}
\end{aligned}
$$

for all $y_{0} \in \mathbb{R}^{3}$ and $t \geq 1$, so that

$$
A_{\alpha}(\mathcal{H}) \leq\|\widehat{\phi}\|_{L^{\infty}}^{-1}\|\widehat{\phi}\|_{L^{1}} \rho^{\alpha-3} \leq 5 \rho^{\alpha-3} .
$$

We know that $\tau$ is the graph of $h$ over $B^{2}\left(\omega_{0}, r\right)$, so, by (33),

$$
\begin{aligned}
\int_{B\left(0, R+\rho^{-1}\right)}\left|E F_{\tau}(x)\right|^{p} \mathcal{H}(x) d x & =\int_{B\left(0, R+\rho^{-1}\right)}\left|E_{S} F_{\tau}(x)\right|^{p} \mathcal{H}(x) d x \\
& \leq r^{-4} \int_{B(0,4 r R+4)}\left|E_{S_{1}} G(u)\right|^{p} \mathcal{H}^{\prime}(u) d u
\end{aligned}
$$


$\left(B\left(0,4 r R+4 r \rho^{-1}\right) \subset B(0,4 r R+4)\right.$ because $\left.r \leq \rho\right)$, and so (choosing $K$ large enough for $4 r R+4<R / 2$ ) the induction hypothesis tells us that

$$
\begin{aligned}
& \int_{B\left(0, R+\rho^{-1}\right)}\left|E F_{\tau}(x)\right|^{p} \mathcal{H}(x) d x \\
& \quad \leq r^{-4} C_{\epsilon}(4 r R+4)^{\epsilon}\left(\max \left[A_{\alpha}\left(\mathcal{H}^{\prime}\right), A_{\alpha}\left(\mathcal{H}^{\prime}\right)^{q_{1}}\right]\right)(4 r R+4)^{q_{2}}\|G\|_{L^{2}\left(S_{1}\right)}^{\gamma}\|G\|_{L^{\infty}\left(S_{1}\right)}^{p-\gamma} .
\end{aligned}
$$

Since

$$
G\left(\eta, h_{1}(\eta)\right)=r^{2} F_{\tau}\left(\omega_{0}+r \eta, h\left(\omega_{0}+r \eta\right)\right) J_{h}\left(\omega_{0}+r \eta\right) J_{h_{1}}(\eta)^{-1}
$$

and $1 \leq J \leq 3$, it follows that

$$
\|G\|_{L^{\infty}\left(S_{1}\right)} \leq 3 r^{2}\left\|F_{\tau}\right\|_{L^{\infty}(S)} .
$$

Also, since

$$
\int_{B^{2}(0,1)}\left|G\left(\eta, h_{1}(\eta)\right)\right|^{2} J_{h_{1}}(\eta) d \eta \leq 3 r^{2} \int_{B^{2}\left(\omega_{0}, r\right)}\left|F_{\tau}(\omega, h(\omega))\right|^{2} J_{h}(\omega) d \omega,
$$

we have

Therefore,

$$
\|G\|_{L^{2}\left(S_{1}\right)}^{2} \leq 3 r^{2}\left\|F_{\tau}\right\|_{L^{2}(S)}^{2} .
$$

$$
\begin{aligned}
& \int_{B\left(0, R+\rho^{-1}\right)}\left|E F_{\tau}(x)\right|^{p} \mathcal{H}(x) d x \\
& \quad \leq r^{-4} C_{\epsilon}\left(\frac{R}{2}\right)^{\epsilon}\left(\max \left[A_{\alpha}\left(\mathcal{H}^{\prime}\right), A_{\alpha}\left(\mathcal{H}^{\prime}\right)^{q_{1}}\right]\right)\left(\frac{R}{2}\right)^{q_{2}}\|G\|_{L^{2}\left(S_{1}\right)}^{\gamma}\|G\|_{L^{\infty}\left(S_{1}\right)}^{p-\gamma} \\
& \quad \leq 3^{4} r^{2 p-4-\gamma} C_{\epsilon} R^{\epsilon}\left(\max \left[A_{\alpha}\left(\mathcal{H}^{\prime}\right), A_{\alpha}\left(\mathcal{H}^{\prime}\right)^{q_{1}}\right]\right) R^{q_{2}}\left\|F_{\tau}\right\|_{L^{2}(S)}^{\gamma}\left\|F_{\tau}\right\|_{L^{\infty}(S)}^{p-\gamma} .
\end{aligned}
$$

Since (by (34) and (36))

$$
A_{\alpha}\left(\mathcal{H}^{\prime}\right) \leq(192) r^{3-\alpha} A_{\alpha}(\mathcal{H}) \leq(192) r^{3-\alpha}\left(5 \rho^{\alpha-3}\right) \leq 960
$$

and $0 \leq q_{1} \leq 1$, it follows that

$$
\max \left[A_{\alpha}\left(\mathcal{H}^{\prime}\right), A_{\alpha}\left(\mathcal{H}^{\prime}\right)^{q_{1}}\right] \leq 960 .
$$

So

$$
\begin{aligned}
& \int_{B\left(0, R+\rho^{-1}\right)}\left|E F_{\tau}(x)\right|^{p} \mathcal{H}(x) d x \\
& \quad \leq\left(3^{4} \times 960\right) r^{2 p-4-\gamma} C_{\epsilon} R^{\epsilon} R^{q_{2}}\left\|F_{\tau}\right\|_{L^{2}(S)}^{\gamma}\left\|F_{\tau}\right\|_{L^{\infty}(S)}^{p-\gamma},
\end{aligned}
$$

and so (using (35))

$$
\begin{aligned}
& \int_{B(0, R)}\left|E f_{\tau}(x)\right|^{p} H(x) d x \\
& \quad \leq 10^{7}\|\widehat{\phi}\|_{L^{\infty}}^{4} \rho^{3-\alpha-3 p} r^{2 p-4-\gamma} C_{\epsilon} R^{\epsilon} A_{\alpha}(H) R^{q_{2}}\left\|F_{\tau}\right\|_{L^{2}(S)}^{\gamma}\left\|F_{\tau}\right\|_{L^{\infty}(S)}^{p-\gamma} .
\end{aligned}
$$

Recalling that $\left|F_{\tau}\right| \leq \rho^{3}\left|f_{\tau}\right|$, this becomes

$$
\begin{aligned}
& \int_{B(0, R)}\left|E f_{\tau}(x)\right|^{p} H(x) d x \\
& \quad \leq 10^{7}\|\widehat{\phi}\|_{L^{\infty}}^{4} \rho^{3-\alpha} r^{2 p-4-\gamma} C_{\epsilon} R^{\epsilon} A_{\alpha}(H) R^{q_{2}}\left\|f_{\tau}\right\|_{L^{2}(S)}^{\gamma}\left\|f_{\tau}\right\|_{L^{\infty}(S)}^{p-\gamma} \\
& \quad \leq\left(10^{7} \times 3^{3}\right)\|\hat{\phi}\|_{L^{\infty}}^{4} r^{2 p-\alpha-1-\gamma} C_{\epsilon} R^{\epsilon} A_{\alpha}(H) R^{q_{2}}\left\|f_{\tau}\right\|_{L^{2}(S)}^{\gamma}\|f\|_{L^{\infty}(S)}^{p-\gamma}
\end{aligned}
$$


(recall that $\rho \leq 3 r$ ). Thus

$$
\begin{aligned}
& K^{\epsilon} \sum_{\tau} \int_{B(0, R)}\left|E f_{\tau}(x)\right|^{p} H(x) d x \\
& \quad \leq 10^{9}\|\widehat{\phi}\|_{L^{\infty}}^{4} r^{2 p-\alpha-1-\gamma-\epsilon} C_{\epsilon} R^{\epsilon} A_{\alpha}(H) R^{q_{2}}\|f\|_{L^{\infty}(S)}^{p-\gamma} \sum_{\tau}\left\|f_{\tau}\right\|_{L^{2}(S)}^{\gamma} .
\end{aligned}
$$

Now

$$
\sum_{\tau}\left\|f_{\tau}\right\|_{L^{2}(S)}^{\gamma}=\sum_{\tau}\left(\int\left|f_{\tau}\right|^{2} d \sigma\right)^{\gamma / 2} \leq\left(\sum_{\tau} \int\left|f_{\tau}\right|^{2} d \sigma\right)^{\gamma / 2}=\|f\|_{L^{2}(S)}^{\gamma}
$$

provided $\gamma \geq 2$, and

$$
10^{9}\|\widehat{\phi}\|_{L^{\infty}}^{4} r^{2 p-\alpha-1-\gamma-\epsilon} C_{\epsilon} \leq \bar{C}_{\epsilon}+10^{4} \sigma(S)^{4}
$$

provided

so

$$
10^{9}\|\widehat{\phi}\|_{L^{\infty}}^{4} r^{2 p-\alpha-1-\gamma-\epsilon} \leq \frac{1}{2}
$$

$$
\begin{aligned}
& K^{\epsilon} \sum_{\tau} \int_{B_{R}(0)}\left|E f_{\tau}(x)\right|^{p} H(x) d x \\
& \quad \leq \quad\left(\bar{C}_{\epsilon}+10^{4} \sigma(S)^{4}\right) R^{\epsilon} A_{\alpha}(H) R^{q_{2}}\|f\|_{L^{2}(S)}^{\gamma}\|f\|_{L^{\infty}(S)}^{p-\gamma}
\end{aligned}
$$

provided

$$
r^{2 p-\alpha-1-\gamma-\epsilon} \leq 10^{-10}\|\widehat{\phi}\|_{L^{\infty}}^{-4}
$$

Since $\lim _{\epsilon \rightarrow 0} K(\epsilon)=\infty$, the induction closes if $2 p-\alpha-1-\gamma>0$, and we obtain

$$
\int_{B(0, R)}|E f(x)|^{p} H(x) d x \leq C_{\epsilon} R^{\epsilon}\left(\max \left[A_{\alpha}(H), A_{\alpha}(H)^{q_{1}}\right]\right) R^{q_{2}}\|f\|_{L^{2}(S)}^{\gamma}\|f\|_{L^{\infty}(S)}^{p-\gamma},
$$

as desired.

\section{Proof of Theorem 1.1}

(i) We let $b=1$ in Theorem 11.1 Then $p=2(4 \alpha+3) /(2 \alpha+3)$, and to every $0<\epsilon \leq \min \left[c_{0},(p-3) / 2\right]$ there are constants $K=K(\epsilon)$ and $C_{\epsilon}$ such that $\lim _{\epsilon \rightarrow 0} K=\infty$ and

$$
\int_{B(0, R)} \operatorname{Br}_{K^{-\epsilon}} E f(x)^{p} H(x) d x \leq C_{\epsilon} R^{\epsilon} A_{\alpha}(H)^{1-\frac{p}{4}}\|f\|_{L^{2}(S)}^{3+2 \epsilon}
$$

for all functions $f \in L^{2}(S)$ that satisfy the inequality

$$
\int_{B\left(\xi_{0}, R^{-1 / 2}\right) \cap S}|f|^{2} d \sigma \leq \frac{1}{R}
$$

for all $\xi_{0} \in S$.

Given a non-zero function $f \in L^{\infty}(S)$, we see that the function $\|f\|_{L^{\infty}(S)}^{-1} f$ satisfies (37), and the above estimate becomes

$$
\int_{B(0, R)} \operatorname{Br}_{K^{-\epsilon}} E f(x)^{p} H(x) d x \leq \bar{C}_{\epsilon} R^{\epsilon} A_{\alpha}(H)^{1-\frac{p}{4}}\|f\|_{L^{2}(S)}^{3}\|f\|_{L^{\infty}(S)}^{p-3} .
$$

Applying Theorem 12.1 with $q_{1}=1-(p / 4), q_{2}=0$, and $\gamma=3$, we get the required result provided $2 p>\alpha+4$. Solving this inequality for $\alpha$, we get $3 / 2<\alpha<5 / 2$. We have thus proved part (i) except for the case $\alpha=3 / 2$. 
We remind the reader about what we mentioned in $\S 1.1$ concerning the case $\alpha=3 / 2$ of Theorem 1.1. When $\alpha=3 / 2$, parts (i) and (ii) of Theorem 1.1 agree, but the proof belongs to part (ii).

(ii) We suppose first that $\alpha>3 / 2$. We let

$$
b=b_{\epsilon}=\frac{\alpha-(3 / 2)}{2 \epsilon}-\alpha-\frac{1}{2},
$$

in Theorem 11.1. This requires some explanation. The conclusion of Theorem 11.1 holds for $0<\epsilon \leq \min \left[c_{0},(p-3) / 2\right]$. Since $b \geq 1$, we have

$$
\frac{p-3}{2}=\frac{1}{2}\left(\frac{8 \alpha+6 b}{2 \alpha+2 b+1}-3\right) \leq \frac{\alpha-(3 / 2)}{2 \alpha+3} .
$$

So we assume that $c_{0} \leq(\alpha-(3 / 2)) /(2 \alpha+3)$ and choose $b$ to satisfy $\epsilon=(p-3) / 2$. Solving this equation for $b$, we arrive at the solution $b=b_{\epsilon}$ as above.

We, therefore, have the following estimate on the broad part of $E f$ :

$$
\int_{B(0, R)} \operatorname{Br}_{K^{-\epsilon}} E f(x)^{p} H(x) d x \leq C_{\epsilon} R^{(b+1) \epsilon / 2} A_{\alpha}(H)^{1-\frac{p}{4}}\|f\|_{L^{2}(S)}^{3+2 \epsilon}
$$

whenever $R \geq 1, f \in L^{2}(S)$, and

$$
\int_{B\left(\xi_{0}, R^{-1 / 2}\right) \cap S}|f|^{2} d \sigma \leq \frac{1}{R^{(b+1) / 2}}
$$

for all $\xi_{0} \in S$.

Given a non-zero function $f \in L^{2}(S)$, we see that the function $R^{-(b+1) / 4}\|f\|_{L^{2}(S)}^{-1} f$ satisfies (38), and the above estimate becomes

$$
\int_{B(0, R)} \operatorname{Br}_{K^{-\epsilon}} E f(x)^{p} H(x) d x \leq C_{\epsilon} A_{\alpha}(H)^{1-\frac{p}{4}} R^{(p-3)(b+1) / 4}\|f\|_{L^{2}(S)}^{p} .
$$

By Hölder's inequality, we have

$$
\int_{B(0, R)} \operatorname{Br}_{K^{-\epsilon}} E f(x)^{3} H(x) d x \leq\left(A_{\alpha}(H) R^{\alpha}\right)^{1-\frac{3}{p}}\left(\int_{B_{R}(0)} \operatorname{Br}_{K^{-\epsilon}} E f(x)^{p} H(x) d x\right)^{\frac{3}{p}},
$$

and hence

$$
\left(\int_{B(0, R)} \operatorname{Br}_{K^{-\epsilon}} E f(x)^{3} H(x) d x\right)^{\frac{1}{3}} \leq C_{\epsilon}^{\frac{1}{p}} R^{\alpha \frac{p-3}{3 p}} A_{\alpha}(H)^{\frac{1}{12}} R^{\frac{(p-3)(b+1)}{4 p}}\|f\|_{L^{2}(S)} .
$$

Inserting for $b$ its value in term of $\epsilon$, we get

$$
(p-3)(b+1)=\alpha-\frac{3}{2}-\left(\alpha-\frac{1}{2}\right)(2 \epsilon)
$$

so that

$$
\begin{aligned}
\alpha \frac{p-3}{3 p}+\frac{(p-3)(b+1)}{4 p} & =\alpha \frac{2 \epsilon}{3 p}-\left(\alpha-\frac{1}{2}\right) \frac{\epsilon}{2 p}+\frac{1}{4 p}\left(\alpha-\frac{3}{2}\right) \\
& =\frac{\epsilon}{4 p}+\alpha \frac{\epsilon}{6 p}+\frac{1}{4 p}\left(\alpha-\frac{3}{2}\right) \\
& \leq \frac{\epsilon}{4 p}+\frac{\epsilon}{2 p}+\frac{1}{12}\left(\alpha-\frac{3}{2}\right)
\end{aligned}
$$

(because $\alpha \leq 3 \leq p$ ), and hence

$$
\int_{B(0, R)} \operatorname{Br}_{K^{-\epsilon}} E f(x)^{3} H(x) d x \leq \bar{C}_{\epsilon} R^{\epsilon} A_{\alpha}(H)^{\frac{1}{4}} R^{\frac{1}{4}\left(\alpha-\frac{3}{2}\right)}\|f\|_{L^{2}(S)}^{3} .
$$


Applying Theorem 12.1 with $q_{1}=1 / 4, q_{2}=(1 / 4)(\alpha-(3 / 2)), p=3$, and $\gamma=3$, we arrive at the desired result provided $2 p>\alpha+4$, i.e. provided $\alpha<2$.

We have proved part (ii) of Theorem 1.1 in the regime $3 / 2<\alpha<2$ :

$$
\int_{B(0, R)}|E f(x)|^{3} H(x) d x \leq C_{\epsilon}(\alpha, S) R^{\epsilon} A_{\alpha, 3}(H) R^{\frac{1}{4}\left(\alpha-\frac{3}{2}\right)}\|f\|_{L^{2}(S)}^{3}
$$

for all $f \in L^{2}(S)$ and $R \geq 1$. In particular, when $\alpha=(3 / 2)+\epsilon$, this becomes

$$
\int_{B(0, R)}|E f(x)|^{3} H(x) d x \leq C_{\epsilon}(S) R^{\epsilon} A_{(3 / 2)+\epsilon, 3}(H) R^{\epsilon / 4}\|f\|_{L^{2}(S)}^{3} .
$$

But from the definition of $A_{\alpha}(H)$, we see that $A_{\beta}(H) \leq A_{\alpha}(H)$ if $\beta \geq \alpha$, so the same is true for $A_{\alpha, p}(H)$, and so

$$
\int_{B(0, R)}|E f(x)|^{3} H(x) d x \leq C_{\epsilon}(S) R^{2 \epsilon} A_{(3 / 2), 3}(H)\|f\|_{L^{2}(S)}^{3} .
$$

(iii) In this part we use Theorem 11.2, We have the following estimate on the broad part of $E f$ :

$$
\int_{B(0, R)} \operatorname{Br}_{K^{-\epsilon}} E f(x)^{p} H(x) d x \leq C_{\epsilon} R^{\epsilon} A_{\alpha}(H)^{2-\frac{p}{2}}\|f\|_{L^{2}(S)}^{3}\|f\|_{L^{\infty}(S)}^{p-3}
$$

for all $f \in L^{\infty}(S)$, where $p=13 / 4$. Of course,

$$
\|f\|_{L^{2}(S)}^{3}=\|f\|_{L^{2}(S)}^{\gamma}\|f\|_{L^{2}(S)}^{3-\gamma} \lesssim\|f\|_{L^{2}(S)}^{\gamma}\|f\|_{L^{\infty}(S)}^{3-\gamma}
$$

whenever $0 \leq \gamma \leq 3$, so the above estimate implies that

$$
\int_{B(0, R)} \operatorname{Br}_{K^{-\epsilon}} E f(x)^{p} H(x) d x \leq \bar{C}_{\epsilon} R^{\epsilon} A_{\alpha}(H)^{2-\frac{p}{2}}\|f\|_{L^{2}(S)}^{\gamma}\|f\|_{L^{\infty}(S)}^{p-\gamma} .
$$

Applying Theorem 12.1 with $q_{1}=2-(p / 2), q_{2}=0$, and $2 \leq \gamma \leq 3$, we arrive at the desired conclusion provided $2 p-\alpha-1-\gamma>0$, i.e. provided $\gamma<(11 / 2)-\alpha$.

\section{REFERENCES}

[1] J. Bennett and A. Vargas, Randomised circular means of Fourier transforms of measures, Proc. Amer. Math. Soc. 131 (2003), 117-127.

[2] J. Bourgain, Besicovitch type maximal operators and applications to Fourier analysis, Geom. Funct. Anal. 1 (1991), 147-187.

[3] J. Bourgain, Hausdorff dimension and distance sets, Israel J. Math. 87 (1994), 193-201.

[4] J. Bourgain And L. Guth, Bounds on oscillatory integral operators based on multilinear estimates, Geom. Funct. Anal. 21 (2011), 1239-1295.

[5] M. B. ERdoĞAn, A bilinear Fourier extension theorem and applications to the distance set problem, Int. Math. Res. Not. 23 (2005), 1411-1425.

[6] K. J. FAlCONER, On the Hausdorff dimensions of distance sets, Mathematika 32 (1985), $206-212$.

[7] L. Guth, Distinct distance estimates and low degree polynomial partitioning, Discrete Comput. Geom. 53 (2015), 428-444.

[8] L. Guth, A restriction estimate using polynomial partitioning, J. Amer. Math. Soc. 29 (2016), $371-413$.

[9] L. Guth, Restriction estimates using polynomial partitioning II, arXiv:1603.04250.

[10] L. Guth And N. H. Katz, On the Erdös distinct distances problem in the plane, Ann. of Math. (2) 181 (2015), 155-190.

[11] R. LuCÀ And K. Rogers, Average decay of the Fourier transform of measures with applications, arXiv:1503.00105.

[12] P. MatTiLA, Spherical averages of Fourier transforms of measures with finite energy: dimensions of intersections and distance sets, Mathematika 34 (1987), 207-228. 
[13] J. Milnor, On the Betti numbers of real varieties, Proc. Amer. Math. Soc. 15 (1964), 275280.

[14] P. SJöLIN, Estimates of spherical averages of Fourier transforms and dimensions of sets, Mathematika 40 (1993), 322-330.

[15] T. TAO, The Bochner-Riesz conjecture implies the restriction conjecture, Duke Math. J. 96 (1999), 363-375.

[16] T. TAO, A sharp bilinear restrictions estimate for paraboloids, Geom. Funct. Anal. 13 (2003), 1359-1384.

[17] T. TAO, Some recent progress on the restriction conjecture, Fourier analysis and convexity, 217-243, Appl. Numer. Harmon. Anal., Birkhäuser Boston, Boston, MA, 2004.

[18] T. TaO, A. Vargas, and L. Vega, A bilinear approach to the restriction and Kakeya conjectures, J. Amer. Math. Soc. 11 (1998), 967-1000.

[19] T. WolfF, Decay of circular means of Fourier transforms of measures, Int. Math. Res. Not. 10 (1999), 547-567.

[20] T. WolfF, A sharp bilinear cone restriction estimate, Annals of Math. 153 (2001), 661-698.

[21] T. WolfF, Lectures on harmonic analysis, With a foreword by Charles Fefferman and preface by Izabella Łaba, University Lecture Series 29 (eds Laba and Carol Shubin; American Mathematical Society, Providence, RI, 2003).

Department of Mathematics, American University of Beirut, Beirut, Lebanon

E-mail address: bshayya@aub.edu.lb 